\title{
The Science of Sungrazers, Sunskirters, and Other Near-Sun Comets
}

\author{
Geraint H. Jones ${ }^{1,2}$ • Matthew M. Knight ${ }^{3,4} \cdot$ Karl Battams $^{5}$ - Daniel C. Boice ${ }^{6,7,8}$. \\ John Brown ${ }^{9}$ Silvio Giordano ${ }^{10}$ • John Raymond ${ }^{11}$ - Colin Snodgrass ${ }^{12,13}$. \\ Jordan K. Steckloff ${ }^{14,15,16}$ • Paul Weissman ${ }^{14}$ - Alan Fitzsimmons ${ }^{17}$ - Carey Lisse $^{18}$. \\ Cyrielle Opitom ${ }^{19,20}$ - Kimberley S. Birkett ${ }^{1,2,21} \cdot$ Maciej Bzowski $^{22}$ - Alice Decock ${ }^{19,23}$. \\ Ingrid Mann $^{24,25}$ • Yudish Ramanjooloo ${ }^{1,2,26} \cdot$ Patrick McCauley $^{11}$
}

Received: 1 March 2017 / Accepted: 15 November 2017 / Published online: 18 December 2017

(C) The Author(s) 2017. This article is published with open access at Springerlink.com

\begin{abstract}
This review addresses our current understanding of comets that venture close to the Sun, and are hence exposed to much more extreme conditions than comets that are typically studied from Earth. The extreme solar heating and plasma environments that these objects encounter change many aspects of their behaviour, thus yielding valuable information on both the comets themselves that complements other data we have on primitive solar system bodies, as well as on the near-solar environment which they traverse. We propose clear definitions for these comets: We use the term near-Sun comets to encompass all ob-
\end{abstract}

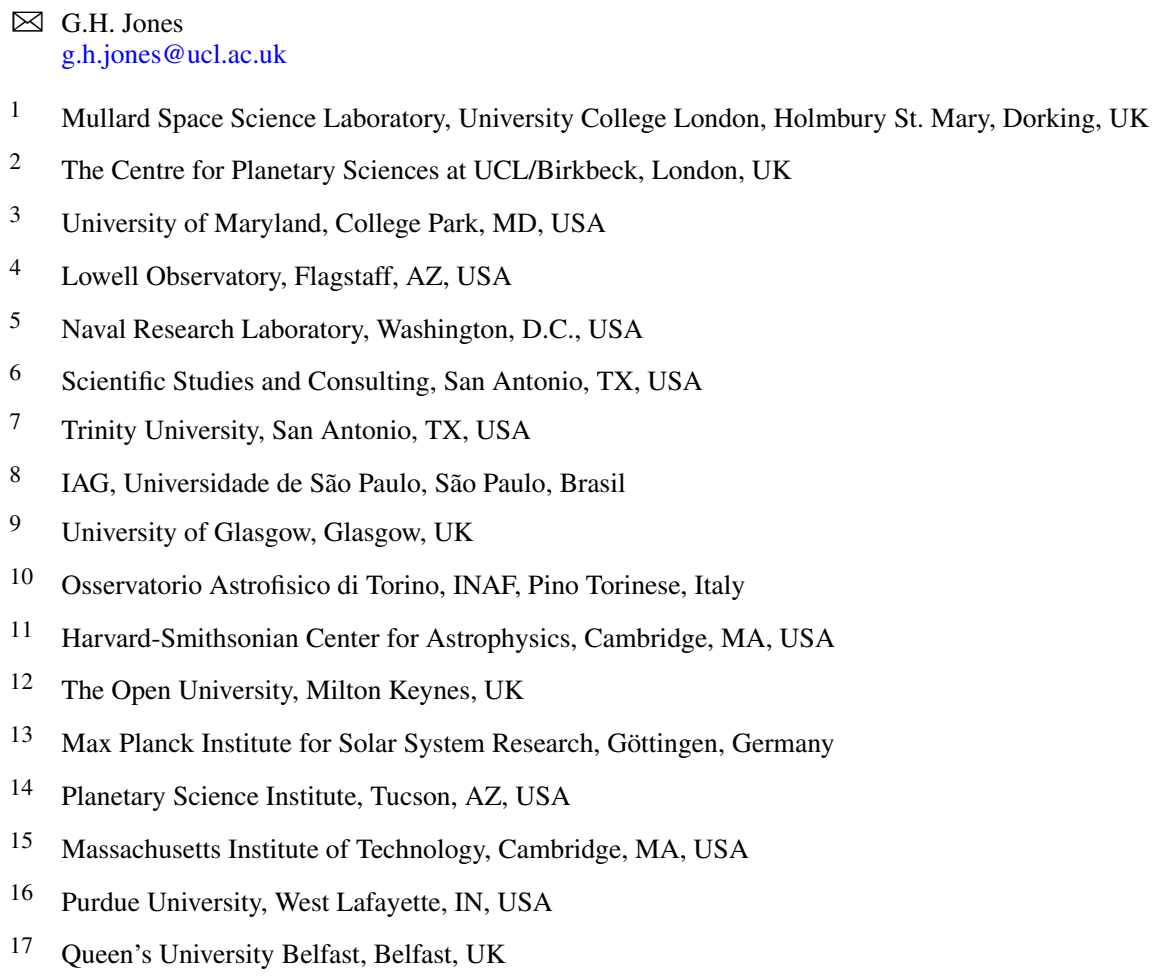


jects that pass sunward of the perihelion distance of planet Mercury (0.307 AU). Sunskirters are defined as objects that pass within 33 solar radii of the Sun's centre, equal to half of Mercury's perihelion distance, and the commonly-used phrase sungrazers to be objects that reach perihelion within 3.45 solar radii, i.e. the fluid Roche limit. Finally, comets with orbits that intersect the solar photosphere are termed sundivers. We summarize past studies of these objects, as well as the instruments and facilities used to study them, including space-based platforms that have led to a recent revolution in the quantity and quality of relevant observations. Relevant comet populations are described, including the Kreutz, Marsden, Kracht, and Meyer groups, near-Sun asteroids, and a brief discussion of their origins. The importance of light curves and the clues they provide on cometary composition are emphasized, together with what information has been gleaned about nucleus parameters, including the sizes and masses of objects and their families, and their tensile strengths. The physical processes occurring at these objects are considered in some detail, including the disruption of nuclei, sublimation, and ionisation, and we consider the mass, momentum, and energy loss of comets in the corona and those that venture to lower altitudes. The different components of comae and tails are described, including dust, neutral and ionised gases, their chemical reactions, and their contributions to the near-Sun environment. Comet-solar wind interactions are discussed, including the use of comets as probes of solar wind and coronal conditions in their vicinities. We address the relevance of work on comets near the Sun to similar objects orbiting other stars, and conclude with a discussion of future directions for the field and the planned ground- and space-based facilities that will allow us to address those science topics.

Keywords Comets $\cdot$ Solar corona $\cdot$ Solar wind

\section{Introduction}

\subsection{Overview}

Comets are primitive aggregates of volatile ices, organics and refractory material condensed from the proto-planetary accretion disk around the Sun. They formed at low temperature, $\sim 20-40 \mathrm{~K}$, during its proto-stellar and young stellar object phases of evolution. Typically $0.3-25 \mathrm{~km}$ in radius, comets are composed of a mixture of ice and organic and silicate material. They likely formed, or started to form, before the planets (Davidsson et al. 2016).

Near-Sun comets were likely relatively common during two periods in the very early solar system. The first was when the nascent Sun and planets grew, and the second during

18 Johns Hopkins University Applied Physics Laboratory, Laurel, USA

University of Liège, Liège, Belgium

European Southern Observatory, Santiago de Chile, Chile

Surrey Satellite Technology Ltd, Guildford, UK

CBK, Warsaw, Poland

Observatoire de Paris, Paris, France

EISCAT Scientific Association, Kiruna, Sweden

UiT The Arctic University of Norway, Troms $\varnothing$, Norway 
Table 1 Proposed formal definitions of Near-Sun Comet types by perihelion distance, $q$. One solar radius, $\mathrm{R}_{\odot}=695700 \mathrm{~km}$. One astronomical unit, $\mathrm{AU}=149.598 \times 10^{6} \mathrm{~km}$

\begin{tabular}{llll}
\hline Comet classification & $q\left(\mathrm{R}_{\odot}\right)$ & $q\left(\times 10^{6} \mathrm{~km}\right)$ & $q(\mathrm{AU})$ \\
\hline Near-Sun & $<66.1$ & $<46.001$ & $<0.307$ \\
Sunskirting & $3.45-33.1$ & $2.393-23.001$ & $0.016-0.1537$ \\
Sungrazing & $1.0-3.45$ & $0.696-2.393$ & $0.0046-0.016$ \\
Sundiving & $<1.0$ & $<0.696$ & $<0.0046$ \\
\hline
\end{tabular}

the planetary migration period that scattered most of the objects out of the Scattered Disk, Kuiper Belt and giant planet region both outwards and inwards towards the Sun (e.g., the "Nice Model" introduced by Gomes et al. 2005). Both the distant Oort Cloud and the closer Kuiper Belt and Scattered Disk likely contribute to the depleted near-Sun comet population observed today.

Observational constraints on Earth-based and most astronomical satellite observatories, mean that our knowledge of most comets is primarily based on observations of these bodies when they are outside the orbit of Venus, i.e., observable in the night sky. However, nearSun comets reach perihelion closer to the Sun than this, experiencing extreme solar wind and insolation conditions, where they undergo thermal desorption (e.g., Martín-Doménech et al. 2014), sublimation, radiation spallation, and other processes. Those comets that approach nearest to our star also experience strong gravitational tides. The latter, along with heat-induced interior stresses, sublimative loss, torqueing, and rotationally induced interior stresses (e.g., Hirabayashi et al. 2016) can lead to the complete destruction of the cometary nucleus. Observing comets close to the Sun is extremely challenging by traditional means, but the data from current solar missions offer new insights into comets in the extreme inner heliosphere. Such comet observations, including their spectra, temporal behaviour, and morphology, reveal valuable information both about the inner heliosphere and about the internal structure and composition of cometary nuclei that is complementary to that obtained from comets observed under more benign conditions. In addition, the frequent complete destruction of many such objects has the potential to reveal the bulk chemical abundances of the whole nucleus rather than merely of surface layers. Coronagraphs and heliospheric imagers record only the optical scattering in the coma and dust tail and the line-of-sight integrated density of electrons in the ion tail, plus some plasma emission lines. They can also reveal important signatures of the solar wind-comet interaction including the local magnetic field directions, the dynamics of the surrounding solar wind, and the time variability of gas and dust evolution.

The presence of these objects may also have a significant effect on the heliosphere itself: dust and gas released near the Sun seed the interplanetary dust population and may be the origin of at least some portion of the "inner source" pickup ions observed in the solar wind farther from the Sun (Bzowski and Królikowska 2005). The value of understanding these objects is clear.

In this work, we propose formal definitions for sub-classes of Near-Sun Comets (Table 1; discussed in detail in Sect. 1.3). Although sungrazing comets constitute the largest class of known comets ( $>50 \%$ of all catalogued comets by number), and include some spectacular objects such as C/1843 D1-The Great Southern Comet of 1843 (Fig. 1)-what are being seen are primarily small fragments of larger original objects. In the case of the group of Kreutz sungrazers, the $>2,900$ catalogued members of the population (Battams and Knight 
Fig. 1 The Great Comet of 1843 in daylight next to the Sun, painted by Charles Piazzi Smyth. Smyth recorded the comet's appearance at the Royal Observatory, Cape of Good Hope, South Africa, during 1843 March 3-6 (@) National Maritime Museum, Greenwich, London)

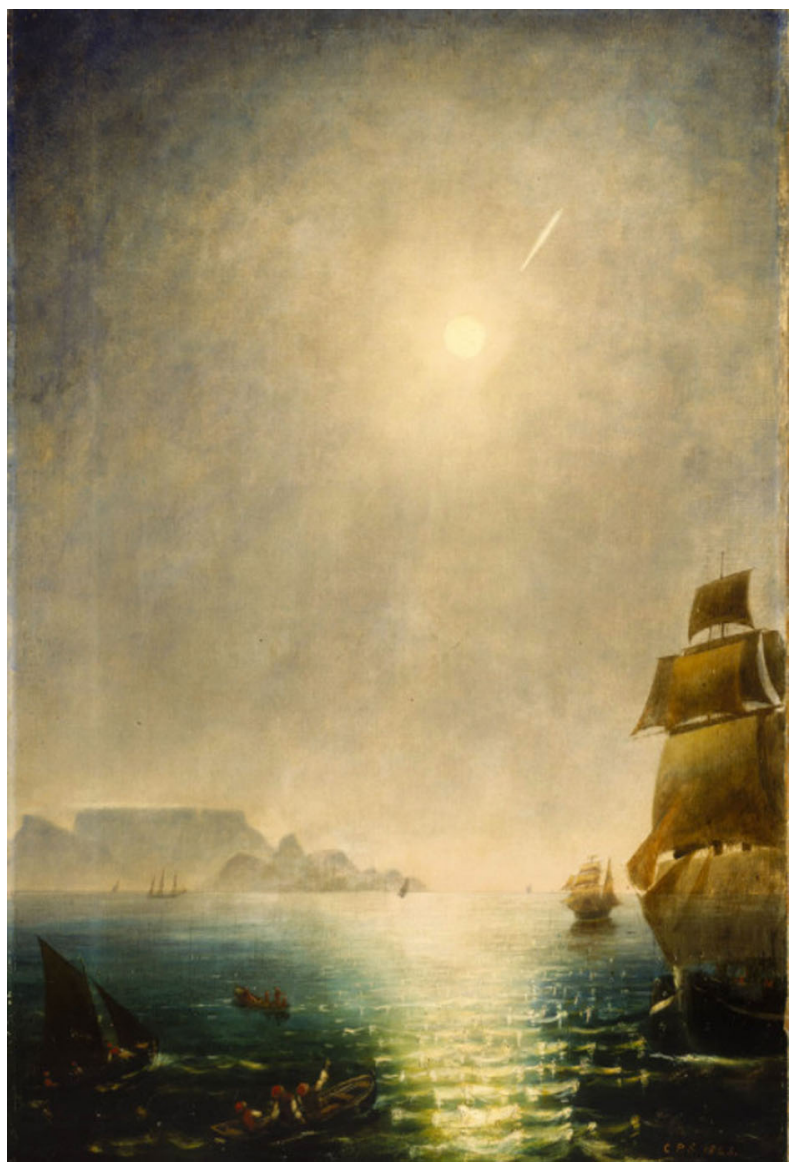

2016) are the result of repeated fragmentations of parent objects originating from some undetermined progenitor. Thus, sungrazers only represent a very small portion by mass of the total comet population. Moving farther from the Sun, we encounter smaller populations of sunskirting comets, again the result of fragmentation of unknown parent bodies, and in many cases ambiguous in nature in terms of classically asteroidal versus cometary origin. Sunskirting comets experience less extreme environments than their sungrazing counterparts, and depending on their sizes and physical properties, usually have a higher likelihood of surviving their perihelion passages. However, their proximity to the Sun places them in an environment in which chemical and physical processes occur that are quite far removed from those taking place at comets typically observed from Earth. Finally, we use the term nearSun comets to encompass all such objects that pass inside Mercury's orbit (see Sect. 1.3). While far fewer in terms of detected population, the objects that venture closer to the Sun than Mercury but not in the sunskirting distance range tend to be larger and better observed than sungrazing and sunskirting comets, and somewhat easier to observe from terrestrial observatories than comets that are only bright when extremely close to the Sun. The outermost population of near-Sun comets are still subject to highly elevated solar radiation, which drives chemical and physical processes at and near the nucleus not seen at $\sim 1 \mathrm{AU}$, and their solar wind interactions are usually easily observable. 


\subsection{The Goals of This Review}

The scientific value of near-Sun comet observations and their interpretation is enormous for the understanding of our solar system's makeup and origins. In this work, the authors attempt to provide a comprehensive summary of the current state of understanding of these bodies.

A fundamental question that should be addressed is whether all sungrazers are cometary nuclei, or parts thereof, or that some are asteroidal in nature. However, we don't have precise definitions of comets and asteroids. In fact, several small bodies in the Solar System that reside farther from the Sun share both classifications (e.g., 2060 Chiron = 95P/Chiron, 7968 Elst-Pizarro $=133 \mathrm{P} /$ Elst-Pizarro). Different bodies will be classed as comets or asteroids depending on whether the distinction is made on observational, dynamical, or compositional grounds. This is a question of semantics and we don't attempt to distinguish between the two in this paper. From an observational point of view comets are generally thought of as displaying some sort of activity, such as dust or preferably gas comae. On this basis, all sungrazers can be rightly called comets since the temperature regime they enter is sufficiently extreme to sublime refractory materials. A small, modestly bright object observed very close to the Sun in coronagraph images therefore belongs in the same group as spectacular comets that display fully developed comae and tails (see Table 2).

\subsection{Definitions}

\subsubsection{Near-Sun Comets and Sunskirters}

We use the term near-Sun comets to encompass all comets with a perihelion distance less than the perihelion of Mercury's orbit, which is $0.307 \mathrm{AU}\left(66.1\right.$ solar radii, $\left.\mathrm{R}_{\odot}\right)$. Moving inwards from this, sunskirting comets or sunskirters are terms that have been used by, for example, Sekanina and Chodas (2005), and Lamy et al. (2013), to classify comets that pass close to the Sun, but are not true sungrazers. We propose that the outer limit of this subgroup of near-Sun comets is defined by objects with a perihelion within half the perihelion of Mercury's orbit, i.e. $33.1 \mathrm{R}_{\odot}$, or $\sim 0.153 \mathrm{AU}$. This limit is coincidentally only slightly larger than the plane-of-sky field of view of the Solar and Heliospheric Observatory $(\mathrm{SOHO})$ Large Angle Spectrometric Coronagraph (LASCO) C3 instrument $\left(\sim 30 \mathrm{R}_{\odot}\right)$ (Brueckner et al. 1995). Virtually all recent sunskirting comets have been observed in SOHO-LASCO's fields-of-view. Using the above definitions, as Comet 2P/Encke has a perihelion at $0.336 \mathrm{AU}\left(72.2 \mathrm{R}_{\odot}\right)$, it falls outside the definition of a near-Sun comet, whereas Comet 96P/Machholz 1, with a perihelion distance of $0.124 \mathrm{AU}\left(26.7 \mathrm{R}_{\odot}\right)$, is therefore a near-Sun comet, and also a sunskirter. The regions encompassing the different proposed comet categories are presented in Fig. 2.

\subsubsection{Sungrazers}

Despite being a frequently-used term, no generally agreed upon definition of sungrazing comets, or sungrazers, exists. We propose that the term is defined based on the fluid Roche limit of the Sun (the point at which solar tidal forces exceed the comet's own gravity), which defines a heliocentric distance within which tides begin to disrupt the comet nucleus. This is defined by

$$
d \approx 2.44 \mathrm{R}_{\odot} \sqrt[3]{\frac{\rho_{\odot}}{\rho_{\text {comet }}}}
$$


Table 2 Notable comets relevant to this work, sorted by perihelion distance, $q$. Note the multiple occurrence of non-unique names such as "Great Southern Comet"

\begin{tabular}{|c|c|c|}
\hline Comet & $q(\mathrm{AU})$ & Notes \\
\hline \multicolumn{3}{|l|}{ Sundiver } \\
\hline C/2007 M5 SOHO & 0.0011 & Short orbital arc but $q<1 \mathrm{R}_{\odot}$ highly likely \\
\hline \multicolumn{3}{|l|}{ Sungrazers } \\
\hline C/1979 Q1 SOLWIND & 0.0048 & First Kreutz discovered from space \\
\hline C/1887 B1 (Great Southern Comet) & 0.0048 & Kreutz naked eye \\
\hline C/1963 R1 Pereyra & 0.0051 & Kreutz naked eye \\
\hline C/1880 C1 (Great Southern Comet) & 0.0055 & Kreutz naked eye \\
\hline C/1843 D1 & 0.0055 & Kreutz naked eye \\
\hline \multicolumn{3}{|l|}{ Great March Comet } \\
\hline C/2011 W3 Lovejoy & 0.0055 & Kreutz naked eye \\
\hline C/2011 N3 SOHO & 0.0060 & First Kreutz seen in EUV \\
\hline C/1680 V1 (Great Comet) & 0.0062 & Non-Kreutz, naked-eye \\
\hline C/2012 E2 SWAN & 0.0073 & Only Kreutz detected by $\mathrm{SOHO}$-SWAN \\
\hline C/1945 X1 du Toit & 0.0075 & Kreutz naked eye \\
\hline C/1882 R1 (Great September Comet) & 0.0077 & $\begin{array}{l}\text { Kreutz naked eye comet; first spectrum, Fe } \\
\text { and Ni lines seen }\end{array}$ \\
\hline C/1965 S1 Ikeya-Seki & 0.0078 & Kreutz, naked eye; many metallic lines \\
\hline C/1970 K1 & 0.0081 & Kreutz naked eye \\
\hline \multicolumn{3}{|l|}{ White-Ortiz-Bolelli } \\
\hline C/2012 S1 ISON & 0.0124 & Dynamically new (non-Kreutz) \\
\hline \multicolumn{3}{|l|}{ Sunskirters } \\
\hline C/1865 B1 (Great Southern Comet) & 0.0258 & \\
\hline C/1826 U1 Pons & 0.0269 & \\
\hline C/1962 C2 Seki-Lines & 0.0314 & \\
\hline Meyer group & $\sim 0.037$ & Typical orbit; observed by $\mathrm{SOHO}$ only \\
\hline C/1961 O1 Wilson-Hubbard & 0.0402 & \\
\hline Kracht group & $\sim 0.048$ & Typical orbit; SOHO/STEREO only \\
\hline Marsden group & $\sim 0.050$ & Typical orbit; SOHO/STEREO only \\
\hline 322P/SOHO 1 & 0.05338 & Periodic, $\mathrm{P}=3.99 \mathrm{yr}$ \\
\hline C/2002 V1 NEAT & 0.099 & Large, bright, and striated dust tail. \\
\hline 96P/Machholz 1 & 0.124 & $\begin{array}{l}\text { Periodic, } \mathrm{P}=5.28 \mathrm{yr} \text {; dynamically linked } \\
\text { to Kracht and Marsden groups }\end{array}$ \\
\hline C/1910 A1 (Great January Comet) & 0.129 & \\
\hline \multicolumn{3}{|l|}{ Other Near-Sun Comets } \\
\hline C/1973 E1 Kohoutek & 0.142 & Dynamically new \\
\hline C/2004 F4 Bradfield & 0.168 & \\
\hline C/2006 P1 McNaught & 0.171 & $\begin{array}{l}\text { Dynamically new. Extremely large, bright, } \\
\text { \& striated dust tail. Tail directly sampled } \\
\text { by Ulysses }\end{array}$ \\
\hline C/1927 X1 Skjellerup-Maristany & 0.176 & \\
\hline C/2002 X5 Kudo-Fujikawa & 0.190 & \\
\hline
\end{tabular}


Table 2 (Continued)

\begin{tabular}{lll}
\hline Comet & $q(\mathrm{AU})$ & Notes \\
\hline C/1975 V1 (West) & 0.197 & $\begin{array}{l}\text { Extremely large, bright, \& striated dust tail. } \\
\text { Observed by Skylab astronauts. Disrupted. } \\
\text { Tail directly sampled downstream by } \\
\text { Ulysses. X-ray emission discovered. }\end{array}$ \\
C/1996 B2 Hyakutake & 0.230 & $\begin{array}{l}\text { Dynamically new. Extremely large, bright, } \\
\text { \& highly structured dust tail with striae. }\end{array}$ \\
C/2011 L4 Pan-STARRS & 0.302 & \\
Select Other Comets & & Periodic, P = 3.30 yr. \\
2P/Encke & 0.336 & \\
1P/Halley & 0.586 & \\
67P/Churyumov-Gerasimenko & 1.242 & \\
\hline
\end{tabular}

where $d$ is the Roche limit in units of $\mathrm{R}_{\odot}, \rho_{\odot}$ is the mean density of the Sun, $1409 \mathrm{~kg} \mathrm{~m}^{-3}$, and $\rho_{\text {comet }}$ is the bulk density of the comet nucleus.

For an estimated comet nucleus density of $500 \mathrm{~kg} \mathrm{~m}^{-3}$, consistent with ground-based observation limits of $<600 \mathrm{~kg} \mathrm{~m}^{-3}$ (Weissman et al. 2004; Snodgrass et al. 2006) and Rosetta measurements (Sierks et al. 2015; Jorda et al. 2016; Pätzold et al. 2016) the Roche limit is $3.45 \mathrm{R}_{\odot}$ from the Sun's centre $\left(2.40 \times 10^{6} \mathrm{~km}\right.$; $\left.0.016 \mathrm{AU}\right)$. This is $2.45 \mathrm{R}_{\odot}$ from the solar photosphere; unless otherwise stated, all heliocentric distances referred to in this work are measured from the centre of the Sun, rather than altitudes above the solar photosphere.

This form of the solar Roche limit gives the distance at which a small strengthless (or fluid), synchronously-rotating body in a circular orbit will be pulled apart by solar tidal forces. Although the Roche limit definition above is not a function of time or the rate of change of solar distance, it will be a weak function of orbital eccentricity since the time spent per orbit in the strongest tidal field can vary. The spin rate and spin orientation of the body can also influence the tidal effects. See Knight and Walsh (2013) and references therein for a more detailed discussion of these effects. As a general definition, we use a perihelion distance inside or outside this heliocentric distance to differentiate between sungrazers and sunskirters.

Other possible definitions for the defining boundary of sungrazers were considered, such as the maximum heliocentric distance at which silicates sublimate, which is dependent on the silicate under consideration and modelling parameters. Marsden (2005) used a similar argument to suggest an outer perihelion limit of 10-12 $\mathrm{R}_{\odot}$; 0.0465-0.0558 AU. Also considered for the cutoff was where comets enter various regions of the solar atmosphere (e.g., photosphere, chromosphere, corona); or comets with perihelia inside the point at which their orbital speed exceeds the local solar wind speed. Given variations and uncertainties in wind speeds, this latter definition would be somewhat imprecise, but for information, mean wind models indicate that this limit is at $\sim 5 \mathrm{R}_{\odot}(0.0232 \mathrm{AU})$ for comets following parabolic trajectories (see Fig. 3).

\subsubsection{Sundivers}

Finally, we consider comets whose perihelia are $<1 \mathrm{R}_{\odot}(0.00465 \mathrm{AU})$ and would therefore enter the photosphere if they survive for long enough. We propose the term sundiver for these bodies (the terms sun-plunger and sun-impactor have been used by Brown et al. 2011, 2014). 


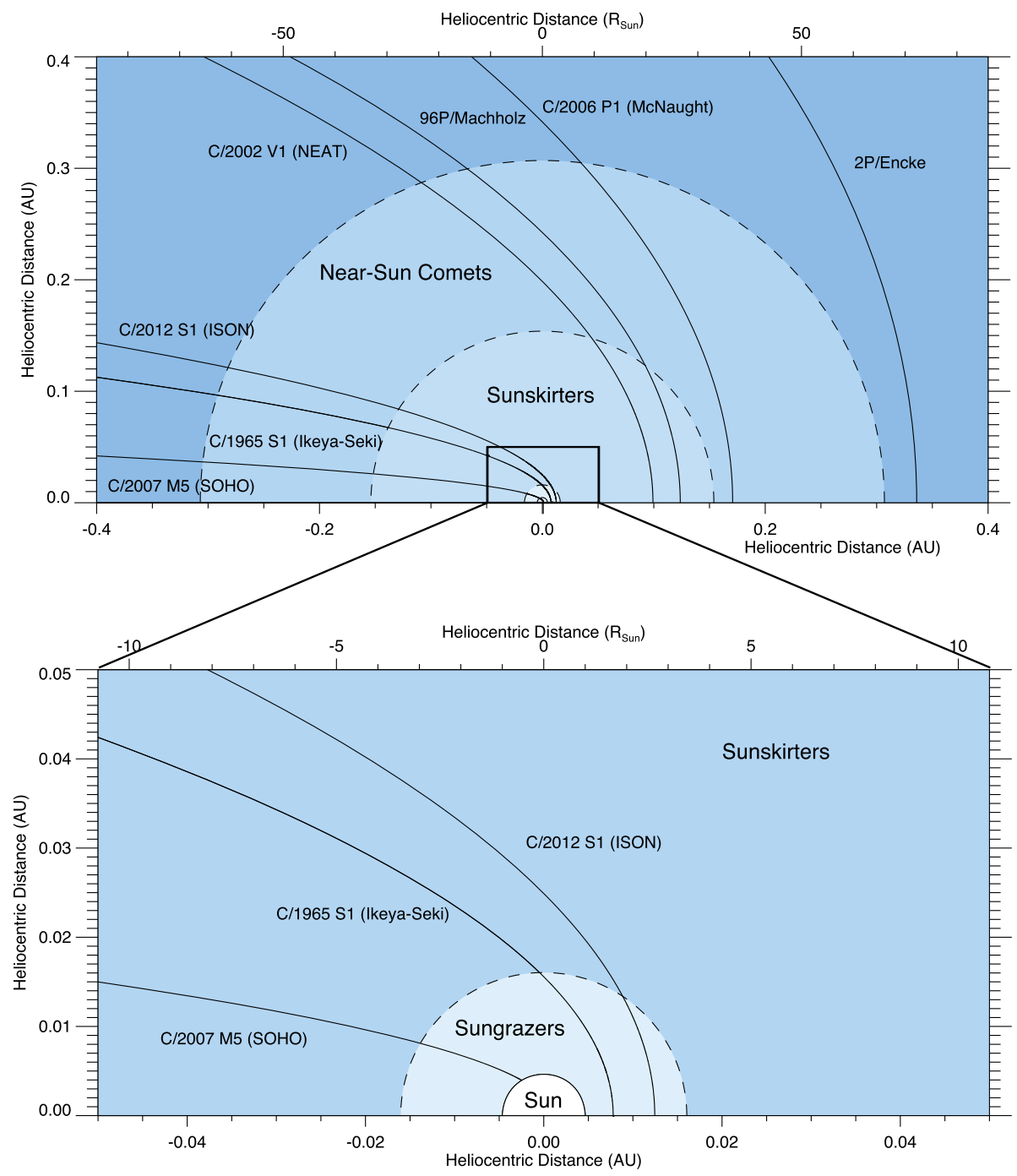

Fig. 2 Relative scales of comets' orbits in different proposed categories of near-Sun comets. The rectangle surrounding the Sun in the upper panel is shown in the lower panel. The innermost circle represents the Sun's photosphere. The orbit of C/2007 M5 (Hoffman et al. 2007) is much less well-determined than the other comets shown here, and it may or may not have had a perihelion $<1 \mathrm{R}_{\odot}(0.00465 \mathrm{AU})$, but is included as an example of a possible sundiver

Any comet with $q<\mathrm{R}_{\odot}$ will be on a sundiving orbit. We define a sundiver as one which follows such a path but also penetrates deeply enough into the low dense solar atmosphere for its mass loss and behaviour to become dominated by fluid interaction with the atmosphere rather than by insolative sublimation.

Sundivers are both observationally and theoretically rare, though they would have been very common in the early solar system. They are of great interest to both cometary and solar physics as discussed by Brown et al. (2011, 2015). For example, the spectrum of their abrupt total explosive destruction can shed light on their interior composition, while their 
Fig. 3 A comparison of heliocentric velocities of the solar wind and parabolic comets. The heliocentric speed of a parabolic comet, assuming negligible non-gravitational acceleration, is shown as a function of heliocentric distance. Also plotted is a model of solar wind speed from Lamy et al. (2003a) for a solar exobase temperature of $10^{6} \mathrm{~K}$. Inwards of $\sim 4.4 \mathrm{R}_{\odot}$ $(\sim 0.0205 \mathrm{AU})$, the heliocentric speed of a parabolic comet nucleus exceeds the expected local speed of the solar wind

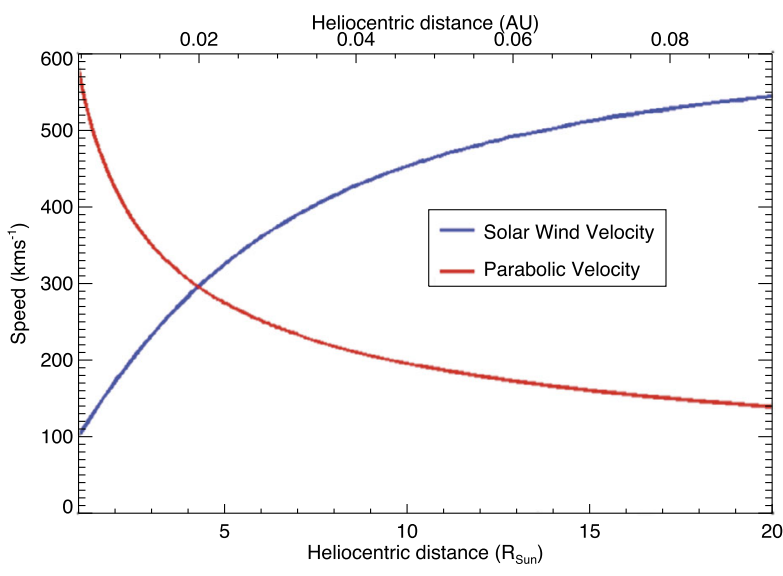

explosion deeper in the atmosphere than magnetic flares can readily generate helioseismic ripples ("Sun-quakes"), shedding light on that puzzling phenomenon (Lindsey and Donea 2008). Brown et al. $(2011,2015)$ have emphasized that the kinetic energy of very large sundivers far exceed that of the largest magnetic flares and coronal mass ejecta, CMEs, so could produce major terrestrial effects. Eichler and Mordecai (2012) suggest such an impact as the explanation of the major 7th Century isotopic abundance anomaly.

To be a sundiver, an object has to satisfy three conditions:

- $q$ must be small enough to reach the dense atmospheric regime where fluid effects (bowshock and ram pressure driven ablation and deceleration) dominate over radiative sublimation and rapidly destroy the nucleus (Weissman 1983; Brown et al. 2011, 2015). This regime's outer boundary depends on various uncertain parameters discussed in Brown et al. (2015) but is at a density $n \sim 10^{14} \mathrm{~cm}^{-3}$. This is $\sim 10^{-3}$ of photospheric values so $\sim 7$ density scale heights above the photosphere, i.e., $\sim 1000 \mathrm{~km}$, or a heliocentric distance of $\sim 1.0015 \mathrm{R}_{\odot}(0.004657 \mathrm{AU})$.

- The incident nucleus mass must be high enough, $>\sim 10^{12} \mathrm{~g}$, the exact value depending on nucleus density, latent heat, etc. (Brown et al. 2011, 2015), to avoid total sublimation when inbound.

- The overall nucleus strength must be sufficient to resist fragmentation by tidal gradient or by ablation flow pressure. Except for objects $>\sim 1 \mathrm{~km}$, quite low tensile strengths, typically on the order of $1 \mathrm{~Pa}$ (e.g. Sekanina and Yeomans 1985) far exceed self-gravity and can defeat the tidal gradient at the solar surface unless the body is a heavily cracked or a loose "rubble-pile" aggregate.

It is unclear how common sundivers are expected to be. As far as primordial comet perihelia, $q$, are concerned, Hughes (2001) reported that the observed frequency distribution $N(q)$ differential in $q$ is $q$ independent. Thus, the likelihood in the range $0<q<\mathrm{R}_{\odot}$ is the same as in $\mathrm{R}_{\odot}<q<2 \mathrm{R}_{\odot}$ etc. This would imply that the chances of a primordial comet becoming a sundiver would be $1 / 200$ of the chances of having $q$ inside 1 AU. This seems surprisingly high though if only a small fraction — say even 0.1 - of such comets were sufficiently massive and strong to survive sublimation and fragmentation, and to reach the photosphere, only 1 in 2000 of them would be sundivers.

A sundiver must either be on its first approach to the Sun or have had its $q$ value reduced by orbital perturbation, otherwise it would have impacted during the preceding perihelion 
passage. Marsden $(1967,2005)$ stated, when referring to Kreutz group comets (Sect. 3.1.1) "... it is certainly possible —indeed probable — that some of them [the Kreutz Group] hit the Sun". Solar impactors should exist and should be observable with modern instrumentation, but they are rare.

We note that $\mathrm{C} / 2007 \mathrm{M} 5 \mathrm{SOHO}$, had a derived orbit where $q=0.0011 \mathrm{AU}$, or $0.24 \mathrm{R}_{\odot}$ (Hoffman et al. 2007). The comet was only observed during 14 observations over a 4.5-hour period in $\mathrm{SOHO}$-LASCO C2. It was extremely small and stellar in appearance, and faded below the detection threshold more than four hours before its predicted impact with the Sun. Despite observational uncertainties inherent to SOHO-LASCO data reductions, there was an unusually high confidence in the trajectory of the comet (Marsden, personal comm.). However, there is no evidence that this object actually reached the solar photosphere; it most likely was destroyed shortly after fading from view.

Five SOHO-observed Kreutz comets published prior to 2011 have $q<0.004 \mathrm{AU}$ (0.85 R $\odot$ ); viz. C/2007 C3, C/2007 C13, C/2009 D5, C/2009 E2, and C/2009 U9. The first four were all observed by both $S O H O$ and STEREO so have orbits much better constrained than typical $\mathrm{SOHO}$-only Kreutz members. These objects all belong to an period when Marsden (private comm.) strongly preferred to force orbits to have $q \geq \mathrm{R}_{\odot}$, so it is likely that other comets had orbits with $q<\mathrm{R}_{\odot}$ but were rejected at the time. Since Marsden's death in 2010 , there have been more orbits with $q<\mathrm{R}_{\odot}$ published by others. This represents a much greater fraction of the published Kreutz orbits than during the Marsden era, but these orbits also demonstrate considerably more scatter in all elements than those published by Marsden. Thus it is difficult to draw firm conclusions about the true frequency of sundivers due to the apparent human bias in selecting the "best" orbit for very sparsely observed objects. One additional potential sundiver was 1979 XI = C/1979 Q1 SOLWIND (Fig. 4, cf. Sekanina 1982b; Weissman 1983), but the orbital uncertainties resulting from limited and low-resolution astrometry preclude its definitive assignment to this class.

\subsection{The Near-Sun Environment}

We address in detail the conditions experienced by comets that venture close to the Sun later in this work. When considering a comet in this environment compared to $\sim 1 \mathrm{AU}$, it should be borne in mind that comet nuclei are still $10^{17}$ times denser than even the inner solar corona. However, sungrazers' tenuous tails can interact strongly with the solar corona. That interaction can provide diagnostics for the comet, such as its size and composition, and for the corona itself, such as local magnetic field strength and orientation, density, temperature and outflow speed.

The corona can be roughly divided into three domains: closed magnetic field regions, open magnetic field regions containing the beginnings of the slow solar wind, and open magnetic field regions with fast solar wind. The temperatures are estimated to be around $(1-2) \times 10^{6} \mathrm{~K}$ in these structures, and magnetic fields of $\sim 1 \mathrm{G}(100 \mu \mathrm{T})$ at $1.3 \mathrm{R}_{\odot}$ (0.00605 AU), decreasing to $\sim 0.01 \mathrm{G}$ at $10 \mathrm{R}_{\odot}(0.0465 \mathrm{AU})$. Densities drop quickly from $10^{7} \mathrm{~cm}^{-3}$ at $1.5-2 \mathrm{R}_{\odot}(0.0070-0.0093 \mathrm{AU})$ to $10^{3}-10^{4} \mathrm{~cm}^{-3}$ at $10 \mathrm{R}_{\odot}(0.0465 \mathrm{AU})$, while the outflow speed in the slow wind increases from perhaps 20 to $200 \mathrm{~km} \mathrm{~s}^{-1}$ and that in the fast wind from 100 to $600 \mathrm{~km} \mathrm{~s}^{-1}$. The closest in situ sampling of the solar wind to date was carried out by the West German/NASA Helios probes, which reached 0.29 AU from the Sun. The Solar Orbiter and Parker Solar Probe missions will explore this domain, as described in Sect. 13.3.1. The question of the dominant physical processes and regimes of mass, momentum, and energy loss for near-Sun comets is addressed in Sect. 6. 


\subsection{Comet Groups}

Most sungrazers and sunskirters belong to one of a few distinct groups of comets sharing similar orbital elements ("families" is also commonly used, but for consistency we use "groups" throughout this review). The overwhelming majority have been discovered and observed only by space-based solar observatories. We introduce them briefly here and discuss them in more detail in Sect. 3.1.

By far the most common and best-studied association is the Kreutz group (Kreutz 1888, 1891 ), which accounts for $\sim 85 \%$ of $\mathrm{SOHO}$-discovered comets, and is the only known group of sungrazers. Its members include some of the most spectacular comets in recorded history, including C/1882 R1 (The Great Comet of 1882), C/1965 S1 Ikeya-Seki, and recently, C/2011 W3 Lovejoy. As of 2017 October, it also includes $>2,900$ small ( $\lesssim 100 \mathrm{~m}$ ) comets discovered by space-based coronagraphs. Kreutz sungrazers are thought to have fragmented from a single parent body as indicated by their very similar orbital elements.

Other less populated groups, all sunskirters, are the Meyer, Marsden and Kracht groups; the latter two are seemingly of common origin, cf. Ohtsuka et al. (2003), Sekanina and Chodas (2005). Most sunskirting comets appear to survive, though there is some ambiguity due to a short observing arc that does not always encompass their perihelion. The orbital element groupings of these objects strongly suggest a limited number of progenitor objects.

Table 2 lists a number of comets in each of the classifications just discussed. It is not intended to be all-inclusive, but does include all comets discussed in detail in this paper. We include brief notes for some comets in the table, and discuss some in more detail as appropriate elsewhere in the text. For specific details about particular comets observed prior to 1982 and listed below, the reader is directed to Kronk (1999, 2003, 2007), Kronk and Meyer (2010). The table is ordered by increasing perihelion distance, $q$. All comets with original reciprocal semi-major axis, $1 / a_{0}$, consistent with being dynamically new, are noted (e.g., $1 / a_{0}<0.0001 \mathrm{AU}^{-1}$; Levison 1996). All values of $q$ and $1 / a_{0}$ are from Marsden and Williams (2008) when possible; newer objects are from JPL Horizons and the Minor Planet Center.

\subsection{Case Studies Presented in this Paper}

In recent years, a significant increase in information on sungrazers has resulted from the apparitions of comets C/2011 N3 SOHO, C/2011 W3 Lovejoy, and C/2012 S1 ISON; with the latter in particular being one of the most broadly studied comets in history. We provide here a brief overview of each of these objects, but defer detailed descriptions of their science results to relevant subsections of this paper. All other comets referenced in this document will be introduced in relevant sub-sections.

\subsubsection{C/2011 N3 SOHO}

In July 2011, a Kreutz-group sungrazing Comet C/2011 N3 SOHO (Uchina et al. 2011) became the first comet directly witnessed to undergo destruction in the low solar corona. The comet was discovered in coronagraph images recorded by the SOHO-LASCO C3 instrument on 2011 July 4, and quickly brightened to an estimated magnitude of $\sim 1$ by 2011 July 5.8 (Battams and Williams 2011). The comet became visible in Extreme Ultraviolet (EUV) images of the Sun recorded by the Solar Dynamics Observatory (SDO)-Atmospheric Imaging Assembly (AIA) instrument on 2011 July 6, where its fragmentation and destruction were recorded as it approached to a heliocentric distance of $1.146 \mathrm{R}_{\odot}, 0.00533 \mathrm{AU}$, or just $\sim 10^{5} \mathrm{~km}$ from the solar photosphere (Schrijver et al. 2012). 


\subsubsection{C/2011 W3 Lovejoy}

Kreutz-group sungrazer C/2011 W3 Lovejoy (Lovejoy and Williams 2011) was first identified in ground-based images by amateur astronomer T. Lovejoy on 2011 November 27. This was the first ground-based discovery of a sungrazing comet since C/1970 K1 (White-OrtizBolelli) in 1970. With approximately 15 days of warning, it was possible to coordinate a number of space-based observatories to alter their observing plans and adapt their observing capabilities to accommodate observations of the comet. Consequently, observations were recorded by numerous instruments aboard the SOHO, Proba-2, STEREO, SDO, and Hinode spacecraft (Sect. 2). After perihelion, the comet was only observed as a headless tail that slowly dissipated during the following weeks (Knight et al. 2012), and is presumed to have been destroyed. The comet may have disintegrated near its closest point to the Sun, but Sekanina and Chodas (2012) and Gundlach et al. (2012) suggest that the nucleus may have survived post-perihelion for hours to a few days before disrupting.

\subsubsection{C/2012 S1 ISON}

Comet C/2012 S1 ISON was discovered on 2012 September 21, in images recorded by the International Scientific and Optical Network (ISON) telescopes. It was soon determined to be a sungrazing comet with a perihelion distance of $0.01244 \mathrm{AU}\left(2.7 \mathrm{R}_{\odot}\right)$ to be reached on 2013 November 28. Due to an unprecedented lead time before perihelion, ISON became the subject of a broad and global observing campaign (http://www.isoncampaign.org/) with numerous ground- and space-based observatories making observations (e.g., Li et al. 2013; Meech et al. 2013; O'Rourke et al. 2013; Bonev et al. 2014; Combi et al. 2014; Cordiner et al. 2014; Hines et al. 2014; Shinnaka et al. 2014; Knight and Schleicher 2015; Schmidt et al. 2015). A fortuitous route through the inner solar system took the comet relatively close to Mars and Mercury, enabling observations from planetary spacecraft operating at those planets, before passing through the fields of view of the STEREO and SOHO solar observatories. As the comet approached the Sun in November 2013, it was observed to brighten extremely rapidly and then began to fade in the hours preceding perihelion (Knight and Battams 2014). An apparently cometary object was seen to emerge from perihelion in coronagraph observations, but with an increasingly diffuse nature. Numerous investigations have concluded that the comet disrupted before perihelion (e.g., Combi et al. 2014; Knight and Battams 2014; Sekanina and Kracht 2014; Steckloff et al. 2015b). There were no definite post-perihelion observations of Comet C/2012 S1 ISON beyond the STEREO-A-SECCHI HI1 field of view, when a surviving nucleus would have been at $\sim 0.18 \mathrm{AU}\left(\sim 39 \mathrm{R}_{\odot}\right)$.

\section{Instruments and Facilities Used}

\subsection{Introduction}

The first telescopically discovered sungrazer was C/1680 V1, the motion of which Newton used to verify Kepler's Laws and Newtonian gravity in his PhilosophiceNaturalis Principia Mathematica and was the motivation for deriving an inverse-square law of gravity (e.g. Heidarzadeh 2008). Telescopic observations primarily employed optical imaging, but optical spectra were acquired of Kreutz Comets C/1882 R1 (The Great September Comet of 1882) and C/1965 S1 Ikeya-Seki (see review by Marsden 1967; also Kronk 1999, 2003, 2007; 


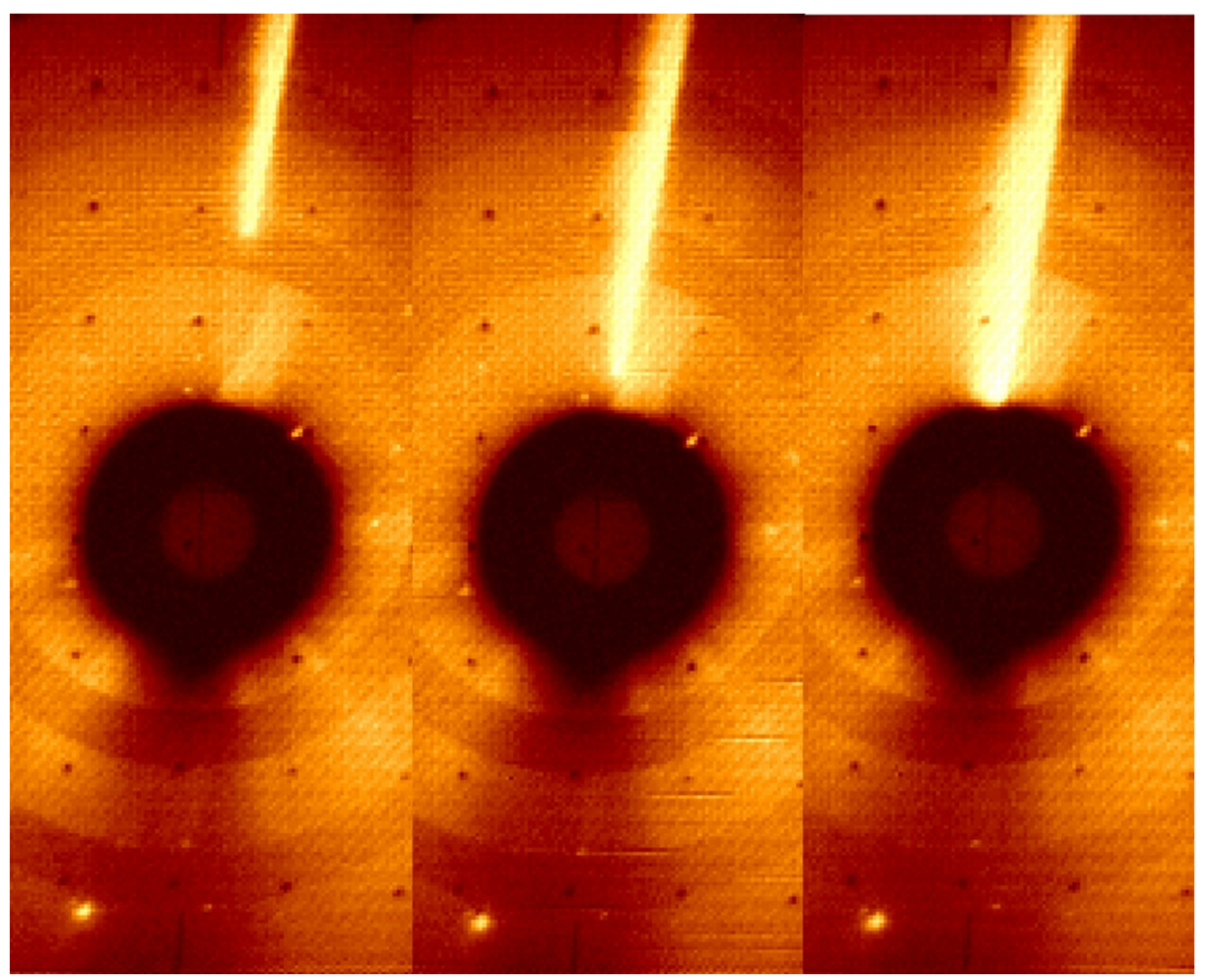

Fig. 4 A series of three images of Kreutz-group Comet C/1979 Q1 SOLWIND, observed by the SOLWIND white light coronagraph aboard the USAF P78-1 satellite on August 30, 1979. This was the first of ten comets discovered by the SOLWIND instrument

Kronk and Meyer 2010). The first two infrared (IR) observations of comets were of nearSun Comets C/1927 X1 Skjellerup-Maristany (see Marcus 2013a and references therein) and C/1965 S1 Ikeya-Seki (Becklin and Westphal 1966).

Studies of near-Sun comets are now primarily based on data from space- and groundbased instruments designed for the study of the Sun, its corona, and the inner heliosphere. The rate of discovery of near-Sun comets and the manner in which they are studied has changed tremendously in the past few decades: the advent of space-based solar observatories revolutionized our knowledge of the populations of small comets near the Sun. A total of 20 near-Sun comets were discovered in US Air Force P78-1/SOLWIND (Fig. 4) and NASA Solar Maximum Mission (SMM) coronagraphic images (Fig. 5) during 1979-1989 (reviewed by Marsden 1989; MacQueen and St. Cyr 1991, plus later archival discoveries by R. Kracht, e.g., Kracht and Marsden 2005a). Much more sensitive coronagraphs and heliospheric imagers onboard the joint ESA/NASA SOHO (LASCO, since 1996) and the twin NASA Solar Terrestrial Relations Observatory, STEREO (Solar Earth Connection Coronal and Heliospheric Investigation, SECCHI, since 2006), have imaged more than 3,200 near-Sun comets as of 2017 October (see comprehensive papers by Biesecker et al. 2002; Marsden 2005; Knight et al. 2010; Lamy et al. 2013; Battams and Knight 2016).

Novel technologies launched on $\mathrm{SOHO}$ and later missions have expanded investigations into the UV and EUV regions. Regular spectroscopic imaging of bright Kreutz comets was carried out by SOHO-Ultraviolet Coronagraph Spectrometer (UVCS) during 1996-2012 
Fig. 5 Kreutz group Comet C/1988 Q1 SMM, lower right, in images recorded by the white-light

Coronagraph/Polarimeter aboard the Solar Maximum Mission observatory on 1988 August 21. This was the fourth of ten Kreutz comets discovered by SMM. The position of the Sun's disk is traced by the yellow points

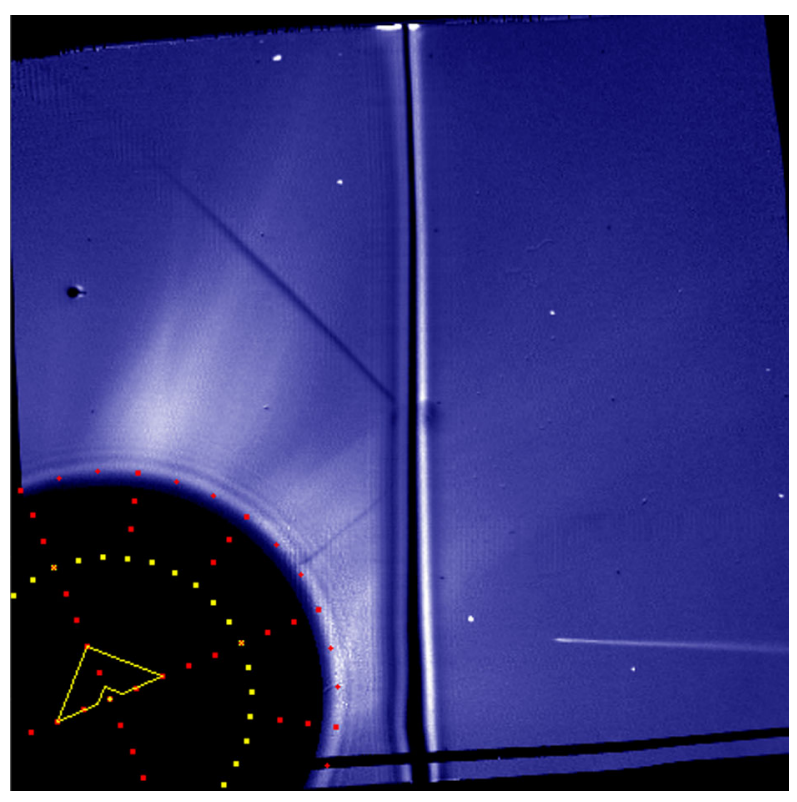

(see review by Bemporad et al. 2007). SOHO-Solar Wind ANisotropy experiment (SWAN) (Bertaux et al. 1995) data led to the discovery of sungrazer C/2012 E2 SWAN. The SDOAIA (Lemen et al. 2012), STEREO-SECCHI Extreme Ultraviolet Imager, EUVI (Wuelser et al. 2004), JAXA Hinode XRT (Golub et al. 2007), and ESA Proba-2 Sun Watcher with Active Pixels and Image Processing, SWAP (Seaton et al. 2013), all observed at least one sungrazing comet in the UV or EUV. To the authors' knowledge, the NASA Transition Region and Coronal Explorer (TRACE) mission, which had a circular field of view of width $<0.5 \mathrm{R}_{\odot}$ at the Sun $(0.0023 \mathrm{AU})$, did not observe any comets during its 12 years of operation.

Overwhelmingly, coronagraph instruments (e.g., Fig. 6) have proved the most successful at detecting sungrazing comets, with their optics designed such that direct sunlight is blocked by way of a solid occulting disk, enabling the detection of signals a factor of $10^{10}-10^{11}$ fainter than direct sunlight.

\subsection{Visible Light Telescopes}

\subsubsection{Early Space-Based Solar Observatories}

Coronagraph instruments flown on the 7th Orbiting Solar Observatory (OSO-7) mission (Koomen et al. 1970) and the Skylab mission (MacQueen et al. 1974) yielded no positive detections of sungrazing comets, though other instruments on the latter did make successful far-UV observations of near-Sun Comet C/1973 E1 (Kohoutek) (Page 1974). Knight et al. (2010) concluded that the lack of Skylab detections was not statistically unusual given its sensitivity and the rate of small comets observed by SOHO from 1996-2005.

Space-based observations of near-Sun comets began with the surprise discovery of C/1979 Q1 SOLWIND in coronagraph images recorded by the P78-1/SOLWIND satellite in August 1979 (Howard et al. 1981). Instrument scientists at the US Naval Research Laboratory determined an approximate orbital solution for the comet, recognizing it as a member 
Fig. 6 Schematic overview of SOHO-LASCO's C2 occulter. The occulting disk blocks the vast majority of sunlight, revealing much fainter features such as coronal mass ejections (pictured) or, on occasion, comets

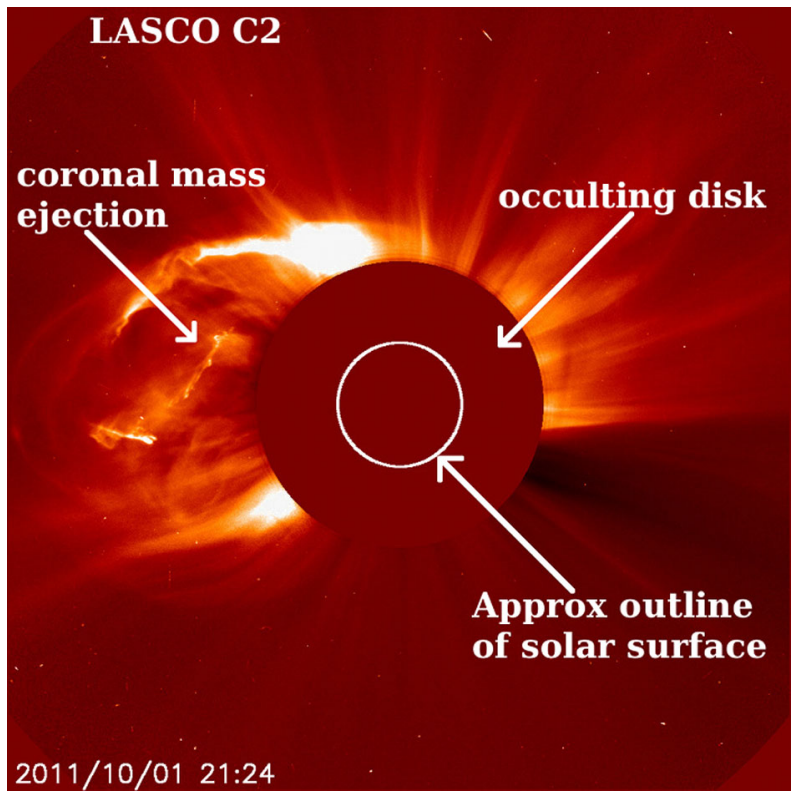

of the Kreutz group. This was the first Kreutz comet discovered since C/1970 K1 WhiteOrtiz-Bolelli and, perhaps more significantly, its finding was the first space-based discovery of a comet.

Speculation still surrounds the orbital parameters of C/1979 Q1, with the very low spatial resolution and temporal cadence of SOLWIND images leaving a range of possible solutions, some of which would results in classification as a sundiver (Sekanina 1982a; Marsden 1989). This comet was not observed to survive perihelion. Analysis of the SOLWIND data during that period does indicate a dramatic brightening of the solar corona in the hours following the comet's passage, but the data are of insufficient quality to resolve the ambiguity of whether the brightness enhancement resulted from the redistribution of material from an impacting comet, or simply a projection effect through the comet's tail.

During its fully operational period of 1979-1984, SOLWIND yielded a further nine comet discoveries (e.g., Sheeley et al. 1982), four of which were archival (Kracht and Marsden 2005a,b,c). All but one (Kracht and Marsden 2005b) of these belonged to the Kreutz group. The satellite was destroyed in 1985 as part of a planned United States Air Force exercise.

In 1980, the Solar Maximum Mission (SMM) launched, carrying another coronagraph. The satellite suffered attitude control and instrument electronics failures until its repair in orbit in 1985. It discovered ten Kreutz-group comets between 1987 and 1989 (reviewed by MacQueen and St. Cyr 1991).

The advent of space-based coronagraphs thus more than doubled the population of cataloged sungrazers during the period 1979-1989, implying that the Kreutz group was substantially more populous than the previous century of ground-based discoveries had indicated (cf. Marsden 1989). Confirmation of this came soon after the launch of SOHO in 1995. 
Table 3 SOHO-LASCO and STEREO-SECCHI white-light telescope and filter information, with typical observing parameters. Exposure times, image sizes, and frequency of images have varied throughout the lifetimes of both missions

\begin{tabular}{llcllll}
\hline Spacecraft & Telescope & $\begin{array}{l}\text { Field of } \\
\text { view }^{\mathrm{a}}\left(^{\circ}\right)\end{array}$ & $\begin{array}{l}\text { Pixel scale } \\
\left({ }^{\prime \prime} \mathrm{pixel}^{-1}\right)\end{array}$ & $\begin{array}{l}\text { Bandpass } \\
(\mathrm{nm})\end{array}$ & $\begin{array}{l}\text { Exposure time } \\
(\mathrm{sec})\end{array}$ & $\begin{array}{l}\text { Frequency } \\
\left(\mathrm{day}^{-1}\right)\end{array}$ \\
\hline SOHO & $\mathrm{C} 2$ & $0.3-1.6$ & 11.9 & $\mathrm{~B}, \mathrm{O}, \mathrm{R}^{\mathrm{b}}$ & 25 & $96-120$ \\
SOHO & $\mathrm{C} 3$ & $1.0-8.0$ & 56.0 & $\mathrm{C}, \mathrm{B}, \mathrm{O}, \mathrm{R}^{\mathrm{b}}$ & 19 & $96-120$ \\
STEREO & COR1 & $0.4-1.0$ & 3.8 & $650-660$ & $0.7-3$ & $144-864$ \\
STEREO & COR2 & $0.5-4.0$ & 14.7 & $650-750$ & $2-6$ & $96-168$ \\
STEREO & HI1 & $4.0-24.0$ & 70.0 & $630-730^{\mathrm{c}}$ & $1200^{\mathrm{d}}$ & 36 \\
STEREO & HI2 & $20.7-90.7$ & 240.0 & $400-1000$ & 4950 & 12 \\
\hline
\end{tabular}

${ }^{\mathrm{a}}$ Annular fields of view centered on the Sun except for HI1 and HI2 which are offset by $14.0^{\circ}$ and $55.7^{\circ}$, respectively, along the Earth-Sun line as viewed by the spacecraft

${ }^{\mathrm{b}} \mathrm{C}=$ Clear $(400-850 \mathrm{~nm}), \mathrm{B}=$ Blue $(420-520 \mathrm{~nm}), \mathrm{O}=$ Orange $(540-640 \mathrm{~nm}), \mathrm{R}=\operatorname{Red}(730-835 \mathrm{~nm})$. The vast majority of images are $\mathrm{C} 2$ orange or $\mathrm{C} 3$ clear

${ }^{\mathrm{c}}$ Also has significant blue transmission (Bewsher et al. 2010)

${ }^{\mathrm{d}}$ On-board combination of 40 exposures each of $30 \mathrm{sec}$

\subsubsection{Post-1995 Era}

No single telescope has made a greater impact on the study of sungrazing comets than LASCO aboard SOHO. SOHO resides in a halo orbit about the Earth-Sun L1 Lagrange point, providing an uninterrupted view of the Sun and its environment. The LASCO instrument (Brueckner et al. 1995) comprises three annularly occulted coronagraph telescopes known as C1, C2 and C3, covering increasingly wide regions of the solar corona.

C1 only operated during 1996-1998, and as it used only filters centered on forbidden emission lines that are not expected in comets, it never detected any sungrazing comets. $\mathrm{C} 2$ and $\mathrm{C} 3$ are broadband, externally occulted coronagraphs spanning the regions of 2.0-6.0 $\mathrm{R}_{\odot}(0.009-0.028 \mathrm{AU})$ and 3.7-30 $\mathrm{R}_{\odot}(0.017-0.139 \mathrm{AU})$, respectively. Each telescope is equipped with a $1024 \times 1024$ pixel CCD and a selection of filters and polarizers, summarized in Table 3. These coronagraphs have been responsible for almost all of SOHO's over 3,400 sungrazing and near-Sun comet discoveries. LASCO C2 has proven the most effective at comet detections, primarily due to its slightly higher sensitivity and smaller pixel scale compared C3, though strong seasonal variations in detection rates occur as a result of SOHO's orbit around the Sun (Knight 2008; see Sect. 6.1 of Knight et al. 2010 for additional discussion).

In October 2006, the two STEREO spacecraft were launched. These near-identical solar observatories were placed into Earth-like orbits at 1 AU, with STEREO "Ahead" (or "A") leading Earth, and STEREO "Behind" (or "B") trailing Earth. The respective velocities of the spacecraft are such that they separate from Earth at a rate of approximately $22^{\circ}$ per year (thus the separation between the two spacecraft increases at $\sim 45^{\circ}$ annually), reaching solar conjunction in early 2015 . The evolving STEREO observing geometry has enabled stereoscopic observations of sungrazing and near-Sun comets with the telescopes that comprise STEREO's SECCHI instrument suite, sometimes in combination with SOHO-LASCO, e.g. Fig. 7. STEREO-B has not carried out scientific operations since October 2014.

SECCHI comprises five telescopes (Fig. 8) that observe the solar atmosphere, the corona, and the heliosphere out to beyond Earth's orbit for the nominal mission. These are an EUV 


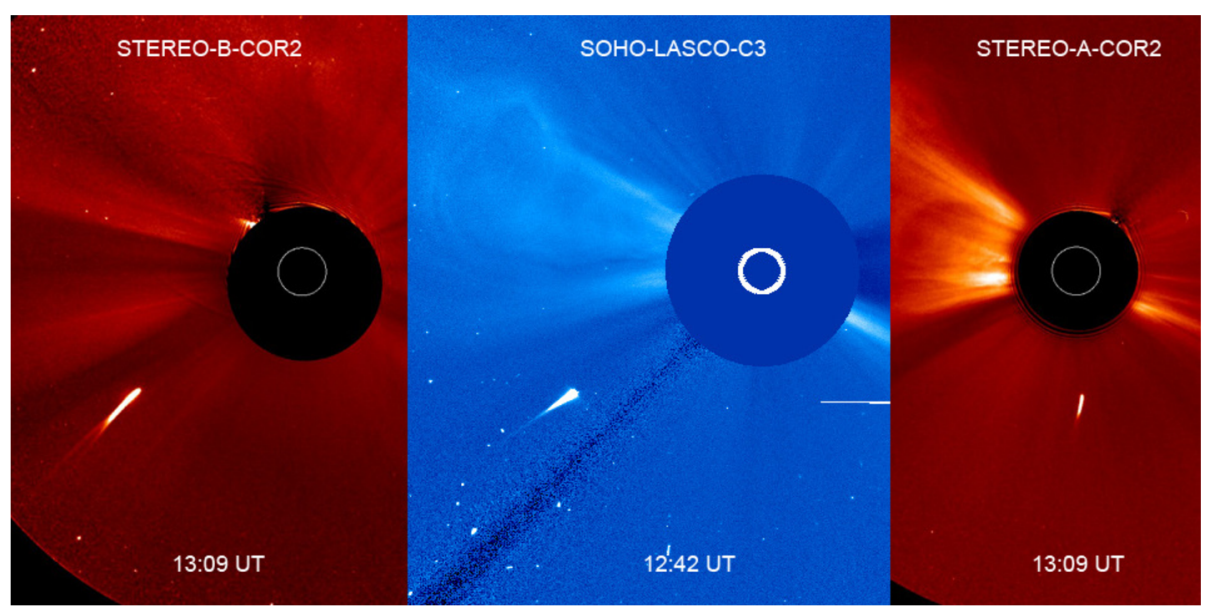

Fig. 7 Kreutz group Comet C/2010 E6 STEREO observed almost simultaneously by coronagraphs on STEREO-B, SOHO, and STEREO-A, on 2010 March 12. STEREO-B and -A were $71.5^{\circ}$ and $66.1^{\circ}$ behind and ahead of Earth in its orbit, respectively. The images have been scaled such that the Sun's disk (white circle) is the same size

Fig. 8 Fields of view of SECCHI coronagraphs and heliospheric imagers. The circular HI-2 field of view extends to the left of this diagram

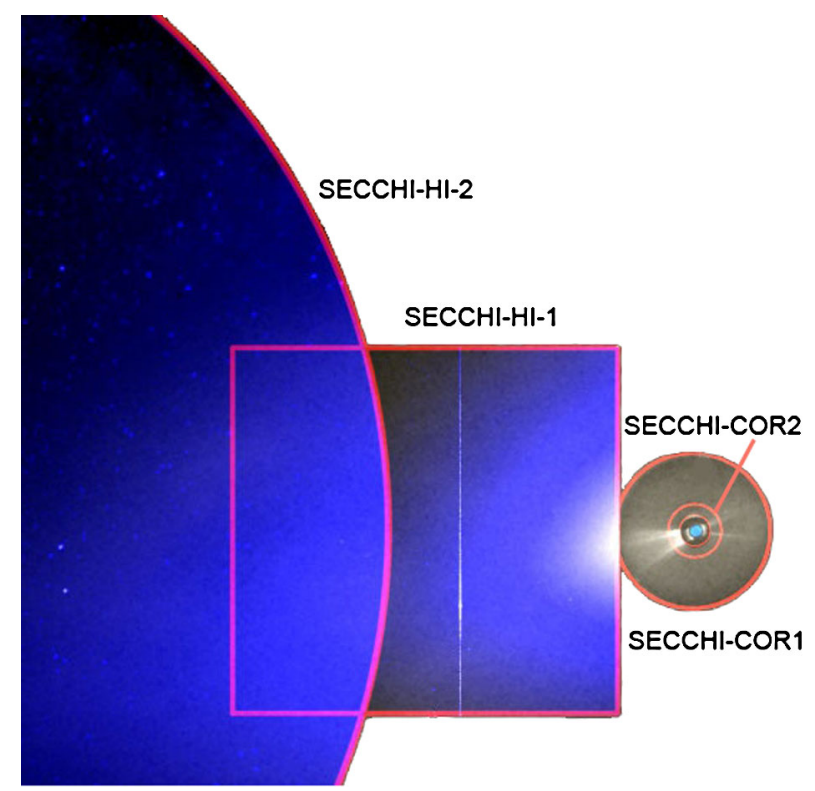

disk imager (EUVI, 1-1.7 $\left.\mathrm{R}_{\odot} ; 0.005-0.008 \mathrm{AU}\right)$, two white-light Lyot coronagraphs: COR1 (1.4-4 $\left.\mathrm{R}_{\odot} ; 0.006-0.019 \mathrm{AU}\right)$, and COR2 (2-15 $\left.\mathrm{R}_{\odot} ; 0.009-0.070 \mathrm{AU}\right)$ and two heliospheric imagers (HI1, HI2) which observe approximately square regions offset from the Sun and together cover near-ecliptic space from 12 to $318 \mathrm{R}_{\odot}(0.056-1.479 \mathrm{AU})$ (Howard et al. 2008). Angular fields of view are given in Table 3.

Additional information on both the SOHO and STEREO coronagraphs, such as bandpasses, exposure times, cadences, etc. are given in Table 3 and Fig. 9, and many fields of 


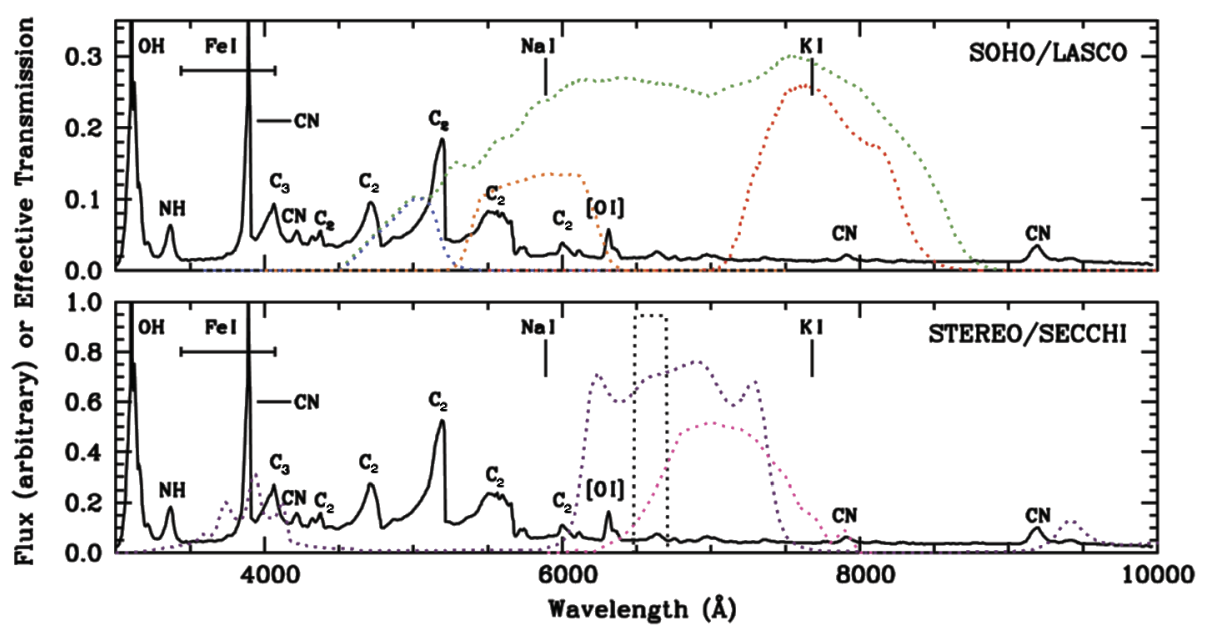

Fig. 9 SOHO-LASCO and STEREO-SECCHI bandpasses overlaid on a typical comet spectrum. The y-axis shows flux in arbitrary units (for the comet spectrum) and effective transmissions (folding in quantum efficiency and filter response) for the filters. The solid black line is the spectrum of Comet 8P/Tuttle (provided by S. Larson), and filters are shown as colored dotted lines. The SOHO-LASCO filters (Brueckner et al. 1995) are shown in the top panel. Green is the clear filter, blue is the blue filter, orange is the orange filter, and red is the "deep red" filter. The STEREO-SECCHI bandpasses (Bewsher et al. 2010; A. Vourlidas private communication, 2012; B. Thompson, private communication, 2012) are shown in the bottom panel. Black is HI1, purple is COR2, and pink is COR1. Note that the bandpasses for the same telescopes on STEREO-A and STEREO-B are effectively identical. Common comet gas emission bands and the locations of significant elemental emission lines seen in the spectrum of sungrazer C/1965 S1 Ikeya-Seki (Preston 1967; Slaughter 1969) are also labeled

view are shown in Fig. 8. While the STEREO-SECCHI telescopes are technologically superior to those on $S O H O$-LASCO, overall many fewer comets have been discovered with STEREO than with $S O H O$. This is due to three primary factors:

- The SECCHI bandpasses are generally narrower than LASCO bandpasses and they do not include the NaI doublet that makes many near-Sun comets very bright. (See Fig. 9).

- The SECCHI fields of view are sub-optimal for detecting Kreutz group members, with the Kreutz orbit only passing through the HI1 fields of view seasonally and missing HI2 altogether.

- The full resolution SECCHI data are transmitted to Earth after a delay of several days, by which point most comets have already been discovered in LASCO images.

A notable limitation of coronagraphic discovery of a comet is that first detection inherently occurs close to the Sun and typically hours or, at most days, prior to the comet's vaporization. Ground-based surveys for sungrazers prior to their appearance in $\mathrm{SOHO}$ and STEREO images have thus far been unsuccessful (see Sect. 2.5). However, several nonsungrazers and three sungrazers discovered in advance-C/2011 W3 Lovejoy, C/2012 E2 SWAN, and C/2012 S1 ISON-were discovered early enough prior to perihelion to allow dedicated observations by LASCO using its color filters.

The Solar Optical Telescope (SOT) aboard the Japanese-led Hinode mission has detected one comet, observing Comet C/2011 W3 Lovejoy as a point source and providing an accurate position just before the comet passed behind the Sun (McCauley et al. 2013). The Solar Mass Ejection Imager, SMEI, aboard the US Navy Coriolis satellite (Jackson et al. 2004), 
could also observe the comae and tails of active comets as they appeared in very wide angle maps of the sky. This included occasional observations of near-Sun comets such as C/2004 F4 Bradfield (Kuchar et al. 2008).

\subsection{Ultraviolet Telescopes}

The SWAN instrument aboard $\mathrm{SOHO}$ measures the Ly $\alpha$ brightness over most of the sky with very high sensitivity to detect backscattered photons from interstellar hydrogen atoms within the heliosphere. SWAN has observed Ly $\alpha$ from many comets, particularly near perihelion (e.g., Bertaux et al. 2014). It can also detect the shadows of large comets such as C/1995 O1 Hale-Bopp in the interplanetary Ly $\alpha$ (Lallement et al. 2002). SWAN observed C/2012 S1 ISON through its outburst late in 2013 November (Combi et al. 2014), and eight near-Sun comets have been discovered in its images (e.g., Combi et al. 2011), notably including one Kreutz sungrazer, C/2012 E2 SWAN (Bezugly et al. 2012). It has also observed numerous comets with $q=0.18-1.55$ AU (39-333 $\mathrm{R}_{\odot}$ ) (e.g., Mäkinen et al. 2000).

$\mathrm{SOHO}$ also carries UVCS, which was designed to observe the solar corona between 1.5 and $10 \mathrm{R}_{\odot}(0.007-0.046 \mathrm{AU})$ at wavelengths from 500 to $1350 \AA$. This operated from 1996 through 2013 (Kohl et al. 1995, 2006). Its 42' long slit could be placed so that a comet would cross it, which for most sungrazers required discovery in SOHO-LASCO images, computation of the orbit, and planning of the UVCS observation within half a day or less. A series of spectra would be obtained, and since the comet's speed was known, the time series could be converted into a spatial image. UVCS observed 10 Kreutz comets (Bemporad et al. 2007) along with four others near perihelion; C/1997 H2 SOHO (Mancuso 2015), 2P/Encke (Raymond et al. 2002), 96P/Machholz 1, and C/2002 X5 Kudo-Fujikawa (Povich et al. 2003). Most recently it observed C/2011 W3 Lovejoy. UVCS observed the Lyman lines along with lines of O I, C II, C III, Si III and N V, or in chemical notation, O, C ${ }^{+} \mathrm{C}^{2+}, \mathrm{Si}^{2+}$, $\mathrm{N}^{4+}$, respectively, to obtain outgassing rates and cometary abundances. It also provided a probe of the coronal density, temperature, and outflow speed at specific points along the comet trajectory, free of the line-of-sight integration that limits most remote sensing coronal observations. An example of joint comet observations by SOHO-LASCO and -UVCS is given in Fig. 10.

$S D O$ was launched in 2010, and it operates in a circular, geosynchronous orbit. Using its AIA instrument (Lemen et al. 2012), SDO is designed to image the Sun at high spatial and temporal resolution in 10 narrow bands in the EUV and UV, mostly centered on lines from highly ionised iron (Lemen et al. 2012). Its 0.6" pixels cover a field of view out to $1.3 \mathrm{R}_{\odot}$, and it generally obtains an image set every $12 \mathrm{~s}$. In 2011, SDO made the first positive EUV detections of comets in the solar corona, observing the sungrazers C/2011 N3 SOHO (Schrijver et al. 2012) and C/2011 W3 Lovejoy (McCauley et al. 2013; Downs et al. 2013; Raymond et al. 2014). The light detected arose mainly from O III through O VI ions as they progressed towards the coronal ionisation state of O VII and serendipitously emitted in the EUV channels (Bryans and Pesnell 2012; Pesnell and Bryans 2014). The observations were used both to determine the outgassing rate and composition of the comet, and to study the coronal magnetic field and density structure (Downs et al. 2013).

Operating on the twin STEREO observatories are the EUVI instruments with fields of view extending to $1.7 \mathrm{R}_{\odot}$. This extended view of the corona assisted in EUV observations of C/2011 W3 Lovejoy at $171 \AA$ from vantage points about $107^{\circ}$ ahead of and behind Earth in its orbit (Downs et al. 2013). Observations of Comet Lovejoy were only made possible due to the advanced knowledge of the comet's passage through the field of view, allowing project scientists to prepare observing sequences and sub-field exposures at sufficient cadences to 
Fig. 10 Combined

$\mathrm{SOHO}$-LASCO C2 and

SOHO-UVCS observations of Comet C/2002 X5

Kudo-Fujikawa during 2003

January 27-29. The comet was at

$\sim 1.19$ AU from the spacecraft,

0.19 AU beyond the Sun, and reached perihelion on January 29. In the two UVCS scans, which bracket a single LASCO C2 exposure at January 28 12:12 UT, the blue region shows Ly- $\alpha$ emission, whilst the red tail is composed of $\mathrm{C}^{2+}$ ions (CIII). A disconnection event in the ion tail is observed on January 27 (Povich et al. 2003). The image of the Sun is from the $\mathrm{SOHO}$ Extreme ultraviolet Imaging Telescope, EIT. Composite image courtesy of M.S. Povich

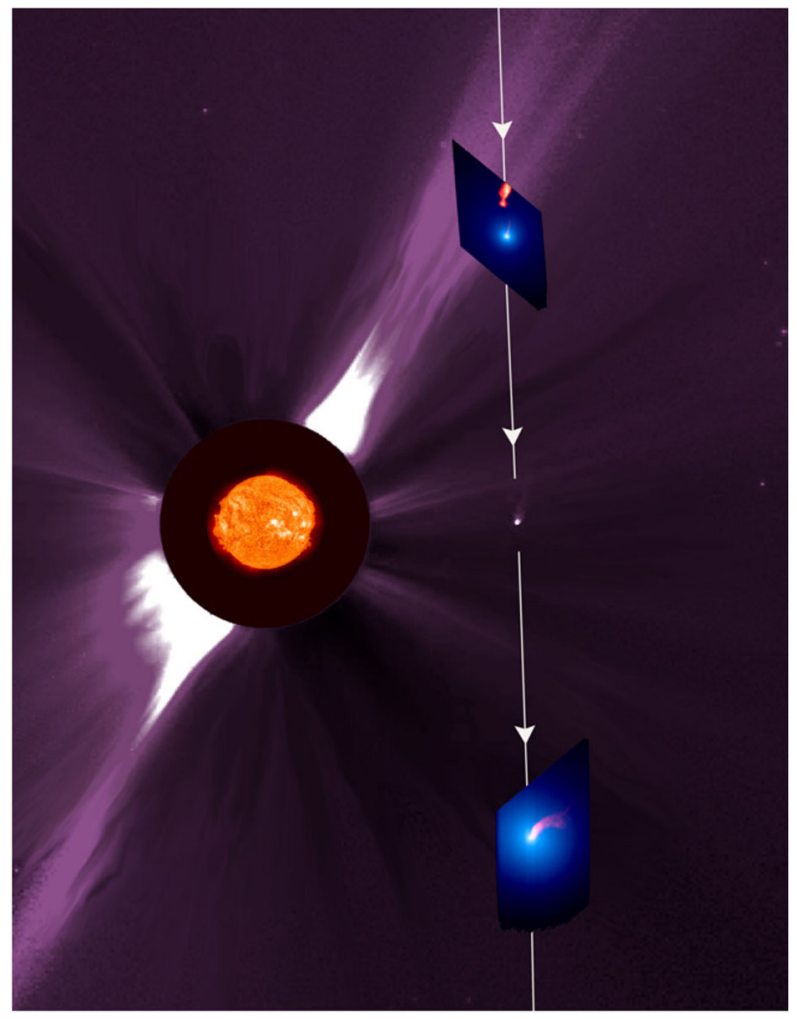

detect the comet. C/2011 N3 SOHO was not visible in these imagers, as its passage was not anticipated and no such observing sequences were prepared.

On 2013 November 28, the Solar Ultraviolet Measurements of Emitted Radiation, SUMER instrument aboard SOHO (Wilhelm et al. 1995) observed C/2012 S1 ISON at ultraviolet wavelengths, shortly after the object's break-up (Curdt et al. 2014). This was the only known observation of a comet by this instrument.

\subsection{Other Space-Based Facilities}

A number of comets have been observed at X-ray wavelengths. These energetic photon emissions are due to charge transfer between cometary neutrals and highly ionised solar wind species (Cravens 2002; Lisse et al. 2004), but only one sungrazer, C/2011 W3 Lovejoy, has been observed in X-rays produced through direct excitation of cometary material. It was seen with the X-ray Telescope on the Hinode spacecraft (Golub et al. 2007). That instrument has $2^{\prime \prime}$ pixels, and the emission was detected only for its thinnest filter, sensitive to the lowest energies. The emission morphology and instrument response indicate that X-rays are produced by excitation of O VII $(\sim 22 \AA)$ after oxygen from the comet reaches a coronal ionisation state (McCauley et al. 2013).

\subsection{Ground-Based Observations}

All historical observations of sungrazing comets were, of necessity, conducted from the ground with the naked eye or telescopically in the visible bandpass. Since 1970, only a 
handful of exceptional sungrazing comets have been observed with traditional Earth-based optical/near-IR telescopes. As previously mentioned, C/2012 S1 ISON was observed extensively through perihelion at many wavelengths. C/2011 W3 Lovejoy was observed by numerous amateurs and a handful of professionals both before and after perihelion, although no ground-based post-perihelion observations detected a central condensation or any other indication of ongoing activity (cf. Sekanina and Chodas 2012; Knight et al. 2012). The remnants of sunskirting Comet C/2015 D1 SOHO were imaged by several observers but no evidence was seen for activity (e.g., Hui et al. 2015; Masek et al. 2015).

Other than C/2011 W3 Lovejoy and C/2012 S1 ISON, no recent sungrazers have been discovered prior to reaching the fields of view of space-based solar observatories. Many observers likely conduct informal searches for potential Kreutz comets, but the most comprehensive published survey was conducted by Ye et al. (2014). That group used MegaCam on the Canada-France-Hawaii Telescope to search for Kreutz comets approximately 1 month before they would reach perihelion, but found none down to a limiting $r^{\prime}$ magnitude of +21 to +22 . These non-detections suggest that either the orbital uncertainty is larger than previously thought or that Kreutz fragments brighten more steeply than other comets. A similar, unsuccessful survey using the Mayall 4-m telescope at Kitt Peak National Observatory was reported by Knight et al. (2010), and other attempts, e.g., a survey with the 0.6-m Curtis Schmidt Telescope at Cerro Tololo Inter-American Observatory in 1991 (T. Farnham, private communication 2015), have likely gone unreported owing to lack of success.

While traditional optical/near-IR telescopes are limited by how close to the Sun they can point, some other telescopes are capable of observing near-Sun comets at very small solar elongations. Sub-mm observations of C/2012 S1 ISON were made with the James Clerk Maxwell Telescope (JCMT) within $\sim 1$ day of perihelion (Keane et al. 2016). Target of opportunity observations by M. Drahus and colleagues to detect bright sungrazers discovered in $\mathrm{SOHO}$ images were triggered on several occasions with the Institut de Radioastronomie Millimetrique (IRAM) 30-m and JCMT. The only comet successfully observed was C/2011 W3 Lovejoy, where HCN was weakly detected by IRAM and not detected by JCMT (M. Drahus, private comm., 2012). Given that Lovejoy was significantly brighter than any $\mathrm{SOHO}$-discovered comet, the target of opportunity program has now been discontinued.

Ground-based solar telescopes have likewise had limited success at observing sungrazing comets. While C/1965 S1 Ikeya-Seki was observed successfully by numerous solar telescopes in 1965 (Becklin and Westphal 1966; Curtis and The Sacramento Peak Observatory Staff 1966; Thackeray et al. 1966; Evans and McKim Malville 1967; Preston 1967; Slaughter 1969), similar attempts to obtain spectroscopy of C/2012 S1 ISON by at least two groups were unsuccessful in 2013: Morgenthaler and colleagues used the National Solar Observatory (NSO)'s McMath-Pierce Solar Telescope on Kitt Peak, and Wooden and co-workers used NSO's Dunn Solar Telescope on Sacramento Peak (Wooden et al. 2013). C/2012 S1 ISON was successfully imaged with a ground-based coronagraph, the Mees Observatory on the summit of Haleakala, Maui, HI, USA, 27 minutes after perihelion (Druckmüller et al. 2014). St. Cyr and Altrock (1993) found no evidence of any of the SOLWIND/SMM discovered comets from 1979-1989 in archival Fischer-Smartt Emission Line Coronal Photometer data from NSO's Sacramento Peak. St. Cyr (private comm., 2015), also looked for the SOLWIND/SMM comets and bright SOHO-discovered Kreutz comets in Mauna Loa Solar Observatory MK4 images without finding any.

There have been numerous likely near-Sun comet discoveries during solar eclipses throughout history (e.g., England 2002; Strom 2002; Kronk 1999, 2003, 2007; Kronk and Meyer 2010). Two prominent examples include suspected Kreutz Comet X/1882 K1 Tewfik which was only seen during the 1882 total solar eclipse (Fig. 11), and C/1948 V1 
Fig. 11 Photograph of suspected Kreutz sungrazer X/1882 K1 Tewfik (lower right), obtained in Egypt during the 1882 May 17 solar eclipse (Abney and Schuster 1884). Image courtesy of the Royal Astronomical Society

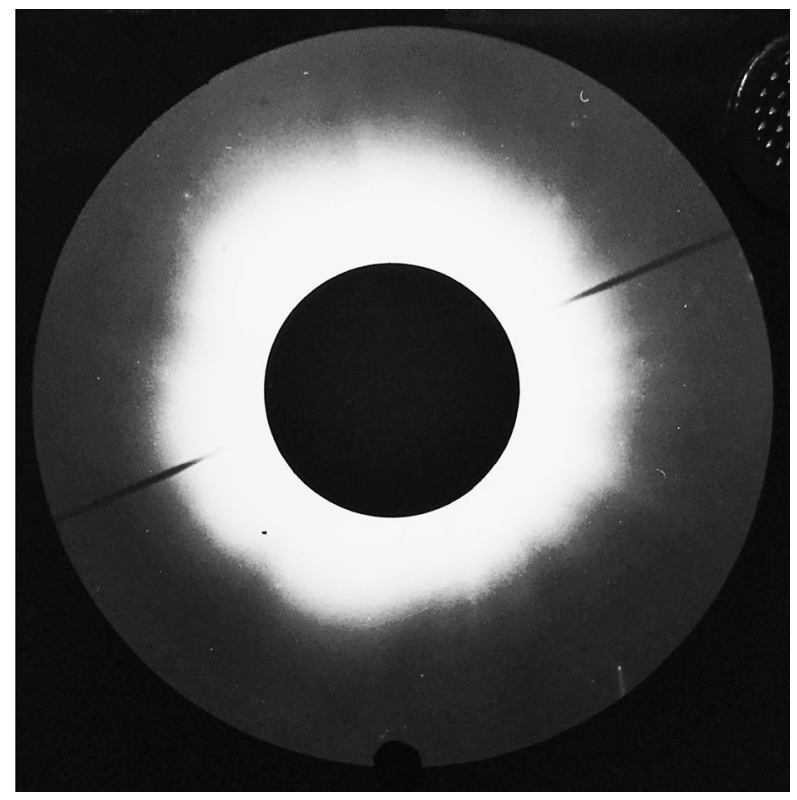

Table 4 Typical orbital parameters for known groups of sungrazing and sunskirting comets. Note that the Marsden and Kracht groups contain numerous comets that are suspected to have been observed more than once. The total number of unique comets for those groups is likely to be smaller than this number. The number of comets is current as of 2017 October

\begin{tabular}{llrlclr}
\hline Group & $q\left(\mathrm{R}_{\odot}\right)$ & $i\left(^{\circ}\right)$ & $\omega\left(^{\circ}\right)$ & $\Omega\left({ }^{\circ}\right)$ & $\mathrm{P}(\mathrm{yr})$ & Number \\
\hline Kreutz & $1-2$ & 143 & 80 & 0 & $500-1000$ & 2940 \\
Marsden & $10-12$ & 27 & 24 & 79 & $5.3-6.1$ & 54 \\
Kracht & $9-11$ & 13 & 59 & 44 & $4.8-5.8$ & 42 \\
Meyer & 8 & 73 & 57 & 73 & Unknown & 220 \\
\hline
\end{tabular}

$\left(q=0.135 \mathrm{AU} ; 29.0 \mathrm{R}_{\odot}\right)$ which was subsequently followed for $\sim 5$ months. Despite the tremendous improvement of telescopic and photographic capabilities in modern times, the only definitive modern detection of a sungrazing comet during an eclipse was C/2008 O1 SOHO by Pasachoff et al. (2009).

\section{Populations}

\subsection{Near-Sun Cometary Groups}

As previously discussed, the vast majority of near-Sun comets are members of groups of dynamically related objects: Kreutz, Marsden, Kracht, or Meyer. All comets in a particular group are believed to have ultimately descended from a single progenitor comet which has undergone repeated fragmentation/disruption events to produce the members known today. Typical orbital elements of the groups as well as the number of known members are given in Table 4. We now discuss each group in more detail, together with their possible origins. 

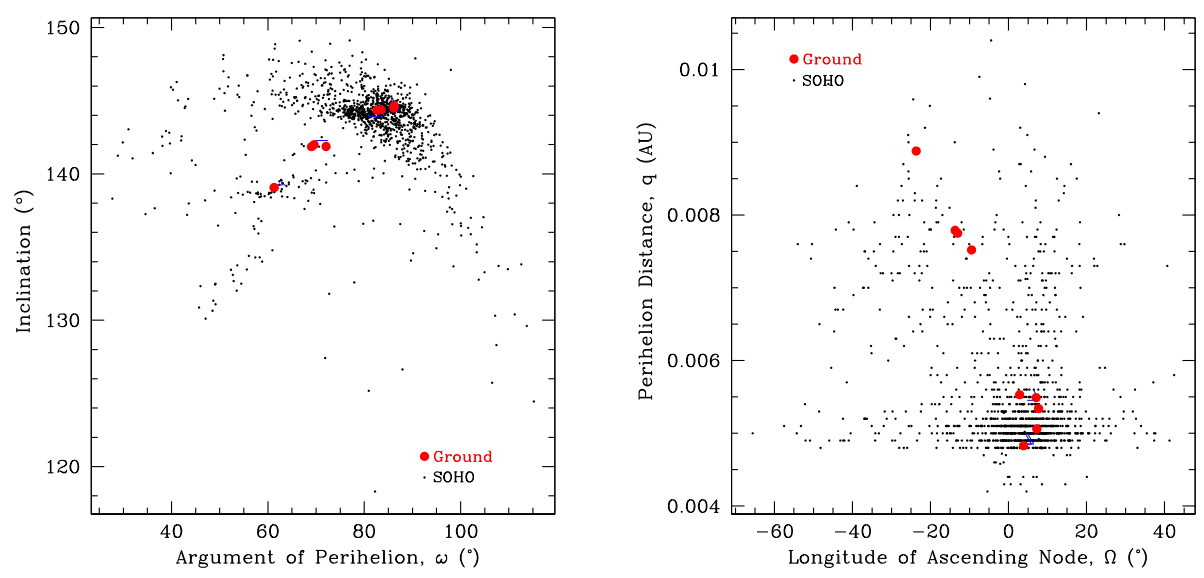

Fig. 12 Orbital elements of the Kreutz group. The left panel plots the inclination $(i)$ against argument of perihelion $(\omega)$. The right panel plots the perihelion distance $(q)$ against longitude of the ascending node $(\Omega)$. The red circles are comets observed from the ground up to 1970. The black crosses are all SOHO observed comets discovered to May 2008. The orbital elements for the $\mathrm{SOHO}$ comets were compiled from Minor Planet Electronic Circulars (MPECs) and International Astronomical Union Circulars (IAUCs). After Knight (2008)

\subsubsection{Kreutz Group}

Originally recognized by the similarity of the orbits of several prominent sungrazers in the 1800s (Kirkwood 1880; Kreutz 1888, 1891, 1901), the Kreutz group had long been the only definitive association of dynamically related comets (e.g., Boehnhardt 2004). The distribution of the Kreutz group members' orbital elements is shown in Fig. 12. Interest in the groups' dynamical history was rekindled by the spectacular appearance of three bright members between 1963 and 1970 (e.g., Öpik 1963, 1966; Kresák 1966; Marsden 1967; Sekanina 1967). The SOLWIND and SMM discoveries, followed soon after by $S O H O$ and eventually STEREO, have yielded extensive investigations into the group's hierarchy and the processes driving its creation (e.g., Weissman 1983; Marsden 1989; Sekanina 2002b).

Whilst there is no consensus about the specific fragmentation history of the Kreutz group, most investigators (Marsden 1967, 1989; Sekanina and Chodas 2002a,b, 2004, 2007, 2008) are in agreement about the general picture of the group's evolution. At some point, likely in the last several thousand years, the group's original parent comet was perturbed into a sungrazing orbit and broke up near perihelion. It has been suggested that the comet of 372 BCE, alleged to have been observed by the Greek historian Ephorus to split near the Sun, was the parent comet, but this linkage is speculative at best (Marsden 1967). The sibling fragments next reached perihelion hundreds of years later, with fragments potentially separated in time by decades or possibly centuries. Large sibling fragments likely split again near perihelion, while smaller sibling fragments were destroyed. The grandchild fragments again reached perihelion hundreds of years later and temporally separated by decades to centuries. This process continued to the present day, with each generation of large comets splitting into a new generation, and the small comets being destroyed on their next perihelion passage. Because the changes in orbital period are on the order of decades, this repeated process may lead to later generations being mixed temporally, e.g., higher generation fragments on shorter orbits may reach perihelion earlier than longer period, lower generation fragments. Based on the similarity of some $\mathrm{SOHO}$-observed Kreutz orbits, Sekanina (2000, 
2002a) proposed that this cascading fragmentation can occur throughout the orbit and is not confined to near perihelion.

The small Kreutz comets observed regularly by SOHO and STEREO have presumably been produced at their previous perihelion passage as parts of larger bodies. Otherwise, comets are far too small to survive perihelion passage. Knight et al. (2010) showed that if the distribution of small Kreutz comets seen by SOHO during 1996-2005 is representative of the distribution all the way around the Kreutz orbit, then the total mass of all small Kreutz comets is likely smaller than a single $\sim \mathrm{km}$ scale nucleus. At least four of the brightest Kreutz comets of the last 200 years (C/1843 D1, C/1882 R1, C/1963 R1 Pereyra, C/1965 S1 Ikeya-Seki) were likely larger than a $\mathrm{km}$ in radius, suggesting that the majority of the mass of the system remains locked in the largest few fragments and that the Kreutz system is still evolutionarily young (Sekanina 2002b; Knight et al. 2010). Despite the many spectacular apparitions of Kreutz comets throughout history, the original progenitor need not have been exceptionally large; a comet a few $\mathrm{km}$ to tens of $\mathrm{km}$ in radius (e.g., C/1995 O1 Hale-Bopp sized or smaller; Weaver et al. 1997) could have produced the entire known Kreutz group.

The morphologies of SOHO and STEREO observed Kreutz members are diverse. In all coronagraph data, they vary from small and stellar, only 1-2 pixels across, to broad and diffuse, extending over several pixels. Only a few exhibit tails, and these again vary from narrow and thin to broad and diffuse, extending from a few arcminutes up to a degree or more. Brighter objects are more likely to exhibit tails but there does not appear to be a clear correlation between brightness, morphology, and length of visible tails (Battams and Knight 2016).

The clustered nature of Kreutz comet returns has been noted both for ground-based observations (Marsden 1967), and for coronagraphic discoveries (MacQueen and St. Cyr 1991; Sekanina 2000; Knight 2008). These objects will frequently arrive in close pairs, or with several objects over a period of a few days, prior to or following an apparent lull in arrivals. Again there is seemingly no correlation in the morphology of clustered Kreutz fragments, with large, diffuse objects frequently appearing alongside small, quasi-stellar counterparts. If these fragments were part of the same object in the relatively recent past, the diverse morphologies may be indicative of the non-uniform composition of their progenitor.

The Kozai resonance (Lidov 1962; Kozai 1962) can cause objects to exchange angular momentum between the eccentricity and the inclination of their orbits, and works well for objects in the planetary system such as asteroids and Jupiter family comets, JFCs. It may not work as well for comets such as the Kreutz group which have semi-major axes $>50 \mathrm{AU}$. Also, the Kozai resonance reduces the perihelion distance gradually, by a limited amount each orbit. Thus the Kreutz progenitor may have begun fragmenting, e.g. due to thermal stresses, before it passed within the solar Roche limit, and we would see that history in the observed Kreutz population if that were the case.

The more likely origin of the Kreutz group parent is a long-period comet from the Oort Cloud whose path was transformed by stellar perturbations and galactic tides into an orbit with near-zero angular momentum. Although this process is believe to be rare, it is not impossible. Everhart (1967)'s estimate of the perihelion distribution of long-period comets, corrected for observational selection effects, shows that a substantial number of long-period comets are still expected to pass at very small perihelion distances.

A problem with the current Kreutz group comets for which the semimajor axes have been determined is that their orbits are all well detached from the Oort Cloud, with aphelia $<200$ AU. This is despite the fact that the Kreutz group orbit is oriented so that its members cannot closely approach any planet. Weissman (1980) proposed that very strong non-gravitational forces from the jetting of nucleus surface volatiles could have changed the 
parent comet's semimajor axis to the currently observed values in only 2 or 3 returns. Also, dynamical modeling of the tidal breakup of cometary nuclei in sungrazing orbits (Weissman et al. 2012) has shown that the parent comet and its subsequent fragments have likely gone through two, three, or more returns to obtain the current spread in arrival times, as discussed in Sect. 7.1.

\subsubsection{Marsden and Kracht Groups}

The Marsden and Kracht families are the best studied associations of comets after the Kreutz group. Neither group's existence was known prior to the launch of $\mathrm{SOHO}$, and no members of either group have ever been observed by any ground-based telescope. A small number of these, such as C/2008 R7 (Su et al. 2008), have been observed by either STEREO spacecraft. The groups were recognized based on the similarity of the trajectories of a handful of comets in SOHO images (Kracht et al. 2002b; Marsden and Meyer 2002). Both groups are sunskirting, with perihelion distances nearly an order of magnitude larger than the Kreutz group. As a result, many are observed to survive perihelion, and tentative linkages between comets have been proposed with orbital periods of 5-6 years (see Table 4.1 in Knight 2008 and references therein). Once potential linkages were identified and orbital paths projected, additional members were subsequently found in archival data. All members of each group brighter than the $\mathrm{SOHO}$-LASCO detection limits have likely now been catalogued.

While the Marsden and Kracht orbits are currently dissimilar (as shown in Table 4), backwards orbital integrations strongly suggest a common origin. Studies by Ohtsuka et al. (2003) and Sekanina and Chodas (2005) have shown that these two groups are likely related to Comet 96P/Machholz 1 as part of the "Machholz Complex". This association also includes several meteor streams, first noted by D.A.J. Seargent (Kracht et al. 2002a), the asteroidal object $2003 \mathrm{EH} 1$, and possibly Comet C/1490 Y1. The latter linkage is, however, disputed (Micheli et al. 2008). These authors argue that the progenitor of the Machholz Complex likely split prior to $950 \mathrm{CE}$, and the orbits of subpopulations likely evolved at different rates due to small variations in the timing of interactions with Jupiter. Earlier researchers (Rickman and Froeschle 1988; Green et al. 1990; Bailey et al. 1992) had noted that 96P's orbit could become sungrazing in the future, but the discovery of small- $q$ objects dynamically related to it was surprising nonetheless.

Initial linkages between members of each group were made based on their orbital elements. However, the orbital arcs are generally too short to be definitive, so Knight (2008) and Lamy et al. (2013) used the comets' SOHO lightcurves to establish the most likely linkages. The best observed comets in these groups do not appear to have faded significantly, suggesting that they are large enough that the mass lost during each orbit is not a substantial fraction of the total nucleus mass. This argument suggests they are likely considerably bigger than typical $\mathrm{SOHO}$-observed Kreutz comets, but no plausible size estimates have been published.

There is some evidence that the Marsden and Kracht populations are not in a steady state. The known members are highly temporally clustered, with several fragments often arriving within a few days of each other followed by stretches of several months without any detections (see Fig. 12 of Lamy et al. 2013). Knight (2008) proposed tentative fragmentation hierarchies of each group that could trace all known members into just a few discrete objects several orbits earlier. Dynamical simulations by the same author suggested the entire distribution of each group could have been produced by low velocity fragmentations of a single object over the last several hundred years. It appears that the frequency of arrivals detected by $\mathrm{SOHO}$ has decreased over time, with the faintest comets failing to be recovered. 
This may be indicative of the comets losing their volatiles and/or eroding substantially from one apparition to the next. However, the statistics are sparse and many Marsden and Kracht comets are near the detection thresholds for $\mathrm{SOHO}$ and could therefore be missed due to poor viewing geometry or data gaps on a subsequent passage.

The likely origin of the parent of the Machholz Complex is the Scattered Disk population (Levison and Duncan 1997), which are distant comets with perihelia close to Neptune's orbit. Gravitational interactions with the giant planets drive these comets to become JFCs over $\sim 10$ Myr timescales (Levison and Duncan 1997). Once in the JFC population, the Kozai resonance can cause some of them to exchange angular momentum between the eccentricity and the inclination of the orbit. Angular momentum itself is conserved. The result is that some orbits can be driven to very small perihelia where they are observed as sunskirting comets.

\subsubsection{Meyer Group}

The Meyer group is the second most populous group of near-Sun comets and, like the Marsden and Kracht groups, was unknown prior to the launch of SOHO (Marsden and Meyer 2002). As of 2017 October, there are 220 apparent members of this group. There have been no proposed linkages between Meyer group comets, nor have they been dynamically linked to any other solar system objects. As a result, their orbits are based entirely on the short ( $\lesssim 2$ days), low resolution arcs in $\mathrm{SOHO}$ images and their orbital periods are not constrained. Marsden (personal comm.) noted the high inclinations and lack of clustering in the Meyer group arrivals (see Fig. 12 of Lamy et al. 2013) and suggested that the group likely had a long orbital period of at least decades, most likely centuries, and was already evolutionarily evolved, i.e., there is little ongoing fragmentation.

Meyer group comets are sunskirters, having perihelia slightly closer to the Sun than the Marsden and Kracht groups, but substantially farther than the Kreutz group. Many members are observed post-perihelion so it is assumed that they are not destroyed and will return on subsequent orbits. Typical Meyer comets do not exhibit an obvious coma or tail, so their designation as comets is based primarily on their high inclination comet-like orbits. Most are near the detection threshold of $\mathrm{SOHO}$ (Lamy et al. 2013), and comparably faint Kreutz, Marsden, and Kracht objects, all of which are dynamically related to known comets, have similar non-cometary appearances. Thus, a cometary origin cannot be ruled out. Assuming that the Meyer comets are dynamically mature and have reached comparably small heliocentric distances repeatedly, they may be almost entirely devoid of volatiles and only active under the extreme conditions near the Sun. Battams and Knight (2016) argued that the group's progenitor need not have been larger than a moderately sized JFC nucleus.

The origin of the Meyer group comets is uncertain, but this group's high inclination suggests that its progenitor was a dynamically evolved Oort Cloud comet, similar to a HalleyType Comet.

\subsubsection{Other Near-Sun Comets}

As of 2017 October, 149 comets have been discovered in SOHO and, occasionally, STEREO images that do not belong to any of the groups discussed above. A small number of these "sporadic" or "non-group" objects are comets with larger perihelion distances that serendipitously passed through the $\mathrm{SOHO}$ field of view (e.g., P/2003 T12 SOHO = $2012 \mathrm{~A} 3$; Hui 2013), but the majority are sunskirting or sungrazing. Most are sparsely observed with poorly determined orbits that are not obviously linked to any other known objects. Finally, 
for sporadic sungrazers, the Oort Cloud is the likely origin because the inclinations of these objects are randomly scattered across the sky. Only a relatively small number of objects are known with long, comet-like orbital periods that are apparently asteroidal ("Damocloid", like 1996 PW, e.g., Weissman and Levison 1997) or weakly active ("Manx comet"; Meech et al. 2016). Such objects may represent the first stages in the development of future sunskirting groups.

The majority of sunskirting and sungrazing non-group comets appear as small, stellar objects with no visible tail or coma, though a minority exhibit one or both of these phenomena. Occasionally, non-group comets appear as close pairs, separated by minutes to hours. Presumably these are objects that fragmented a significant time earlier, as the spatial resolution of the LASCO instruments are such that the physical distance between fragments must be substantial, and separation velocities necessary to create sufficient separation would be nonphysical over short timescales.

Due to the poor quality of orbit determinations from $\mathrm{SOHO}$ data, it is possible that some of these non-group comets may be repeated apparitions of the same object. For example, non-group Comet C/1999 X3 SOHO = 2004 E2 = 2008 K10 (Kracht and Marsden 2008) was identified in 2008 as a single object with a roughly 4.2-year orbital period and is now designated 323P/SOHO 2. Little information can be gleaned from such objects besides their lightcurve behavior (shown for most "sporadic" objects in Lamy et al. 2013), but we discuss the three most interesting objects below.

Sunskirter 322P/SOHO $1=1999 \mathrm{R} 1 \mathrm{SOHO}=2003 \mathrm{R} 5=2007 \mathrm{R} 5=2011 \mathrm{R} 4$ has $q=$ $0.057 \mathrm{AU}\left(12.26 \mathrm{R}_{\odot}\right)$, a 3.99 year period, and has been definitively seen on five apparitions (it was not given a unique designation until 2015). The linkage was initially recognized by R. Kracht (Hammer et al. 2002) and subsequent returns were accurately predicted by Hönig (2006). Knight and Battams (2007) and Lamy et al. (2013) found that the lightcurve was virtually identical at each apparition. While 322P has not exhibited a tail or obvious coma, its lightcurve is inconsistent with a bare asteroid (Knight and Battams 2007). Hönig (2006) could not link it to any known solar system object. Though its Tisserand parameter of 2.3 (Knight et al. 2016) suggests that it is of cometary origin, Hönig also, noted that its current orbit is near the 3:1 resonance with Jupiter, making it difficult to explore its longterm dynamical history.

Knight et al. (2016) observed 322P at $>1$ AU from the Sun with ground-based optical telescopes and Spitzer, finding that it was inactive with a high albedo (0.09-0.42), implying that it is $150-320 \mathrm{~m}$ in diameter. They also found it had unusual colors for a comet nucleus and inferred a density $>1000 \mathrm{~kg} \mathrm{~m}^{-3}$ if it was a strengthless body. They concluded that $322 \mathrm{P}$ may be asteroidal in origin and only active in the $\mathrm{SOHO}$ fields of view due to nonvolatile driven activity (see the following sub-section). Currently, 322P is one of only two periodic near-Sun comets observed from the ground (96P/Machholz 1 is the other).

Three poorly observed objects in orbits similar to 322P have been discovered in $\mathrm{SOHO}$ images, C/2002 R5, C/2008 L6, and C/2008 L7, with the collection sometimes referred to as the "Kracht II group." Note that this group is not in any manner dynamically related to the Kracht group; both were first recognized by R. Kracht. Kracht and Sekanina (Kracht et al. 2008) proposed that C/2002 R5 split into the latter two, but none were observed at what would have been their next return in 2014 so the linkage remains uncertain.

C/2015 D1 SOHO was by far the brightest non-Kreutz comet discovered by $S O H O$, peaking at a $\mathrm{V}$ magnitude of $\sim 1.3$ (Hui et al. 2015). The sunskirter $\left(q=0.028 \mathrm{AU} ; 6.02 \mathrm{R}_{\odot}\right)$ developed a well-defined tail in post-perihelion $\mathrm{SOHO}$ images and appeared as a tail of dust 
lacking any central condensation when recovered from the ground by amateur observers a few days later (Masek et al. 2015). Orbital calculations based on the SOHO images required either separate pre- and post-perihelion solutions (Williams 2015) or strong nongravitational forces (Hui et al. 2015). Taking all of these factors into account, it appears that C/2015 D1 disrupted at or near perihelion. Its orbit does not appear to be related to any other known solar system object. Its high inclination $\left(\sim 70^{\circ}\right)$ suggests a long period or Oort Cloud origin, but the orbit is insufficiently constrained to determine whether it had previously passed so close to the Sun (Hui et al. 2015).

\subsection{Near-Sun Asteroids}

While all of the objects discovered in SOHO and STEREO images have been termed "comets," it is not definitively known that they are all of classically cometary origin, e.g., active due to sublimation of volatile ices. It is likely that the Kreutz group members are cometary in nature, as some large members of that association, such as C/1965 S1 IkeyaSeki, were clearly comets when discovered pre-perihelion at larger heliocentric distances.

The Meyer, Kracht, and Marsden comets, as well as the majority of objects with no group identification, appear as entirely stellar objects in the SOHO-LASCO and STEREOSECCHI fields of view. It is only from observation of their comet-like lightcurves during their perihelion passages that these objects are tentatively classified as comets. Objects in the Meyer, Kracht and Marsden groups may not display visible comae or tails because they have largely been devolatilized at repeated prior passages close to the Sun. Inactive asteroidal objects, e.g., bare nuclei, would need to be $\geq 10 \mathrm{~km}$ in diameter to be visible in $\mathrm{SOHO}$ or STEREO images. Such objects would be unlikely to have been missed at larger heliocentric distances by traditional surveys. Thus, it is very likely that all objects, whether of traditional cometary or asteroidal origin, have a dust coma present when observed by SOHO and STEREO. Such a dust coma could plausibly be produced from a canonically asteroidal object. As noted in Sect. 9.1, refractory materials will begin sublimating at these distances (e.g., Kimura et al. 2002). Jewitt and Li (2010) and Jewitt (2012) have shown that thermal decomposition and thermal fracture can plausibly produce detectable quantities of dust, hence Jewitt et al. (2015) terms such objects "active asteroids."

In the absence of observations of cometary activity at larger heliocentric distances, a dynamical link with known comets would be needed to demonstrate that a particular $\mathrm{SOHO}$ discovered object is canonically cometary in origin. Even then, such an object may be devoid of accessible volatile ices due to evolutionary effects.

Low- $q$ asteroids have been predicted to exist (e.g., Farinella et al. 1994; Gladman et al. 1997; Bottke et al. 2002; Greenstreet et al. 2012), but Granvik et al. (2016) argue that they are destroyed quickly due to catastrophic disruption. As of 2017 October, JPL Horizons lists 39 asteroids with perihelia within the sunskirter region of $<0.15 \mathrm{AU}\left(33.1 \mathrm{R}_{\odot}\right)$. Subsets of these have been reviewed by Campins et al. (2009) and Jewitt (2013), although in both cases the authors generally considered objects with $q$ significantly beyond $0.15 \mathrm{AU}$, so the results may not be applicable to objects observed by SOHO and/or STEREO.

Only one asteroid with $q<0.15$ AU has been detected by solar observatories, 3200 Phaethon. Phaethon was discovered in 1983 and was classified as an asteroid (Green and Kowal 1983). It was immediately recognized that its orbit was very similar to that of the Geminid meteoroid stream (Whipple 1983). Recently, Phaethon has exhibited a faint but active dust coma in STEREO-SECCHI-HI1 images (Jewitt and Li 2010; Jewitt 2013; Li and Jewitt 2013; Hui and Li 2017), although the activity was insufficient to support the Geminids. There is some question as to whether or not Phaethon could have retained volatiles. 
Jewitt and Li (2010) argued that its blackbody temperature was too high for buried ices to survive. Conversely, Boice (2017) finds that primitive volatiles can be preserved in its interior due to the very low thermal conductivity typical of small solar system bodies despite repeated low perihelion passages. Phaethon is the target of the planned JAXA DESTINY+ mission.

\subsection{Vulcanoids}

A population of near-Sun asteroids residing on stable orbits entirely within the orbit of Mercury has long been postulated and is known as the "Vulcanoids" after the proposed planet interior to Mercury (Le Verrier 1859). Vulcanoids smaller than $1 \mathrm{~km}$ in diameter are removed from the region by the Yarkovsky effect in less than the lifetime of the solar system (Vokrouhlický et al. 2000), while objects smaller than $\sim 70 \mathrm{~m}$ would have accreted onto the Sun through Poynting-Robertson drag (Schumacher and Gay 2001). Larger objects could be on stable orbits but are depleted from the region by collisions (Leake et al. 1987). Numerous searches for Vulcanoids have been conducted over the years (e.g., Perrine 1902; Campbell and Trumpler 1923; Leake et al. 1987), but none have ever been found. Systematic searches of the SOHO-LASCO (Durda et al. 2000) and STEREO-SECCHI (Steffl et al. 2013) datasets leave only a small size range for any possible Vulcanoids: $1.0-5.7 \mathrm{~km}$ in diameter. Steffl et al. (2013) conclude that any current population of Vulcanoids would be the collisionally processed remnants of a primordial population which now contains at most 76 objects larger than $1 \mathrm{~km}$ in diameter.

\section{Lightcurves}

\subsection{Introduction}

In the absence of a coma, the individual nuclei of most near-Sun comets would still be far below the detection threshold of SOHO-LASCO and STEREO-SECCHI. It is the presence of a coma that allows most sungrazers to be observable, but even then, most of their comae are not resolved, due to these instruments' large angular pixel sizes. Traditional nucleus and coma studies therefore cannot be conducted. Instead, information about most near-Sun comets is gleaned by conducting aperture photometry of the coma. Brightness measurements are typically plotted as a function of time or heliocentric distance for the whole apparition (the "secular lightcurve") since the large pixels suppress short-term variations, generally precluding the detection of a rotational lightcurve.

Most sungrazing comets are observed for hours to days, which is generally too brief for detailed study. Thus, the lightcurves of large numbers of comets have been studied in aggregate to yield properties of the Kreutz group (Biesecker et al. 2002; Knight et al. 2010), the Marsden, Kracht, and Meyer groups, as well as "sporadic" comets (Lamy et al. 2013).

\subsection{Lightcurve Profiles}

The slope of brightening or fading in a secular lightcurve can reveal information about the processes at work at the nucleus or in the coma. Comets observed at typical heliocentric distances $\left(r_{\mathrm{H}}\right)$ near and beyond 1 AU canonically brighten $\propto r_{\mathrm{H}}^{-4}$, although slopes ranging from $r_{\mathrm{H}}^{-1}$ to $r_{\mathrm{H}}^{-10}$ are not uncommon (e.g., A'Hearn et al. 1995). Knight et al. (2010) found that Kreutz comets initially brighten very rapidly when entering the $\mathrm{SOHO}$ fields of view $\left(r_{\mathrm{H}}^{-7.3}\right)$, 
then close to the canonical rate of $r_{\mathrm{H}}^{-4}$ until they reach peak brightness and begin to fade. It is unknown how far out the steep rate of brightening extends, but Ye et al. (2014) found it likely begins beyond $50 \mathrm{R}_{\odot}(0.232 \mathrm{AU})$. This is also seen qualitatively, as many comets appear to brighten very rapidly when first detected in SOHO-LASCO or STEREO-SECCHI images (Battams and Knight 2016). The brightening or fading rates of most non-Kreutz comets observed by SOHO or STEREO are not well understood because these comets are generally fainter and are not observed over a large enough range of heliocentric distances.

Such a steep rate of brightening suggests that these comets' activity is not being controlled by $\mathrm{H}_{2} \mathrm{O}$ sublimation since that would be expected to proceed near the canonical $r_{\mathrm{H}}^{-4}$. Therefore, most of the small Kreutz objects may have been heavily depleted of water and other volatiles when they split from their parent fragment on a previous apparition. Notable exceptions to this are C/2012 E2 SWAN, the only Kreutz comet to be detected by SOHO-SWAN, which brightened near $r_{\mathrm{H}}^{-4}$ (Ye et al. 2014) and several of the historic ground-observed Kreutz (Sekanina 2002b) which survived perihelion and were orders of magnitude larger than typical SOHO-observed Kreutz (e.g., Sekanina 2002b). It is likely that the small Kreutz objects are minimally active or inactive at ordinary heliocentric distances (e.g., beyond $\sim 0.5 \mathrm{AU} ; \sim 107 \mathrm{R}_{\odot}$ ). They likely brighten rapidly when typically non-volatile material(s) begin to sublimate.

The bandpasses in which most SOHO and STEREO images are taken are broad compared to traditional narrowband comet filters (e.g., Farnham et al. 2000). Thus, they are primarily sensitive to dust reflecting the solar continuum and, in the case of SOHO-LASCO's orange and clear filters, the strong Na I doublet at $5889 \AA$ and $5896 \AA$ (Biesecker et al. 2002; Knight et al. 2010). The normal cometary gas emission bands likely contribute a small amount of signal in some filters (see Fig. 9), but are not believed to contribute significantly to the flux except, perhaps, for CN emission in STEREO-SECCHI HI1, and neutral Fe (Fulle et al. 2007) in very active comets (see Fig. 9).

A surprising feature of Kreutz lightcurves is that they peak in brightness at $\sim 10-14 R_{\odot}$ (0.046-0.065 AU) prior to perihelion and fade interior to that despite continuing to approach the Sun (see Fig. 13). This turnover in brightness is believed to be due primarily to the increasing rate of sublimation of dust grains in the coma, notably olivines and pyroxenes which begin sublimating in this temperature range (Kimura et al. 2002). Biesecker et al. (2002) concluded that Kreutz lightcurves peak at two characteristic distances, suggesting two populations with distinct compositional differences. However, Knight et al. (2010) examined a significantly larger sample and concluded that the distribution was not bimodal and represented a continuum of compositions. There does not appear to be a characteristic lightcurve shape or consistent distance of peak brightness for any of the other groups (Lamy et al. 2013).

Inside of $\sim 10 \mathrm{R}_{\odot}(\sim 0.046 \mathrm{AU})$, lightcurves are generally chaotic. Faint comets tend to rapidly disappear but bright Kreutz often fade then exhibit a second brightening that continues until the comet disappears behind the occulting disc (Biesecker et al. 2002; Knight et al. 2010). UVCS observations (Sect. 10.1) suggest that this second brightening corresponds to the activity of the final remnants of the nucleus (Uzzo et al. 2001). Smaller comets have likely been completely destroyed by this point so do not exhibit a second peak. Inside this distance, the lifetimes of virtually all refractory materials are so short that the coma disappears rapidly and the apparent brightness drops precipitously, even if the comet nucleus remains active (Huebner 1970; Marcus 2013b). This explains the disappearance of the tail of C/1965 S1 Ikeya-Seki when closest to perihelion (Huebner 1970) and the rapid postperihelion brightening and tail regrowth of C/2011 W3 Lovejoy (Knight et al. 2012). 
Fig. $13 \mathrm{SOHO}$-LASCO C2/C3 optical lightcurve photometry for a Kreutz group comet, after Knight et al. (2010). The curves of similar comets all tend to peak at $\sim 10-14 \mathrm{R}_{\odot}$

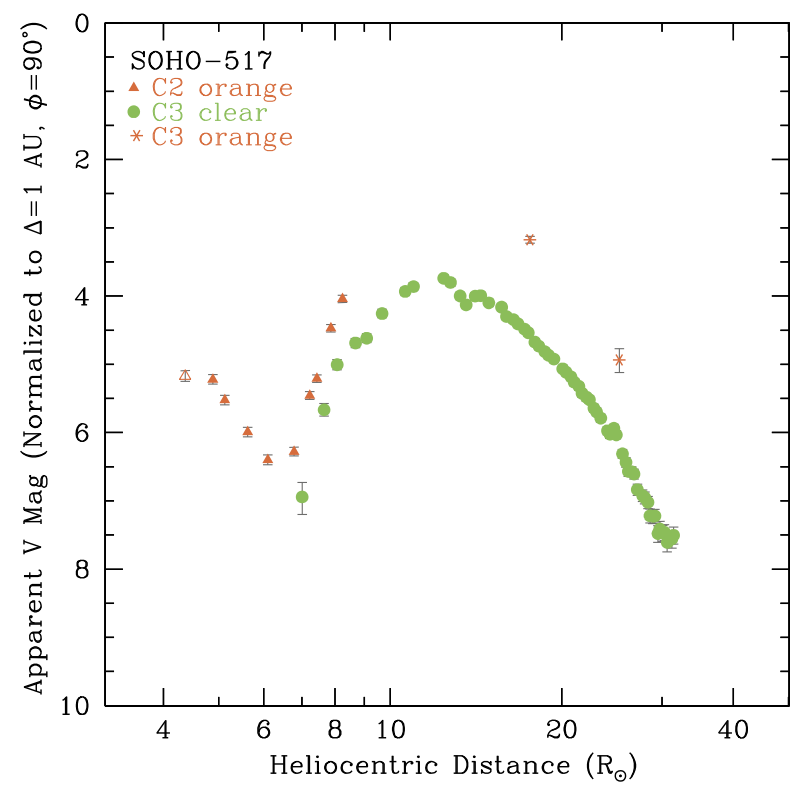

\subsection{Colors}

Color information is relatively limited since STEREO-SECCHI has no movable filters and most observations by $\mathrm{SOHO}$-LASCO are made with the clear filter (C3 telescope) or orange filter (C2 telescope). Other filters (see Table 3 ) are typically acquired daily or less frequently, although special color sequences are occasionally initiated by the $\mathrm{SOHO}$ team when a bright comet is known in advance. The most significant conclusion from the comparison of comets' colors in these images is that all near-Sun comets are significantly brighter in the orange filter than the clear filter, typically by $\sim 1$ magnitude. This is attributed to the very bright $\mathrm{Na} I$ doublet emission which is much stronger relative to the narrow orange bandpass than the wider clear bandpass. It is not an instrumental effect since comets observed at larger heliocentric distances have similar orange and clear magnitudes (e.g., 2P/Encke by Lamy et al. 2003b). Knight et al. (2010) showed that if sodium fluorescence is the only source of the color difference, then sodium emission greatly exceeds the flux from reflected solar continuum in the orange bandpass. They also found that the color difference peaked at $\sim 18.6 R_{\odot}$ $(\sim 0.086 \mathrm{AU})$ and decreased to near zero inside of $10 \mathrm{R}_{\odot}(0.046 \mathrm{AU})$. It has been assumed that the decrease in the orange-clear color inside of $10 \mathrm{R}_{\odot}$ is due to loss of sodium in the coma, but Marcus (priv. comm.) has suggested that it may be due to the increasing thermal radiation from dust grains.

There has been minimal work examining color differences between other filters or between the $S O H O$ and STEREO bandpasses primarily due to sparse simultaneous data. However, Knight and Battams (2014) found that C/2012 S1 ISON had significant color differences pre-perihelion but essentially no color differences post-perihelion. They attribute this to the absence of newly produced material post-perihelion since ISON's nucleus was apparently destroyed prior to perihelion (Sekanina and Kracht 2014). 


\subsection{Effects of Phase Angle}

The viewing geometry of near-Sun comets can change rapidly around perihelion since they traverse a significant true anomaly range very quickly. Furthermore, comets that pass between the observer and the Sun enter a forward-scattering geometry that can enhance their brightness by $>1000 \times$ (Marcus 2007). These changes are exacerbated when comparing data collected from more than one spacecraft (e.g., SOHO, STEREO-A, STEREO-B), since they each have a very different viewing geometry. Thus, lightcurves are generally normalized to a common observer-centric distance and phase angle. Note that the scattering behavior of coma dust is very different to the linear phase correction often used for bare comet nuclei (e.g., Lamy et al. 2014). The most commonly used dust phase correction combines small phase angle corrections from 1P/Halley (Schleicher et al. 1998) with large phase angle corrections from near-Sun comets (Marcus 2007) and is tabulated at the website http://asteroid.lowell.edu/comet/dustphase.html.

Since gas emission is isotropic, the dust phase angle correction should only be applied to the fraction of the signal that is due to dust. Marcus (2007) provides a parameter for this, but the dust-to-gas ratio is rarely known in near-Sun comets so there are unavoidable systematic uncertainties. Hui (2013) found that the forward-scattering enhancement of P/2003 T12 SOHO (which was observed at $r_{\mathrm{H}} \sim 0.6 \mathrm{AU}\left(\sim 129 \mathrm{R}_{\odot}\right)$ so was not a near-Sun comet) greatly exceeded the modeled correction at phase angle $>173^{\circ}$, so extremely large forwardscattering conditions are not yet well modeled but are also exceedingly rare. Such high phase angle measurements can yield unique information about the properties of the dust in the coma, such as the refractive index from Mie scattering modeling (Hui 2013).

\section{Nucleus Parameters}

As mentioned previously, given the limited spatial resolution of coronagraphs, it is not possible to separate the nucleus signal from that of the coma. Some information on the nucleus itself can be derived however from coronagraph data. In addition, scanning, quasiimaging spectroscopic observations with SOHO-UVCS, STEREO-EUVI, and SDO-AIA have yielded nucleus information for a limited number of comets.

\subsection{Sizes}

In general, comet nucleus radii range from $0.3-25 \mathrm{~km}$, with a modal size $\sim 1-2 \mathrm{~km}$, and a steep drop off towards larger sizes. Most sporadic near-Sun comets are likely to follow the trends observed in the general comet population, if they have a cometary origin. Kreutz comet nucleus sizes, however, have been estimated to be meters to tens of meters in radius by a variety of modeling and observational techniques, e.g., MacQueen and St. Cyr (1991), Raymond et al. (1998), Uzzo et al. (2001), Iseli et al. (2002), Sekanina (2003), Bemporad et al. (2005), Knight et al. (2010). These sizes are similar to inferred from high resolution imaging with Hubble Space Telescope and/or thermal IR imaging with Spitzer Space Telescope, such as the 25-60 m range inferred for the fragments of split comets D/1999 S4 LINEAR (Weaver et al. 2001); the tens of m estimated for 73P/Schwassmann-Wachmann 3-C (Weaver et al. 2006; Reach et al. 2009), and the inferred 9-30 m range for 332P/lkeyaMurakami (Jewitt et al. 2016). Steckloff and Jacobson (2016) found similar results for their postulated parent chunks of striae via numerical modeling. This may suggest that individual Kreutz comets may represent the original discrete cometesimals out of which the Kreutz 


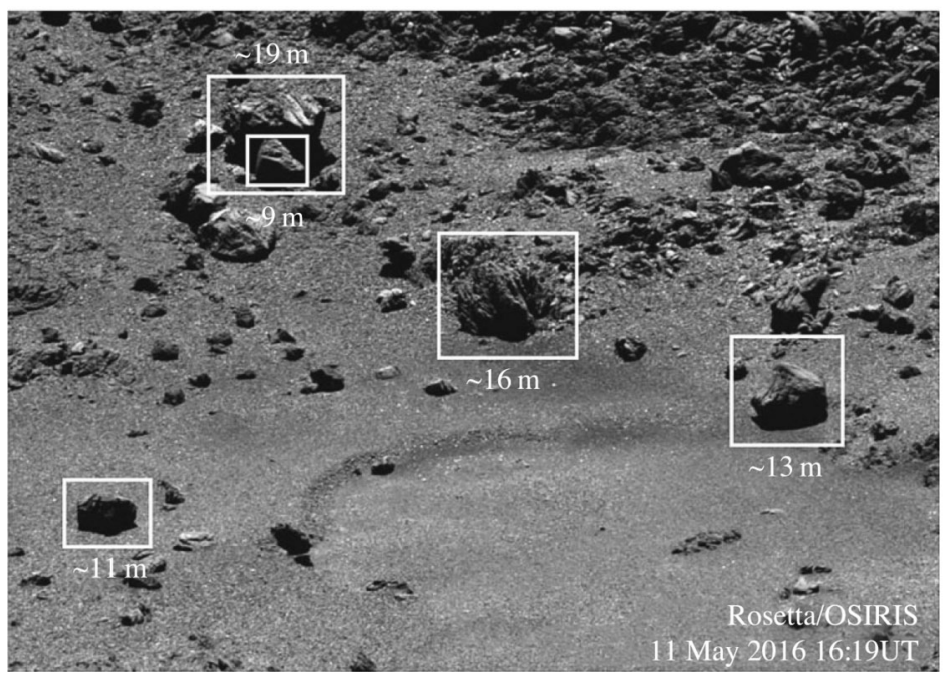

Fig. 14 Annotated Rosetta image highlighting large boulders on the surface of comet 67P with diameters similar to those estimated for SOHO comets (Battams and Knight 2016). Image credit: ESA/Rosetta/MPS for OSIRIS Team MPS/UPD/LAM/IAA/SSO/INTA/UPM/DASP/IDA

progenitor comet was formed. It is noted that the Rosetta mission has observed rounded nodules or spherules embedded in pit walls on 67P/Churyumov-Gerasimenko, each measuring 1-3 $\mathrm{m}$ across (Sierks et al. 2015). These spherules may represent the fundamental planetesimals that converged to form that nucleus, and could be analogous to the Kreutz fragments we now observe, though alternative interpretations of these spherules, which do not suggest that they reflect a primordial population, are also proposed (e.g., Auger et al. 2015). Loose "boulders" of similar scales have also been observed on 67P's surface (Fig. 14), and ejected into space (Fulle et al. 2016). No size estimates have been published for other groups of comets observed by SOHO-LASCO and STEREO-SECCHI, but they may be somewhat larger since some are seen during multiple apparitions without appearing noticeably fainter.

Knight et al. (2010) found the cumulative size distribution of Kreutz nuclei larger than $5 \mathrm{~m}$ in radius to be $N(>R) \sim R^{-2.2}$. Estimates of the cumulative size distribution of JFCs have suggested slopes of $\sim 1.9-2.7$, (e.g., Weissman and Lowry 2003; Meech et al. 2004; Tancredi et al. 2006; Snodgrass et al. 2011; Fernández et al. 2013) with most results agreeing at the lower end of this range. This slope is consistent with what would be expected from fragments produced in a collision, although the formation process is still debated. The size distribution of boulders on the surface of 67P, as measured by the Rosetta spacecraft, follow a slope of 2.7 (Pajola et al. 2015).

Lamy et al. (2013) derived a cumulative frequency distribution of the peak magnitude of the Marsden, Kracht, and Meyer groups plus "sporadic" comets. Each population has a steeper distribution than that of the Kreutz group members, i.e. a larger fraction of faint comets. This suggests that the Marsden, Kracht, and Meyer groups may have undergone more fragmentation than the Kreutz group, but this may be due to systematic differences such as comets' dwindling activity on repeat perihelion passages. No clear conclusions were drawn from the sporadic comets since they are far from constituting a uniform dataset.

A few comets have been seen in SOHO-LASCO or STEREO-SECCHI images on multiple apparitions; the two most compelling being "Kracht II" Comet 322P (Knight et al. 2016; 
see Sect. 3.1.4), and Marsden group Comet C/1999 J6 SOHO. Neither showed appreciable changes in lightcurve from one apparition to the next (Knight and Battams 2007; Lamy et al. 2013), suggesting that the nucleus size had not changed significantly from orbit to orbit. Both show asymmetries in brightness around perihelion that may be due to thermal lag or seasonal differences in nucleus illumination. Given the uncertainties in these observations inherent in and in the orbital solutions, such conclusions are highly speculative.

No information has been gleaned on the shapes of near-Sun comets' nuclei, nor would such information be readily informative. In passing, we do however note that the shape of C/2012 S1 ISON was partially constrained by Mars Reconnaissance Orbiter observations (Delamere et al. 2013).

\subsection{Rotation Rates}

A comet's spin period can have a significant influence on spatial variations in the degree of heating of its nucleus's surface. Comet sizes, shapes, and rotation periods (normally $\gtrsim 4$ hours), like rubble pile asteroids, seem to be controlled by tensile strength based on centrifugal force considerations. For example, 67P, which rotates every $\sim 12$ hours would split at $\lesssim 7$ hours due to its bilobate shape (Hirabayashi et al. 2016). Weissman et al. (2004) stated that the minimum period suggest bulk densities of $600 \mathrm{~kg} \mathrm{~m}^{-3}$ if nuclei were rubble piles held together only by self-gravity. Broad surveys of comet rotation periods agree with this (e.g. Lamy et al. 2004; Snodgrass et al. 2006; Kokotanekova et al. 2017). Steckloff and Jacobson (2016) found that a small $(100 \mathrm{~m})$ cometesimal held together by $10 \mathrm{~Pa}$ tensile strength would break apart at a rotation period of 52 minutes.

No rotation periods have been determined for near-Sun comets observed by SOHOLASCO and STEREO-SECCHI. This is most probably due to these instruments' large pixel scales damping small variations in brightness that might be due to varying activity or cross section as a nucleus rotates. The low resolution images do not permit the resolution of coma features which might be traced to reveal repetition, as is commonly used for comets near 1 AU (e.g., Farnham et al. 2007). Other observations, e.g., SOHO-UVCS or STEREO-SECCHI EUVI imaging spectroscopy, are of too short duration and of comets in an even harsher solar environment, and are therefore less likely to reveal rotation periods. Samarasinha and Mueller (2013) showed that near-Sun comets can have their rotation periods significantly altered and can be excited into non-principal axis rotation by the high outgassing rates near perihelion. Drahus (2014) argued that increasing coma optical depth could mitigate this to some extent, but nonetheless found that rotational disruption might explain the high frequency of destruction of long-period comets with $q<0.5$ AU noted by Bortle (1991). Rotational spin up could, therefore, be a significant cause of break up of near-Sun comet nuclei.

\subsection{Tensile Strengths}

Öpik (1966) provided the first strength estimate for sungrazers, based on observations of various Kreutz objects observed during 1843-1965. This value was $\sim 2 \mathrm{kPa}$, and was accompanied by discussion contemplating that "the cometary nuclei turn out to be weaker than all the listed materials except meteoritic dustballs... They could be loose structures, consisting of separate strong pieces but superficially welded together at the points of contact and readily detachable when some of the icy cement evaporates. In such a case it is quite possible that some pieces are of a much superior strength". Such ideas are still very valid today. 
More recently, specific strength estimates for sungrazers are the Uzzo et al. (2001) value of $10 \mathrm{~Pa}$ from the breakup of sungrazer C/2000 C6, and the Steckloff et al. (2015a) calculation of 0.5-9 Pa strength for C/2012 S1 ISON. These values are generally consistent with estimates for other comets, e.g. $<6.5 \mathrm{~Pa}$ for D/1993 F2 Shoemaker-Levy-9 (Asphaug and Benz 1996), < 1.8 Pa for 16P/Brooks 2 (Sekanina and Yeomans 1985), and the 10-200 Pa value for 67P/Churyumov-Gerasimenko (Hirabayashi et al. 2016).

These static tensile strength estimates should not be confused with the much higher estimates for dynamic strengths (e.g., Richardson et al. 2007) which are applicable in active processes, such as impact cratering. Philae instrument measurements from the surface of 67P are not comparable to the overall tensile strength estimates either because they were extremely localized measurements (several MPa; Spohn et al. 2015).

\subsection{Nucleus Composition}

Information about the composition of sungrazing comets is limited. Preston (1967) and Slaughter (1969) obtained spectra of C/1965 S1 Ikeya-Seki and identified emission lines of $\mathrm{O}, \mathrm{Na}, \mathrm{K}, \mathrm{Ca}, \mathrm{V}, \mathrm{Cr}, \mathrm{Mn}, \mathrm{Fe}, \mathrm{Co}, \mathrm{Ni}$, and $\mathrm{Cu}$, along with molecular lines of $\mathrm{CH}, \mathrm{CN}, \mathrm{C}_{2}$ and $\mathrm{C}_{3}$. The abundances of $\mathrm{Cr}, \mathrm{Mn}$, and $\mathrm{Ni}$ relative to $\mathrm{Fe}$ are broadly consistent with solar and meteoritic ratios, but the $\mathrm{Cu}$ abundance appears to have been anomalously high.

UV spectra of sungrazing comets from UVCS are dominated by H I Ly $\alpha$, but lines of $\mathrm{C}$ III, N V, O V, and Si III have been detected in the brightest sungrazers, giving a means to measure the composition (Ciaravella et al. 2010). Comet C/2003 K7 was observed at $3.4 \mathrm{R}_{\odot}$ (0.016 AU), so its grains were rapidly sublimated. The relative abundances of $\mathrm{H}$ : $\mathrm{C}$ : Si were approximately 1: 0.0035: 0.045. Spectra of C/2011 W3 Lovejoy show lines of O V, N V and Si III along with Ly $\alpha$ (Raymond et al. 2017, submitted).

Comet C/2011 W3 Lovejoy was also observed closer to the Sun with SDO-AIA (McCauley et al. 2013). When the comet was at $2.1 \mathrm{R}_{\odot}(0.010 \mathrm{AU})$, grains sublimated in a few seconds, so that the lines indicate the comet's total dust and ice composition. By comparing EUV bands dominated by oxygen lines with the $1600 \AA$ band dominated by C IV and the $171 \AA$ band that includes both $\mathrm{O}$ and Fe lines, they estimated that the $\mathrm{C}: \mathrm{O}$ ratio is about 0.07 and Fe:O is about 0.05. Pesnell and Bryans (2014) computed time-dependent ionisation models for the EUV emission of Comet Lovejoy, and they found that emission in the $1600 \AA$ A band was dominated by O II, O III, and O IV lines. However, a recalculation shows that $\mathrm{C}$ IV dominates the $1600 \AA$ band (P. Bryans, private communication). Thus the overall abundance ratios by number for $\mathrm{H}$ : $\mathrm{C}$ : N: O: Si: Fe are 1.0: 0.035: 0.004: 0.5: 0.015: 0.025 including both volatiles and dust.

\subsection{The Case of C/2012 S1 ISON}

We briefly discuss Comet C/2012 S1 ISON in particular, as thanks to its early discovery, it was studied more intensively than any other near-Sun comet. Multiple estimates of its nucleus size have been reported (e.g. Combi et al. 2014; Delamere et al. 2013; Lamy et al. 2014). Steckloff et al. (2015a) used the deconvolved daily average $\mathrm{H}_{2} \mathrm{O}$ production rates to indicate an effective radius of $0.58-0.85 \mathrm{~km}$, assuming an active fraction of unity. As most observations leading to these size estimates occurred beyond Mercury's orbit, and Comet ISON was entering the inner solar system for the first time, the size estimates do not inform our specific understanding of near-Sun comets. Tentative measurements of ISON's rotation period seem to indicate that it was $<24$ hours (Lamy et al. 2014; Knight and Schleicher 2015; Santos-Sanz et al. 2015). 
Fig. 15 Multiple exposures obtained on 2013 November 28 of C/2012 S1 ISON from $\mathrm{SOHO}$-LASCO-C3 and $\mathrm{C} 2$, with an SDO AIA image of the Sun. The comet approached from lower right. Post-perihelion, its dust tail because progressively fainter and more diffuse. Credits: ESA/NASA/SOHO/SDO/GSFC

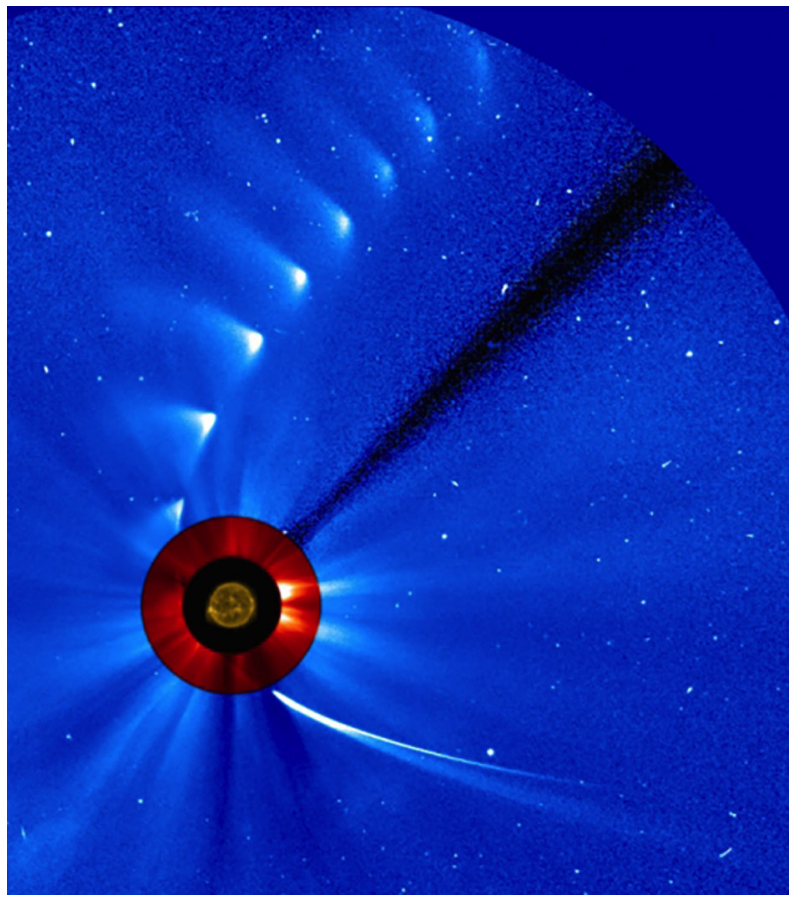

ISON is suspected to have broken up multiple times as it approached the Sun, evidenced by several changes in activity levels (Meech et al. 2013; Opitom et al. 2013). Gas production rates continued to increase until at least November 23 (0.33 AU, $71 \mathrm{R}_{\odot}$ from the Sun), implying significant mass loss and possible nucleus fragmentation during the week before perihelion.

Knight and Battams (2014) observed two dramatic and permanent brightening events of the nucleus with the STEREO-SECCHI HI1A instrument at heliocentric distances of 88 and $36 \mathrm{R}_{\odot}(0.41$ and $0.17 \mathrm{AU})$, which they interpret as additional fragmentation events. While Steckloff et al. (2015a) interpret this consistency between the size evolution of Comet ISON's nucleus and the three suspected fragmentation events to suggest that Comet ISON broke up in three distinct and separate events, Sekanina and Kracht (2014) believe that the nucleus underwent continuous erosion inward of $\sim 1$ AU. During the last two days before perihelion, the brightness slope of Comet ISON became even steeper, reaching a peak around November 28.1 (Knight and Battams 2014). No central condensation was observed post-perihelion, indicating that the comet did not survive its close encounter with the Sun (Fig. 15).

\section{Solar Insolation and Its Effects}

\subsection{Introduction}

The dominant physical processes at a comet are a strong function of heliocentric distance. Processes occurring at a nucleus near the Sun may well be in a totally different regime to those at $\sim 1 \mathrm{AU}$; when at temperatures $>273 \mathrm{~K}$, the sublimation physics we use at $1 \mathrm{AU}$ is not valid. Which cometary species are volatile is a function of heliocentric distance, as are 

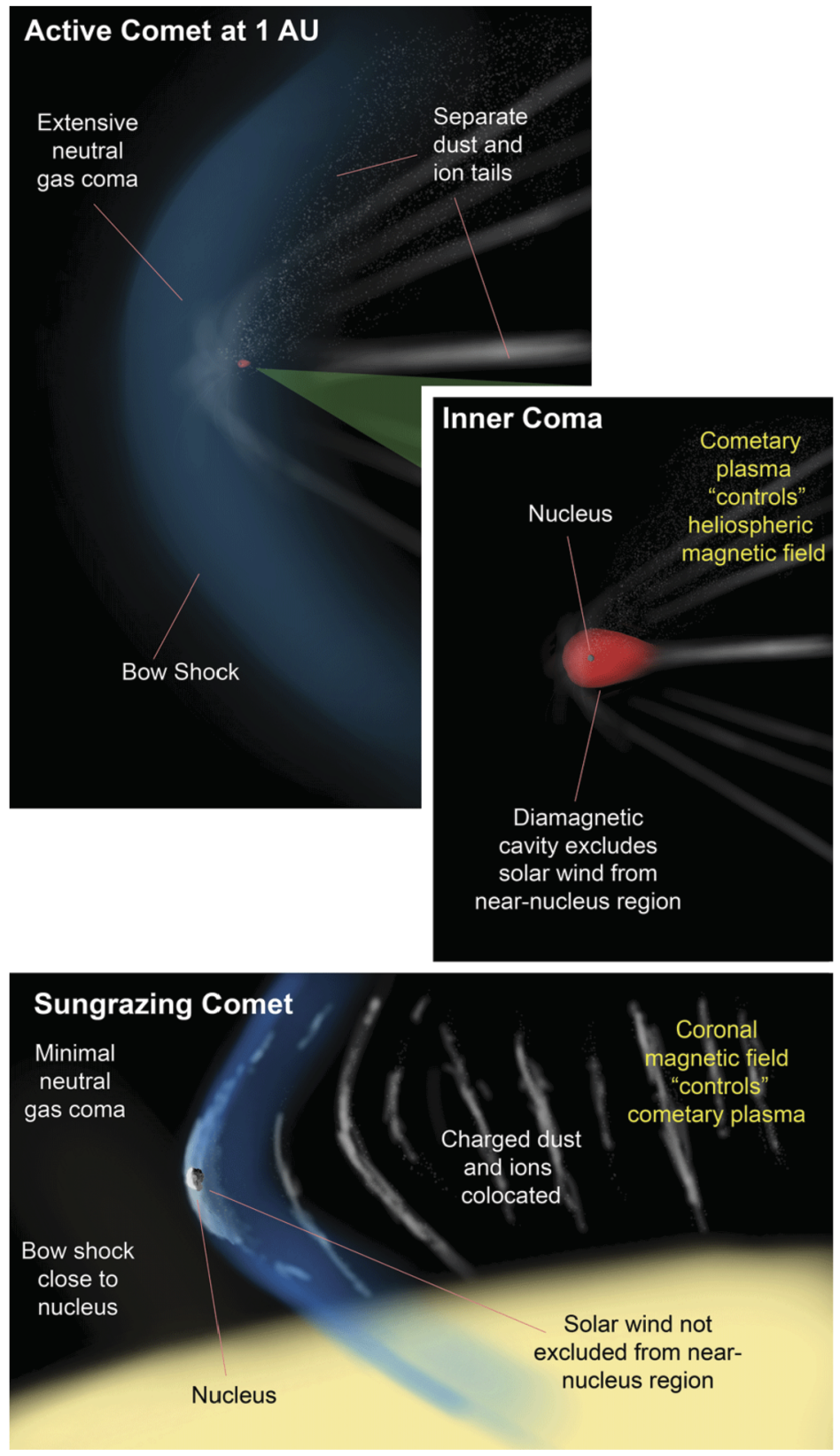

Fig. 16 Cartoons illustrating some of the major differences between a comet at $\sim 1$ AU from the Sun (top two panels), and a sungrazer. Note that features are not to scale

the timescales for dissociation and ionisation, which are both driven by photon fluxes as well as ion and electron impact. Overall, the comae and tails of the outermost near-Sun comets are likely to have processes largely scalable from 1 AU. This assumption is, however, likely to break down for sunskirters and sungrazers (Fig. 16). 


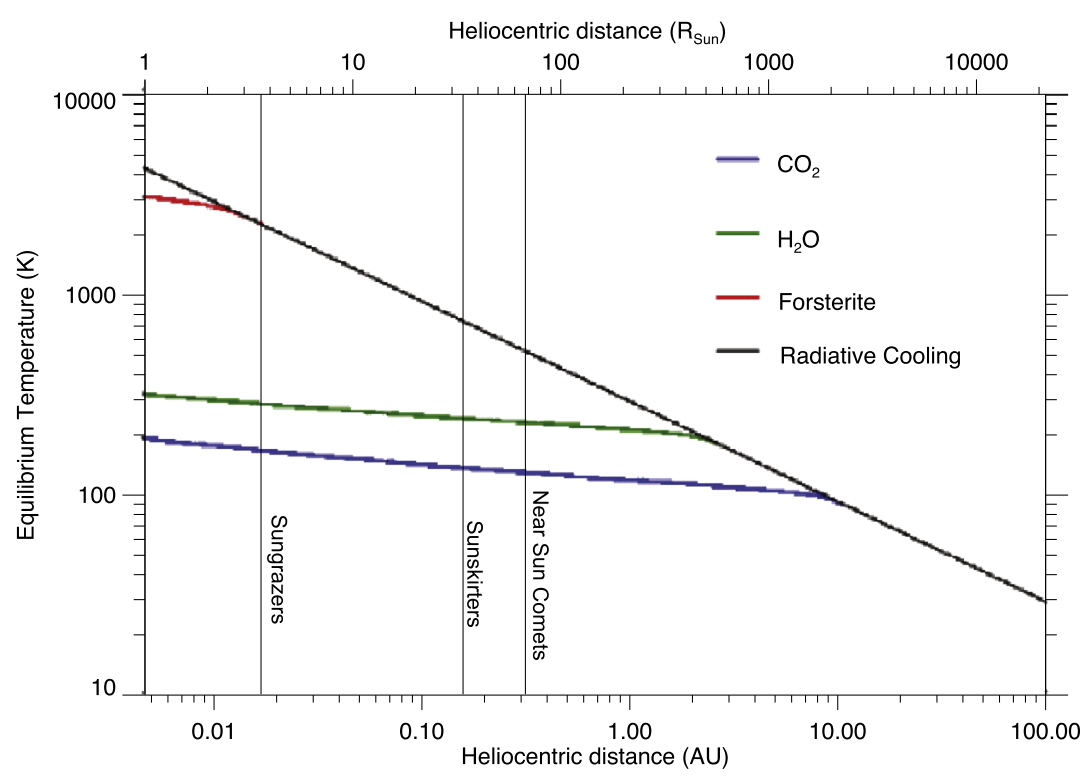

Fig. 17 Equilibrium temperatures of several materials against heliocentric distance, assuming the solar radiation flux follows an inverse square law point source assumption. The radiative cooling curve assumes that black body radiation is the dominant cooling mechanism of the cometary surface. Were the surface made of any of the materials represented here, that material would begin to sublimate if it became sufficiently heated. At this point, sublimative heat loss due to the latent heat of sublimation, would dominate the surface heat loss, keeping it cooler than thermal radiation alone. These transitions are represented by the points at which each material's temperature curve intersects the radiative cooling curve

When a comet comes within a few tenths of an AU from the Sun (a few dozen $\mathrm{R}_{\odot}$ ), the local temperatures can become so high that the refractory portion of the comet begins to sublimate in addition to volatile ices. Assuming adherence to the inverse square law, a cometary nucleus can reach sub-solar temperatures in Kelvin of

$$
T=400(1-A)^{1 / 4} / r_{\mathrm{H}}^{1 / 2}
$$

where $A$ is the albedo and $r_{\mathrm{H}}$ is the heliocentric distance (Fig. 17). Thus, a low-albedo object at $0.1 \mathrm{AU}$ will reach $1260 \mathrm{~K}$ whilst at $0.01 \mathrm{AU}$ it will reach $4000 \mathrm{~K}$.

Refractory organics begin decomposing and sublimating at $\sim 450 \mathrm{~K}$, metal sulfides at $\sim 700 \mathrm{~K}$, and silicates at $1000-1500 \mathrm{~K}$, depending on their $\mathrm{Mg} / \mathrm{Fe}$ content. An uptick in activity inbound at $\sim 0.7$ AU of dynamically new Comet C/2012 S1 ISON (Sect. 5.5) could suggest that the $\sim 450 \mathrm{~K}$ local temperature is enough to begin destroying some of the least refractory solids. These could include solid organic residues that may act as an adhesive. C/2011 W3 Lovejoy showed evidence for destruction of its dust tail as it came within $\sim 6.4 \mathrm{R}_{\odot}(0.03 \mathrm{AU})$ of the Sun's centre, but this tail regenerated after leaving this near-Sun region when newly released dust particles could again survive as solids. The tail of another large Kreutz sungrazer, C/1965 S1 Ikeya-Seki, was also noted to disappear between 8 and $4 \mathrm{R}_{\odot}(0.037$ and $0.019 \mathrm{AU})$, and was explained as being due to evaporation (see Huebner 1970 and references therein). One significant difference from comets near $1 \mathrm{AU}$ is the much higher density of the near-Sun plasma. Note that a comet's orbital speed of up to a few hundred $\mathrm{km} \mathrm{s}^{-1}$ is not a major influence in itself: the solar wind flows past comets far from the Sun at several hundred $\mathrm{km} \mathrm{s}^{-1}$. 
Several researchers have used comet thermal models to study the expected evolution of cometary nuclei on orbits with perihelia close to the Sun. Weissman (1983) estimated that a comet nucleus on a near-parabolic orbit would lose a surface layer up to $15 \mathrm{~m}$ thick for a sungrazing orbit. Results within an order of magnitude have been found using a variety of methods and assumptions by Iseli et al. (2002), Sekanina (2003), and Brown et al. (2011). However, the physics of those estimates likely breaks down at the near-Sun distances that we are discussing in this paper.

\subsection{Applicability of the Inverse Square Law}

There is one complication of studying objects very close to the Sun that we do not address in great detail in this work, but stress the need for it to be borne in mind in the context of sunskirting and especially sungrazing comets. This complicating factor is the breakdown in the inverse square law of solar radiation when close to a star.

The assumption that the Sun is a point source of radiation is a workable and reliable approximation in the vast majority of planetary science applications. Photons from the Sun can be considered to be arriving along parallel paths for most planetary bodies, and the solar constant can be reliably regarded as scaling proportionally to $r_{\mathrm{H}}^{-2}$. Close to the Sun however, these assumptions break down, e.g. Cassinelli et al. (1987), Brown et al. (1989), Huebner et al. (2007). The angular size of the Sun's disk becomes significant, meaning that photons can no longer be regarded as arriving at objects along directions parallel to each other. An object at low heliocentric distances will also be exposed to less solar radiation than expected under the inverse square law, as the visible disk of the Sun will be smaller than a solar hemisphere. Conversely, a greater proportion of a nucleus or dust grain's surface will be exposed to direct sunlight when the solar disk can no longer to be approximated as a point-like source. A recent treatment of this issue is included in the Appendix of Bryans and Pesnell (2016). The implications of the inverse square law breakdown include an adjustment to the effective magnitudes of heat flux and radiation pressure, and, in turn, the dynamics of any dust grains released from comets close to the Sun.

\subsection{Physical Processes Occurring at the Nucleus}

Efforts have been made to simulate gas and heat flow within the porous nucleus sub-surface layers (e.g., Huebner et al. 2006), but these processes are not fully understood. A key question concerning sungrazers is the degree of alteration and survivability of the nucleus, its erosion, and devolatilization. Does the thermal wave penetrate deep within the nucleus and devolatilize its material to significant depths, or is most of the thermal energy deposited at the surface but is then carried away by sublimating gas? Surface mass loss rates for sungrazers can be estimated by considering heating rates and latent heats of vaporization and sublimation of refractory materials thought to comprise the nucleus (e.g., olivine, pyroxene, forsterite, amorphous carbon, etc.). The penetration depth of the thermal wave determines the degree to which the pristine nature of the interior is preserved. Its penetration also determines whether the thermal stresses that develop in the sungrazer can disrupt the nucleus-an effect commonly observed in sungrazers - as well as the amount of erosion at the surface.

At perihelion, C/2011 W3 Lovejoy experienced temperatures of $\sim 2800 \mathrm{~K}$, sufficient to sublimate metals and refractories. Changes in surface composition and mineral phases (amorphous to crystalline, aqueous hydration, etc.), sintering, and the stability of the mantle (build-up versus blow-off due to increased gas production and higher velocity gas ejection) have yet to be investigated in detail. Other unknown material properties include interior 
changes in the stratification (composition, depths, etc.), pressure, temperature, porosity, and strength. Sekanina and Chodas (2012) propose that propagation of the thermal wave caused the disintegration of Comet C/2011 W3 Lovejoy a day or so after perihelion. When not near the Sun, larger particles in an active comet's dusty coma may fall back and mantle the nucleus. For comets close to the Sun however, this effect may be diminished significantly: dust lifetimes may be so short that any grains are destroyed before returning to the surface. Brown et al. (2011, 2015) have investigated the regimes where sublimation, ablation, and explosion dominate sungrazer destruction.

\subsection{Sublimation Processes}

At a cometary nucleus, incident solar energy is transferred into sublimative mass loss and cooling. Due to their low thermal inertias, little heat is conducted into the interior of comet nuclei. Equations describing the energy balance at the nucleus surface and their solutions have been given by many authors dating back to Watson et al. (1963) (see, e.g., Cowan and A'Hearn 1979; Weissman and Kieffer 1981). The latent heat of sublimation of water ice is very large, about the same as for rock (Mendis and Wickramasinghe 1975; Brown et al. 2011). While the equations generally used to compute vapour pressures may not be reliable at relatively high temperatures, volatile sublimation provides an effective cooling mechanism for keeping nucleus temperatures low, despite the extreme proximity to the Sun.

The equilibrium surface temperature for a point on a comet nucleus can be estimated by solving the 1D energy balance equation as shown in Weissman and Kieffer (1981). Steckloff et al. (2015b) simplified this numerical expression, resulting in the approximate energy balance equation

$$
F_{\odot}=\sigma T^{4}+\lambda(T) \sqrt{\frac{m_{m o l}}{2 \pi R T}} P_{(T)}
$$

where $F_{\odot}$ is the solar flux at the heliocentric location of the surface, $T$ the temperature, $\sigma$ the Stefan-Boltzmann constant, $\lambda(T)$ the temperature-dependent specific latent heat of sublimation, $m_{m o l}$ is the surface material's molar mass, $R$ the ideal gas constant, and $P(T)$ the material's temperature-dependent equilibrium vapour pressure. In Fig. 17, we plot the equilibrium surface temperatures as in Steckloff et al. (2015b), assuming negligible conduction into the cometary interior.

As the comet approaches the Sun, it warms up and is cooled predominantly through blackbody radiation. At some heliocentric distance, the material has warmed sufficiently for sublimation to begin, and for this to become the dominant cooling mechanism. The cometary volatiles $\mathrm{H}_{2} \mathrm{O}$ and $\mathrm{CO}_{2}$ are quite effective at limiting cometary temperatures to only a few hundred K even near the Sun's surface. However, were the nucleus to become devolatilized, then the refractory minerals within the nucleus (e.g., forsterite) may begin to sublimate as the nucleus temperatures rises above $\sim 1000 \mathrm{~K}$.

\subsection{Dust Sublimation and Dynamics}

Individual fine comet dust grains, entrained by sublimating ices, are lifted off the nucleus by the gas flow. They are then subjected to solar radiation pressure, and, apart from the population that is smaller than the wavelength of light, experience a radial force that accelerates them away from the Sun. Large particles and fragments from the nucleus generally follow the comet's orbit, but deviate from it due to radiation pressure. These deviating grains form dust tails, which are discussed further in Sect. 9.1. 
In the near-Sun environment, the trajectories of grains are further complicated by the more significant effect of drag due to collisions with coronal plasma particles. Depending on the environment, dust grains can plasma erode within seconds inside the corona. Within a few $\mathrm{R}_{\odot}$, all of the components of cometary material will at least start to vaporize. The lifetimes of individual grains are dependent on their masses and their trajectories.

Drahus (2014) argues that comae are generally all optically thick within $\sim 0.15 \mathrm{AU}$. The amount of solar energy absorbed or reflected by the coma is within half an order of magnitude of the amount of energy transmitted through the coma to reach the nucleus. The effects of a dust coma's optical thickness may be significant, and radiation pressure effects on dust grains within a thick coma can therefore potentially be minimal. Froehlich et al. (1987) and Notni and Thaenert (1988) proposed that optically-thick dust clouds could describe a bulk motion, following a collective trajectory equivalent to the effects of a radiation pressure force different to that expected for individual grains.

\subsection{Ionisation}

As soon as atoms or molecules leave the optically thick coma, they are exposed to ionising photons, hot electrons and ions. Far from the Sun, charge transfer reactions between solar wind protons and cometary hydrogen tend to dominate the ionisation rate, with photoionisation close behind. At the higher coronal densities and temperatures being considered here, ionisation by electron collisions is faster than photoionisation. The rate coefficients for ionisation of neutrals are $\sim 10^{-7} \mathrm{~cm}^{3} \mathrm{~s}^{-1}$. In the corona above $2 \mathrm{R}_{\odot}(0.0093 \mathrm{AU})$, the densities decline from about $10^{6} \mathrm{~cm}^{-3}$, so that ionisation times are tens to thousands of seconds. Near perihelion for Kreutz comets, coronal densities are $\sim 10^{8} \mathrm{~cm}^{-3}$, so ionisation times are below a second. The ionisation rate coefficients for higher ionisation states are much smaller, so that the $\mathrm{O}^{4+}(\mathrm{O} \mathrm{V})$ and $\mathrm{O}^{5+}(\mathrm{O} \mathrm{VI})$ ions seen in SDO-AIA images (Bryans and Pesnell 2012; McCauley et al. 2013) persist for tens or hundreds of seconds.

\section{Influences on a Near-Sun Cometary Nucleus}

\subsection{Tidal Forces}

Comets passing close to the Sun, in particular sungrazers, are subject to strong tidal forces. The Kreutz group is suspected to result from the tidal disruption of a larger progenitor comet on one or more previous close perihelion passages. Unequivocal tidal disruptions are rare. The only known relatively recent examples are those of D/1993 F2 Shoemaker-Levy 9 in 1991 (Chodas and Yeomans 1996) and 16P/Brooks 2 in 1886 (Sekanina and Yeomans 1985); these were both disrupted due to close approaches to Jupiter.

Tidal disruptions yield unique information about the internal structures of comets, potentially revealing if they are weakly bound "fractal aggregates" (Donn and Hughes 1986) or "primordial rubble piles" (Weissman 1986), and if they are collisional fragments of larger bodies (e.g., Schlichting et al. 2013; Morbidelli and Rickman 2015).

Weissman et al. (2012) modeled a sungrazing comet following the orbit of Kreutz Comet C/1965 S1 Ikeya-Seki assuming a rubble-pile nucleus of radius $1 \mathrm{~km}$, using the dynamical model previously used by Movshovitz et al. (2012). Their results were qualitatively similar to the observed size distribution of the Kreutz group, and suggested that multiple returns are needed by the parent comet and its initial fragments to provide the observed temporal separation of major fragments. They found that non-rotating sungrazing comets passing 
within $2 \mathrm{R}_{\odot}$ of the Sun (0.014 AU from the centre) and with bulk nucleus densities ranging from 100 to $1,000 \mathrm{~kg} \mathrm{~m}^{-3}$, would disrupt and form a string of cometesimals that would gravitationally recombine to form one or more nuclei. This was particularly true for the low density, $<600 \mathrm{~kg} \mathrm{~m}^{-3}$ nuclei passing within $\sim 1.6 \mathrm{R}_{\odot}$ of the photosphere (heliocentric distance of $0.012 \mathrm{R}_{\odot}$ ). At larger perihelion distances and higher bulk densities the string of cometesimals was not very long and they all combined back into a single nucleus. Such cometesimals would be subject to rapid evaporation.

Analysis of the resulting planetesimals' size distribution showed that there were only a few large sub-nuclei and a large number of very small nuclei, with the largest number being single cometesimals. This is qualitatively similar to the observed Kreutz group where there are fairly few large, naked-eye objects and a large number of objects with radii of $\lesssim 10 \mathrm{~m}$. Analysis of the temporal distribution of the returning orbits showed that they were spread over $\sim 70$ years, i.e. less than the time that the Kreutz group has been systematically observed. This suggests that multiple returns are needed by the parent comet and its initial fragments to provide the time span for observed Kreutz group comets, which is currently $\sim 135$ years.

The passage of Kreutz Comet C/2011 W3 Lovejoy challenges the simple rubble pile model, as it may have survived for hours to a few days past perihelion (Sekanina and Chodas 2012). Gundlach et al. (2012) suggest that if this was the case, it survived because the optically thick coma caused a sublimative confining pressure which effectively increased the nucleus's strength.

\subsection{Nucleus Disruption}

Many potential processes for nucleus disruption have to be considered. The oft-cited sizeindependent Roche limit distance inside which the tidal separation force from the Sun exceeds the binding self gravity force is only relevant to separation of parts of a body held together solely by self-gravity, such as a rubble or sand pile. Even in that case the classical Roche limit result is for circular orbits and needs modification for transient parabolic encounters, and also for the disruption-enhancing effects of nucleus rotation. In the case of objects of finite tensile or shear strength, tidal disruption can only occur for objects large enough for the tidal force to exceed the strength. For example, for a tensile strength of $10-100 \mathrm{~Pa}$, objects have to be $0.3-1 \mathrm{~km}$ in size before self gravity is more important than strength. Further discussion of these issues are to be found in Sect. 11.3, and by Davidsson (1999, 2001), Bear and Soker (2013, 2015), Brown et al. (2017).

\subsection{Other Potential Influences}

Another force sometimes invoked as enhancing nucleus disruption (e.g., Sekanina and Kracht 2015; Steckloff et al. 2015a) is ram pressure arising from unstable internal sublimative outflow. Gundlach et al. (2012) have, however, argued that in the sungrazing environment, the cometary coma may become optically thick, resulting in nearly uniform global sublimation from the surface, which creates a confining sublimative reaction pressure that may inhibit disruption. One factor which is definitely not relevant to nucleus survival, though often erroneously emphasised, is the corona's $2 \times 10^{6} \mathrm{~K}-2 \mathrm{MK}-$ kinetic temperature. Although very hot, the coronal plasma is so tenuous that its heat flux is less than the energy flux of sunlight at Earth.

A recent topic of discussion (Sekanina and Kracht 2015; Steckloff et al. 2015a,b) is the influence of non-gravitational forces on sungrazer nuclei nearing perihelion. The standard 
non-gravitational force model was derived by Marsden et al. (1973) to account for the apparent divergence of comets from their purely gravitationally defined orbits. Described by some as a "rocket effect", non-gravitational perturbations result from asymmetric momentum transfer due to preferential volatile sublimation on a nucleus's Sun-facing hemisphere. Weissman (1979) showed that nongravitational forces can substantially change comets' orbital semi-major axes, particularly for those with small $q$.

Sungrazers and sunskirters undergo intense thermal stresses when their surfaces are heated to $>1,000 \mathrm{~K}$ or more near perihelion. At these temperatures, all volatile ices and hydrocarbons will sublimate, along with some metals and silicates. Indeed, even at the greater heliocentric distance of comet 67P/Churyumov-Gerasimenko, thermal stresses are suspected of fracturing and weakening surface materials, leading to cliff collapses and mass wasting events (e.g., Grün et al. 2016; Vincent et al. 2016). Thus, while thermal stresses will not disrupt and disperse a comet nucleus, they appear to be able to weaken and break nuclei into smaller pieces.

Comets have been known to split at random times for no obvious reason (e.g., Weissman 1980; Boehnhardt 2004). These events are not well explained but one leading explanation is rotational break-up due to spin-up from asymmetric outgassing on the nucleus, which may have been responsible for C/2012 S1 ISON's apparent breakup prior to perihelion (e.g., Samarasinha and Mueller 2013). Sekanina (2000, 2002a) used the motions of pairs of temporally clustered Kreutz comets to argue that non-tidal fragmentation occurs at very large heliocentric distances (tens of AU) in addition to tidal fragmentation near perihelion. Although no mechanisms have been conclusively shown to split comets at such distances, Sekanina suggested that extensive cracking occurred near the previous perihelion which sufficiently weakened the nuclei such that rotational tension eventually caused breakups.

\section{Comae}

\subsection{Introduction}

The proximity of near-Sun comets to the Sun results in very different physical processes and observing conditions than for comets studied near 1 AU. At near-Sun distances, photoionisation rates are substantially faster, sublimation is not restricted to normally volatile ices, and different chemical reactions may dominate. Additionally, the large pixel scales of $S O H O$-LASCO and STEREO-SECCHI, limited or non-existent spectroscopic capabilities, wide spectral bandpasses, and the bright sky background/foreground due to sunlight scattered by the plasma and dust components of the solar corona make it difficult or impossible to study the coma in ways analogous to typical cometary studies. In this section we discuss what occurs in near-Sun comets' comae and how they are studied.

\subsection{Neutral Comae}

The comparison of the magnitudes of sungrazing comets in the orange and clear $\mathrm{SOHO}$ LASCO filters indicates very strong contributions of emission lines of sodium (e.g., Knight et al. 2010). As discussed in Sect. 4.3, comparison of the brightness in multiple bandpasses using the combined capabilities of SOHO and STEREO provides basic compositional information.

Neutral sodium emission may also be seen in cometary spectra, and is particularly prevalent in the spectra of near-Sun comets. Observing cometary sodium in spectra may be extremely challenging as, unless the cometary sodium emission is sufficiently Doppler shifted 
from the telluric sodium lines, a cometary signal may not be distinguishable. Some spectra show two distinct neutral sodium features at different velocities (Cremonese 1999; Leblanc et al. 2008), possibly suggesting multiple sources of sodium.

The origin of neutral sodium in comets is undetermined. An ion source is speculated to produce sodium at much higher velocities than those observed, and is not generally supported. A consensus on a dust and/or nuclear ice source of neutral sodium in different comets has not yet been reached (reviews include Cremonese 1999 and Cremonese et al. 2002), though Rosetta did detect sodium in dust grains (Schulz et al. 2015). Observations of neutral cometary material, particularly sodium, may also allow details to be inferred about the chemical and physical processes occurring in cometary comae. Ellinger et al. (2015) suggested, from the results of chemical simulations, that an ice source for neutral sodium could suggest aqueous alteration in the comet nucleus.

At most visible wavelengths, cometary comae in the corona are dark as they are cold compared to the coronal material, and block the Sun's emission. The neutral cloud around Comet C/2011 N3 was seen to absorb in the EUV bands of SDO-AIA (Schrijver et al. 2012).

\subsection{Dust Comae}

Traditional models of dusty gas flow in a cometary atmosphere (e.g., Konno et al. 1993; Boice et al. 2002) include gas-drag force (momentum transfer), heat and mass exchange between gas and dust, radiative heating and cooling for dust particles, particle size distribution (dozens of discrete grain sizes), and fragmentation of grains (each grain size with a different lifetime). These models illuminate relevant physical processes for gas-dust interactions at distances outside the near-Sun environment, such as distributed gas sources from the dust, mass-loading of gas by the dust, and energy exchange between gas and dust components. Since no such model exists for near-Sun comets, the traditional models must be adapted by adding heats of vaporization of the refractory dust grains and thermal fragmentation mechanisms for the intense thermal environment of the near-Sun environment. Recent work by Boice (2017) attempts to address these enhancements with the SUISEI model. Model profiles of the number densities, velocities, and temperatures of dust as a function of size, and fragmentation rates could then be related to existing observations.

An underutilized aspect of the SOHO-LASCO and STEREO-SECCHI data is the ability to make polarization measurements that can reveal information about, as well as changes in, the coma's dust properties. The polarization signal depends on phase angle and wavelength (e.g., Kolokolova et al. 2004), so the high phase angles at which many near-Sun comets are observed are unique. Polarization observations are acquired approximately daily with the orange filter for $\mathrm{SOHO}$-LASCO's $\mathrm{C} 2$ and $\mathrm{C} 3$, but are acquired significantly more frequently with STEREO-SECCHI's COR1 and COR2 (the exact imaging cadence has varied during the mission). Almost no comets are observed in COR1 due to its limited field of view, but tens of comets have been observed in COR2 so it is likely that there are now sufficient data for a systematic study of polarization data of near-Sun comets. To date, published works utilizing these data have been at non-sungrazing distances, e.g., 96P/Machholz 1 at $\sim 0.15 \mathrm{AU}$ $\left(32 \mathrm{R}_{\odot}\right.$ ) (Grynko et al. 2004) and P/2003 T12 SOHO at $\sim 0.60 \mathrm{AU}\left(129 \mathrm{R}_{\odot}\right)$ (Hui 2013).

\subsection{Coma Chemistry}

Physico-chemical models of cometary comae have traditionally been developed for comets near 1 AU and beyond (see e.g., Rodgers et al. 2004; Boice and Wegmann 2007). Only one early chemical model by Swift and Mitchell (1981) was adapted for near-Sun comets. This 
calculated the coma chemistry of a sunskirting comet at 0.125 AU with single-fluid physics, including opacity effects for the photolytic reactions. Since then, there have been no detailed studies of coma chemistry at small heliocentric distances. Studies of comets in the inner heliosphere include MHD and hybrid modeling (e.g., Jia et al. 2011) but without detailed chemistry and linkage to the nucleus interior. Various physical and chemical processes need to be considered when modeling near-Sun comets' comae. In combined models, the physical and chemical structures of the multi-fluid flow are calculated self-consistently (Boice and Wegmann 2007) as a function of heliocentric distance.

The major differences between the dust, gas, and plasma environment at an active comet beyond about $0.5 \mathrm{AU}\left(107 \mathrm{R}_{\odot}\right)$ and that at a sungrazer include:

- Much more intensive solar UV radiation at a sungrazer, so photochemical and photoionisation reactions are more important

- Enlargement of the collisionally dominated inner coma at a sungrazer, hence the increased importance of gas-phase chemistry in this region

- Increased optical depth effects in a sungrazer's coma with higher gas production and densities

- Coupling with the solar wind much stronger for comets near the Sun, which directly affects plasma boundaries and structures

- The near-Sun thermal environment results in the volatilization of refractory comet dust. This leads to a distributed source of gas and plasma throughout the sungrazer's coma

- Sputtering by energetic ions and the dense plasma environment near the Sun must be taken into account

- Insolation plays a more important role in the energy balance at a sungrazer's surface, leading to increased sublimation (devolatilization), ablation, and possibly explosion.

At heliocentric distances of $\sim 1 \mathrm{AU}\left(215 \mathrm{R}_{\odot}\right)$, gas is cooled by inelastic collisions between electrons and water molecules. As water photodissociation becomes complete for sungrazers, fine-structure cooling eventually dominates. As the degree of ionisation of the coma increases from $<1 \%$ (a neutral coma) to near 100\% (a plasma coma), cation-atom scattering will also become important.

An understanding of the plasma coma of sungrazers likely needs a 3-D multi-fluid MHD model, such as CASIM3D (Benna and Mahaffy 2007), with the addition of detailed chemistry. At the high temperatures experienced by sungrazers, dust sublimation physics needs to also be considered to add another distributed source of coma gas.

A multi-fluid gas dynamics model with chemistry (see, e.g., Rodgers et al. 2004; Boice and Wegmann 2007) can solve the fluid dynamic equations for the mass, momentum, and energy of separate neutral fluids (such as atomic and molecular $\mathrm{H}$ and the heavier bulk fluid), ions, and electrons. In the inner coma, the gas expands, cools, accelerates, and undergoes many photolytic and gas-phase chemical reactions requiring tracking hundreds of daughter species in the simulation. These codes handle the transition to free molecular flow and describe the spatial distribution of neutral species in the outer coma. They provide estimates of the cometocentric abundances of the coma gas species; velocities of the bulk gas, light atomic and molecular hydrogen, with escape, and electrons; gas and electron temperatures; column densities to aid comparison with observations; coma energy budget; attenuation of the solar irradiance; and other observable quantities.

The solar radiation field initiates most processes that occur in cometary comae. UV photons photodissociate and ionise the original parent molecules, producing second-generation reactive radicals, ions, and electrons. These ions and radicals can then react with other species to form third-generation species. Photoelectrons are an additional source of ionisation (and dissociation) via impact reactions (Ip 1985; Boice et al. 1986). Due to the enhanced 
insolation levels and solar wind densities experienced by near-Sun comets, these photolytic and chemical processes have increased importance, leading to unusually high coma gas temperatures and pressures, gas expansion velocities, and optical depth effects, all of which need to be accounted for self-consistently. Simulations of a comet following its orbit from $2.5 \mathrm{AU}$ to $0.3 \mathrm{AU}$ pre-perihelion show dramatic increases in the gas velocity and temperature within about $0.5 \mathrm{AU}$, with dramatic effects on the chemistry, optical depth, and other coma properties (Boice 2017). As the sungrazer approaches the Sun-for example, within the $1.191 \mathrm{R}_{\odot}$ $(0.0055 \mathrm{AU})$ perihelion distance of C/2011 W3 Lovejoy-the coma shields the nucleus via optical depth effects, reduces sputtering on the surface. It also acts as insulation during the rotation cycle, and may lead to recondensation on the night side. These processes and others (e.g. sputtering of the cometary dust grains and the surface by the solar wind plasma, evaporation of dust grain minerals, suprathermal photochemistry due to the extreme UV irradiation, etc.) need to be incorporated into current comet coma models. The basic physics and incident solar radiation geometry of sungrazing comets for studying processes such as photodissociation and photoionisation near the Sun has been given by Huebner et al. (2007).

Swift and Mitchell (1981) found that the UV optical depth (Ly $\alpha \tau=1)$ increases significantly as a comet approaches the Sun. At $1 \mathrm{AU}\left(215 \mathrm{R}_{\odot}\right)$ this region corresponds to the inner $45 \mathrm{~km}$ surrounding the nucleus while at $0.125 \mathrm{AU}\left(26.9 \mathrm{R}_{\odot}\right)$, the region has enlarged to $4400 \mathrm{~km}$. This results in photodissociation products forming further from the nucleus at small heliocentric distance $\left(r_{\mathrm{H}}\right)$ and then falling off steeply. Photo lifetimes of cometary species are proportional to $r_{\mathrm{H}}^{-2}$ so, for example, water photodissociation timescales vary from $8.6 \times 10^{4} \mathrm{~s}(1 \mathrm{AU})$ to $8.6 \times 10^{2} \mathrm{~s}(0.1 \mathrm{AU})$. These effects alter chemical networks. Neutral-neutral reactions become increasingly important with decreasing heliocentric distance, whereas they are usually ignored in typical comets at $1 \mathrm{AU}$ and beyond. They further found that the major $\mathrm{OH}$ source in near-Sun comets is $\mathrm{CH}_{3} \mathrm{OH}$ in the inner coma, and $\mathrm{H}_{2} \mathrm{O}$ is the outer coma parent. Since photodissociation of $\mathrm{HCN}$ and $\mathrm{CH}_{3} \mathrm{CN}$ strongly depend on $\mathrm{Ly} \alpha$ radiation, they become outer coma sources of $\mathrm{CN}$, while $\mathrm{HC}_{3} \mathrm{~N}$ is the dominant inner coma parent. The chemistry of $\mathrm{C}_{2}$ and $\mathrm{C}_{3}$ are similarly affected at small $r_{\mathrm{H}}$, whereas formation of $\mathrm{NH}_{2}$ is not affected much by the UV optical depth.

\subsection{Comet-Solar Wind Interactions}

In the strongly ionising environment of the intense near-Sun radiation field and dense inner heliospheric and coronal plasma, interactions between cometary material and the solar wind are highly influential compared to their effects on comets at $1 \mathrm{AU}$. Fresh ions formed at a comet are entrained in the solar wind via the process of ion pickup and are carried antisunward, forming an ion or plasma tail. The addition of mass to the solar wind leads to its deceleration, draping the frozen-in heliospheric magnetic field (HMF) as it passes through the comet's coma (Alfvén 1957). This magnetic field topology is believed to be completely unaffected by the cometary nucleus itself; the Rosetta and Philae spacecraft measured no intrinsic magnetic field at Comet 67P/Churyumov-Gerasimenko (Auster et al. 2015). The ions can be observed remotely, generally through resonance fluorescence processes. Active comets possess a diamagnetic cavity - a region where the outward flow of cometary ions is strong enough to exclude the solar wind (e.g. Goetz et al. 2016). A contact surface separates the contaminated solar wind from the diamagnetic cavity.

A model that consistently describes the detailed physics and chemistry for the inner coma and the solar wind interaction with the outer coma (see, e.g., Boice and Wegmann 2007) does not yet exist for sungrazing comets. Such a model would be important for investigating the conditions of the corona, solar wind, and inner heliosphere. There are broadly two types of 
Fig. 18 Colour-coded density of a sungrazer in the low solar corona. The white lines trace the distorted coronal magnetic field (Jia et al. 2014)

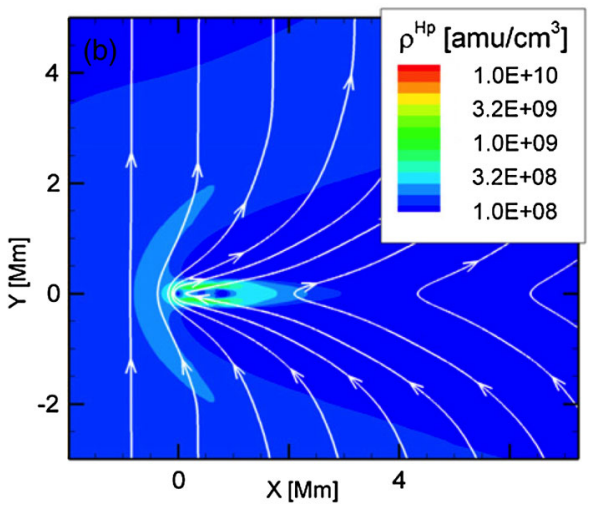

codes available: 2-D hydrodynamic codes with very detailed chemical kinetics (e.g., Wegmann et al. 1998, 2004; Wegmann and Dennerl 2005) and 3-D MHD codes with simplified chemistry (e.g., Wegmann 1995, 2000). These can predict coma properties, such as the gas, ion, and electron densities, velocities, chemical species abundances, electron temperatures (using multiple electron populations), gas temperature, magnetic field configuration, and the dynamics of neutrals and ions inside and outside the diamagnetic cavity contact surface, consistent with properties of the inner coma and the onset of the plasma tail. Other properties of the coma and the mass-loaded solar wind, such as electron density and temperature, are calculated using detailed excess photon and collision energies and electron cooling.

Jia et al. (2014) have made a significant step forward in simulating a sungrazer in the low corona at a heliocentric distance of $\sim 0.006 \mathrm{AU}\left(1.29 \mathrm{R}_{\odot}\right)$ using a static multifluid MHD model. The modelled comet had a water production rate of $2.6 \times 10^{30} \mathrm{molec} \mathrm{s}^{-1}$. The chemistry of water group species was included. An upstream pileup region with a bow shock $\sim 1 \times 10^{6} \mathrm{~m}$ upstream was observed, caused by the ionisation process. The peak number density was found to be $\sim 10$ times the local coronal density.

When a diamagnetic cavity is present, direct sputtering of nucleus material no longer takes place. Jia and collaborators' model predicts that such a cavity does surround the nucleus under the modelled conditions, as well as a small $\mathrm{O}^{+}$cloud of number density around 100 times that of the local corona. High $\mathrm{O}$ charge states were found to dominate in the tail, and the magnetic field was seen to drape in response to the mass loading (Fig. 18). Cometary ions carried downstream form the ion tail, which is discussed in more detail in Sect. 9.3.

\section{Tails}

\subsection{Dust Tails}

\subsubsection{Overview}

Comet tails provide valuable information about the characteristics of the dust grains; they also contain evidence of nucleus rotation, dust particle fragmentation, and possibly of solar wind interactions. These grains are almost certainly electrically charged, and smaller ones will be most strongly influenced by the solar wind. Most sungrazers do not develop dust tails that can be resolved with current instrumentation, and of those that do, observations haven't yet revealed the influence of the Lorentz force on the dust (Sekanina 2000). That influence 
Table 5 Sublimation distances for a range of minerals, plus water ice for comparison. Adapted from Mann et al. (2004) and references therein

\begin{tabular}{lll}
\hline Material & Sphere & Fluffy \\
\hline Quartz & $1.5-4 \mathrm{R}_{\odot}$ & - \\
FeO-poor obsidian & $1.9-7 \mathrm{R}_{\odot}$ & $2.5-3 \mathrm{R}_{\odot}$ \\
FeO-rich obsidian & $2.9-6 \mathrm{R}_{\odot}$ & - \\
Glassy carbon & $4 \mathrm{R} \odot$ & $4 \mathrm{R}_{\odot}$ \\
Graphite & $\leq 5 \mathrm{R} \odot$ & $\leq 2 \mathrm{R} \odot$ \\
Crystalline Mg-rich pyroxene & $5 \mathrm{R} \odot$ & $5 \mathrm{R}_{\odot}$ \\
Amorphous Mg-rich pyroxene & $5.5-6.5 \mathrm{R} \odot$ & $5-6.5 \mathrm{R} \odot$ \\
Basalt & $6 \mathrm{R} \odot$ & - \\
Andesite & $9-10.5 \mathrm{R} \odot$ & $2.5-3 \mathrm{R} \odot$ \\
Crystalline Mg-rich olivine & $10 \mathrm{R} \odot$ & $9.5-11 \mathrm{R} \odot$ \\
Amorphous Mg-rich olivine & $13.5-15.5 \mathrm{R} \odot$ & $12-15 \mathrm{R} \odot$ \\
Astronomical silicate & $14 \mathrm{R}_{\odot}$ & - \\
Iron & $11-24.3 \mathrm{R} \odot$ & - \\
Magnetite & $10-40 \mathrm{R} \odot$ & - \\
Water ice & $<2.8 \mathrm{AU} ;<602 \mathrm{R}_{\odot}$ & - \\
\hline
\end{tabular}

must however be significant, as the Lorentz force has to be invoked to explain some dust tails observed far from the Sun (Kramer et al. 2014).

Bright ordinary comets, such as C/2006 P1 McNaught, display extensive striated dust tails thought to be evidence of dust fragmentation. To interpret such observations, a comprehensive dusty coma model is needed that contains Finson-Probstein dust entrainment, fragmentation with multiple particle sizes, and development of the dust tail via synchrones and syndynes (see Sect. 9.1.2), e.g., Sekanina 2000.

Cometary dust eventually becomes part of the interplanetary dust environment, contributing to the solar F-corona and other dust populations (Mann et al. 2004; Nesvorný et al. 2010). However, much of the dust observed close to the Sun originates at larger distances and spirals inwards due to the Poynting-Robertson effect. Grains that remain in the extreme near-Sun environment are eroded by sputtering by the dense solar wind plasma and may eventually sublimate. Dust sublimation depends on the release distance and the grains' composition (Table 5), with olivines sublimating near $12 \mathrm{R}_{\odot}(0.056 \mathrm{AU})$, while pyroxenes sublimate near $6 \mathrm{R}_{\odot}(0.028 \mathrm{AU})$ (Kimura et al. 2002).

Chochol et al. (1983) reported the possible detection of visible wavelength emission lines from sublimating dust grains following the perihelion passage of C/1979 Q1 (SOLWIND). The ionised products of the dust will be carried outwards from the Sun by the solar wind, and may provide much of the inner source of pickup ions observed further from the Sun (Bzowski and Królikowska 2005; Mann 2010). The ions that are released from sublimating dust in the corona quickly reach higher charge states, the charge state depending on the release distance from Sun (Mann and Czechowski 2005). For example, doubly ionised carbon and silicon have been detected with UVCS (Povich et al. 2003; Ciaravella et al. 2010).

\subsubsection{Dust Dynamics and Resultant Tail Structures}

Dust within a comet experiences a gravitational attraction to the Sun and a radiation pressure that acts anti-sunward. Because the gravitational force scales as the grain volume while the 
latter scales as its surface area, the net acceleration is size and shape dependent. Since both solar gravity and radiation pressure scale as the inverse square of $r_{\mathrm{H}}$, the ratio of a grain's radiation to gravitational accelerations can be parameterized by the dimensionless quantity $\beta=a_{\text {rad }} / a_{\text {grav }}$. Small dust grains are relatively susceptible to radiation pressure and have higher values for $\beta$ than do larger grains. If $\beta>1$, then radiation pressure overcomes solar gravity, and the grain is accelerated anti-sunward. If a swarm of dust grains with various $\beta$ parameters was suddenly produced near the Sun, it would extend anti-sunward to form a linear dust feature organized by $\beta$ parameter, with larger grains (generally lower $\beta$ ) remaining closer to the Sun, and smaller (higher $\beta$ ) grains further away.

The effects of radiation pressure are clearly present at all heliocentric distances. However, it appears that near-Sun conditions may be particularly conducive to the production of dust populations that form structures accentuated by radiation pressure effects, including dramatic variations in dust production through nuclear activity outbursts and grain fragmentation. The greater orbital velocities of near-Sun comets compared to objects further away also leads to the larger physical spread of each dust tail, which can accentuate substructures within them. Finally, near-Sun comets' proximity to the Sun also makes usually subtle dust tail features more obvious, due to sunlight's strong forward-scattering phase dependence (Sect. 4.4). This increases near-Sun comet tails' brightnesses when on the same side of the Sun as the observer.

Large-scale linear dust structures within cometary dust tails tend to align with either the coma or are offset from this direction, broadly towards the Sun. Structures that align with the coma are likely formed from the diurnal release of $\sim 1-100 \mu \mathrm{m}$ dust from the nucleus (Kharchuk and Korsun 2010), which is transported to the tail through radiation pressure. Because they result from dust that is released almost synchronously, these structures are called synchrones, or synchronic bands, and are common in high dust production rate comets.

The linear structures that do not align with the comet's head, called striae, or sometimes psuedosynchronic bands, are poorly understood. Figure 19 demonstrates the difference between synchronic bands and striae. The latter have only been observed in relatively few, predominantly high production rate-comets (see Table 2 for examples). While observed in comets with perihelia out to $\sim 1 \mathrm{AU}$, such as C/1995 O1 (Hale-Bopp), striae are most commonly seen in comets nearer to the Sun. Sekanina and Farrell (1980) proposed three steps in stria formation:

1. a parcel of material is released from the nucleus and drifts away due to radiation pressure

2. the parcel disperses or fragments

3. the resulting fragments stream away from one another according to their $\beta$ parameters.

Although Nishioka (1998) proposed that step (2) could be a continuous fragmentation cascade, rather than a discrete fragmentation (or dispersion) event, the general model of Sekanina and Farrell (1980) agrees well with observations of several comets (Sekanina and Farrell 1980; Pittichová et al. 1997; Sekenina and Pittichová 1997). The primary difference in later models of stria formation (Sekanina and Farrell 1980; Hill and Mendis 1980, 1981; Froehlich and Notni 1988; Kharchuk and Korsun 2010; Jones and Battams 2014; Steckloff and Jacobson 2016) is in the details of the first two steps. Sekanina and Farrell (1980) proposed that parent grains have highly elongated shapes, and spin up as a result of uneven radiation pressure. However, the mass of each stria would require unreasonably long grains. Hill and Mendis $(1980,1981)$ proposed that the electrostatic charging of parent grains would chip away at the irregular tips and bumps of these particles.

Froehlich and Notni (1988) suggested that the parcels of cometary material take the form of $\sim 1000 \mathrm{~km}$-wide, optically thick clouds of grains with a narrow range of $\beta$. Each cloud 


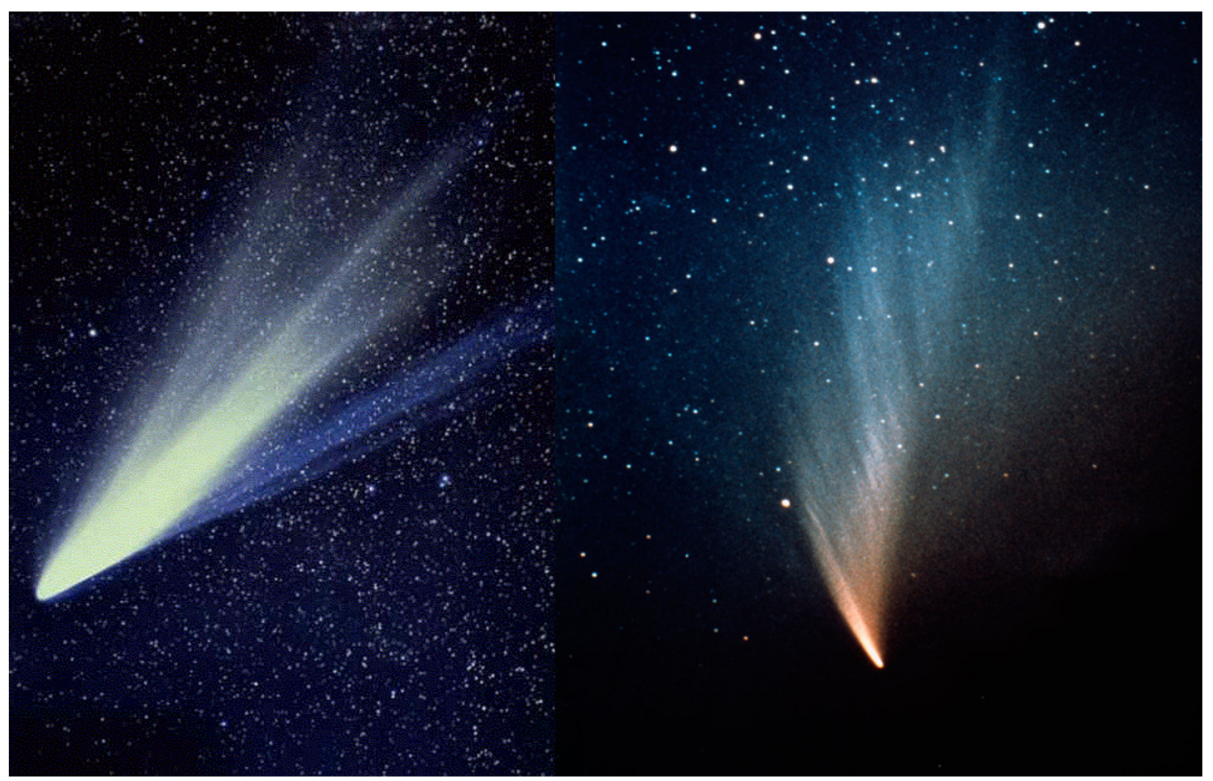

Fig. 19 Comet C/1975 V1 (West) on two different dates, when its dust tail was dominated by synchronic bands (left; image by Observatoire de Haute-Provence), and striae (right; image by P. Stättmayer/ESO). Synchronic bands, which point towards the nucleus position, can be explained by variations in a nucleus's dust production rate, whereas the prevailing theories for striae involve the break-up of dust well after it has left the nucleus. Note that striae are not aligned with the nucleus position

would travel as a unit under the effects of radiation pressure, with the allowed range of $\beta$-parameters being determined by the cloud's optical thickness. Grains in the sunward region of the swarm, especially those of higher $\beta$, would be accelerated antisunward, into the cloud. When within the higher optical depth region, the radiation pressure acting on them decreases. Meanwhile, other grains take their place at the sunward part of the cloud, where they also are forced antisunward. Grains therefore continually cycle through positions in the swarm. The grains' transverse velocities are thermodynamically most likely to have a Maxwell-Boltzmann distribution. Without a mechanism for confining the dust in two free dimensions, this mechanism is unlikely to maintain a coherent swarm for long enough to form a stria, unless the grains are unusually dynamically cold. Such $\sim 1000 \mathrm{~km}$ wide optically thick clouds have also yet to be observed.

Kharchuk and Korsun (2010) attempted to model the C/2006 P1 striae through dust production rate variations alone. However, although their Monte Carlo simulation results had strong qualitative similarities to the observed linear features, their orientations were very different, and matched those of synchrones.

Steckloff and Jacobson (2016) proposed a stria formation mechanism that avoids the issues with swarms by assuming that a stria is formed from a single parent object $\sim 10 \mathrm{~m}$ in radius. Instead of driving parent grains away from the nucleus with radiation pressure, their mechanism relies on the reaction pressure from sublimating volatiles (Steckloff et al. 2015 b). This can be 4-5 orders of magnitude stronger than radiation pressure in the inner Solar System. Additionally, it relies on a rotational fragmentation cascade that is driven by sublimative torques (Steckloff and Jacobson 2016). While this newly-proposed mechanism 
Fig. 20 Comet McNaught (C/2006 P1) imaged using the SOHO-LASCO C3 orange filter, and displaying a distinct neutral sodium tail. Celestial north is downwards in this view

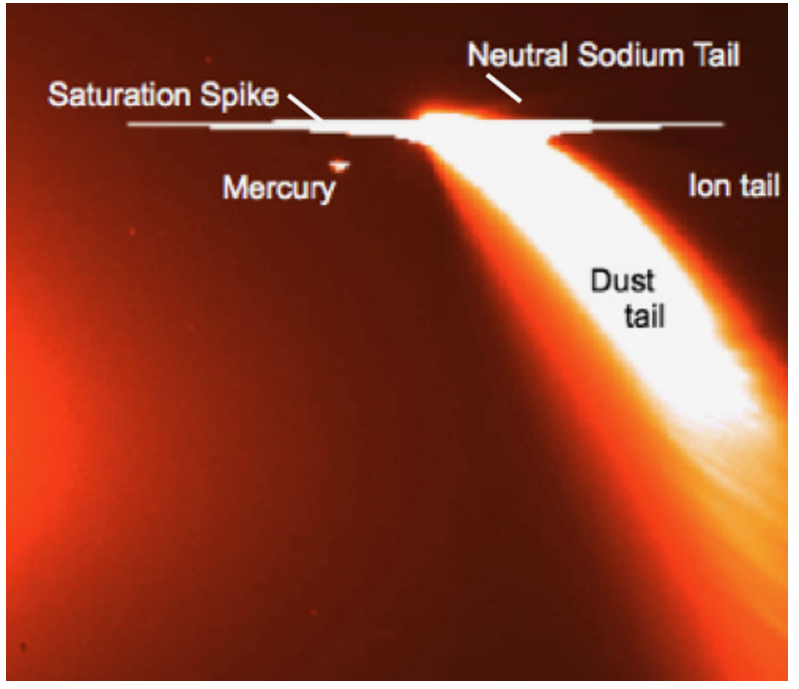

is able to recreate the striae of C/1975 V1 West (as described in Sekanina and Farrell 1980), it is unknown whether it can meet all observational constraints.

Lastly, Pittichová et al. (1997) found that the periodicity of parent grain ejection at C/1996 O1 Hale-Bopp is consistent with the nucleus's rotation period. This suggests that the mechanism driving parent grain ejection is thermally driven and responds to the diurnal heating of nucleus active areas. However, neither the mechanics of parent grain ejection nor the size of the ejected grains is understood.

\subsection{Neutral Tails}

Near-Sun comets often display sodium tails. Such a tail was first clearly imaged in the past few decades at C/1995 O1 Hale-Bopp in 1997, following an earlier detection at C/1957 P1 Mrkos with an objective prism (Cremonese et al. 1997 and references therein). C/1995 O1 was at $0.98 \mathrm{AU}\left(211 \mathrm{R}_{\odot}\right)$ when its tail was discovered, clearly not in the near-Sun regime. Subsequent sodium tail discoveries have all been in near-Sun comets (e.g. Fig. 20), suggesting that very high production rates, such as those of C/1995 O1, or higher temperatures are required to liberate sufficient atoms from cometary material for a distinct tail to form.

Like dust, the morphology of neutral sodium tails is determined by the balance between radiation pressure and gravitational forces. Sodium is the most likely neutral species to be observed as a tail primarily due to the high efficiency (high $g$ factor) of the sodium D1 and D2 electronic transitions (at $5896 \AA$ and $5900 \AA$, respectively). However, sodium is also present in the Sun's atmosphere, where it absorbs radiation, producing the Fraunhofer lines. The heliocentric velocity of an individual sodium atom therefore determines the radiation pressure it experiences. The lifetimes of sodium atoms due to photoionisation are very short, approximately $1.7 \times 10^{5} \mathrm{~s}$ at $1 \mathrm{AU}$ (Cremonese et al. 1997). Simulating sodium tails' formation and morphology is therefore a complex task. Computational modelling involving orbital motion and observational perspectives are required, e.g. Birkett (2017), in order to successfully interpret the observations.

Neutral sodium tails have also been observed at near-Sun comets through use of the $\mathrm{SOHO}$-LASCO coronagraphs, $\mathrm{C} 2$ and $\mathrm{C} 3$. Each instrument is capable of taking images us- 
ing orange and blue filters (bandpasses shown in Table 3 and Fig. 9), and a comparison of these images sometimes allows a distinct neutral sodium tail to be discerned (Fig. 20).

It has been speculated that other neutral tails may be observed, provided the abundance of the species is relatively high, its lifetime against ionisation is relatively long and its electronic transitions are sufficiently efficient. See Table 1 in Cremonese et al. (2002) for a list of key neutrals potentially observable as comet tails. Fulle et al. (2007) interpret the neutral tail observed at Comet C/2006 P1 McNaught using STEREO-SECCHI-HI1-A and -B as comprising neutral iron. The inferred $\beta$ parameter agrees with that of iron. In addition, the HI1 bandpass does not allow for sodium emission to be detected, but there does exist a window at neutral iron emission line wavelengths (Fig. 9).

\subsection{Ion Tails}

The magnetic field draping pattern imposed on the heliospheric magnetic field (HMF) at a comet's head is carried downstream to form an induced magnetotail: a structure qualitatively similar to the tails of planetary magnetospheres such as that of Earth, in that it is characterized by two magnetic lobes in which the magnetic field is oriented in generally opposite directions, e.g., Szegö et al. (2000). The magnetic lobes are separated by a neutral or current sheet, where the direction of the magnetic field reverses, e.g. Jockers (1985).

Most magnetized planets' magnetotails are largely fixed in configuration, as their topology is primarily controlled by the parent planets' magnetic fields. Induced magnetotails, however, are almost completely controlled by the orientation of the HMF upstream of the pickup ion source: the cross-tail current sheet lies in the plane perpendicular to the HMF orientation upstream. Cometary current sheet orientations are, as a result, highly variable, reflecting changes in the upstream HMF. The current sheet orientation undergoes smooth rotations as well as abrupt switches as a result of varying HMF conditions convecting through the tail. Cometary ions are concentrated in this sheet, and its orientation with respect to a remote observer can change a tail's appearance dramatically, appearing thin and sharp when viewed edge-on, and broad and diffuse when face-on. This picture was largely predicted prior to being confirmed by the in situ observation of cometary ion tails.

The HMF draping pattern at near-Sun comets may differ significantly from that typically observed at 1 AU. Near Earth orbit, the HMF, following the Parker Archimedian spiral, is typically oriented at $\sim 45^{\circ}$ to the radial direction. Comets that venture closer to the Sun experience a less tightly-wound $\mathrm{HMF}$, which tends to be more radial. In extreme cases, the component of the magnetic field perpendicular to the radial direction may be small enough for a draping pattern to no longer form. This would result in an ion tail lacking the induced magnetotail morphology, and thus a cross-tail current sheet. Such tails would appear more diffuse than those observed at active comets further from the Sun. This effect may disappear around perihelion for all comets, including sungrazers, as the comet's velocity is then primarily perpendicular to the radial direction.

Sungrazers often display dust tails, but distinct ion tails have only been observed in a small number of objects, such as C/2011 W3 Lovejoy. It should be noted that this may be largely explained by the fact that sungrazers' high orbital velocities during perihelion passage lead to the superposition of the ion and dust tails. SOHO-LASCO and STEREOSECCHI have successfully imaged the ion tails of several non-sungrazing near-Sun comets, including those of C/1996 B2 Hyakutake, and C/2002 V1 NEAT.

The Ulysses spacecraft serendipitously encountered several comets' ion tails during its 1990-2009 mission, including those of near-Sun Comets C/1996 B2 Hyakutake (Jones et al. 
2000; Gloeckler et al. 2000) and C/2006 P1 McNaught (Neugebauer et al. 2007). Ion composition measurements differed from those made at comets further from the Sun were consistent with a near-Sun source. This included a high proportion of atomic as opposed to molecular ions, and a greater proportion of multiply-ionized species (Gloeckler et al. 2000; Neugebauer et al. 2007). A particularly complex magnetotail structure was detected downstream of McNaught over several days, the interpretation of which is complicated by the scale of the structure: different convected solar wind features were observed inbound and outbound. At both tails, weak but significant increases and decreases in HMF magnitude were observed before and after the tail crossing, respectively. These may be bow shock or wave crossings; in the latter case, they were separated by 18 days, suggesting that Ulysses encountered a linear sample of solar wind affected by the comet's presence more than $7 \mathrm{AU}$ in length. Shou et al. (2015) modelled the interaction of C/2006 P1 with the solar wind over a range of heliocentric distances, finding good agreement overall with Ulysses tail composition measurements. The response of ion tails' orientations to changing solar wind parameters is covered in Sect. 10.5.

\section{Comets as Probes of the Corona and Solar Wind}

\subsection{Overview}

A comet passing through the corona provides a means to estimate the plasma parameters at each point along its path, which is a useful complement to remote sensing imaging or spectroscopic observations which provide the average properties along lines of sight through the optically thin corona. If the outgassing rate is small, so that a given mass of material lost from the comet interacts with a much larger mass of coronal gas, the cometary atoms can be viewed as test particles dropped into the corona.

The test particle approximation is valid for most of the comets observed by UVCS, which are small and have modest outgassing rates. In that case, neutral hydrogen atoms from the comet undergo charge transfer with coronal protons, producing a population of neutral particles with approximately the velocity distribution of the coronal protons. The width of the Ly $\alpha$ profile is a measure of the coronal proton temperature, and the rate of spatial spreading of Ly $\alpha$ emission is a second means to measure the coronal temperature. The Doppler shift of the Ly $\alpha$ centroid is the line-of-sight component of the coronal outflow speed. The Ly $\alpha$ emission decays as the neutrals are ionised by electron collisions, so the decay time scale is a measure of the electron number density. Once the density, temperature and flow speed have been determined, the Ly $\alpha$ intensity can be converted into the outgassing rate of the comet. By equating the outgassing rate times the energy required to sublimate $\mathrm{H}_{2} \mathrm{O}$ to the absorbed solar radiation, one obtains an estimate of the size of the nucleus.

In its almost continuous period of observation from 1996 to 2013, UVCS observed 12 sungrazing comets (Raymond et al. 1998; Uzzo et al. 2001; Bemporad et al. 2005, 2007; Ciaravella et al. 2010; Giordano et al. 2015). All these comets belong to the Kreutz group. In most cases, orbits computed from LASCO observations were used to position the UVCS slit so that the comet moved across the slit at several heights between 2 and $10 \mathrm{R}_{\odot}$. Since the speeds of the comets are known, it is possible to reconstruct a raster-like image from each series of exposures. In addition, from spectral data it is possible to determine the temperature and the velocity of the cometary gas along the line of sight. An example of a reconstructed comet image is shown in Fig. 21. 

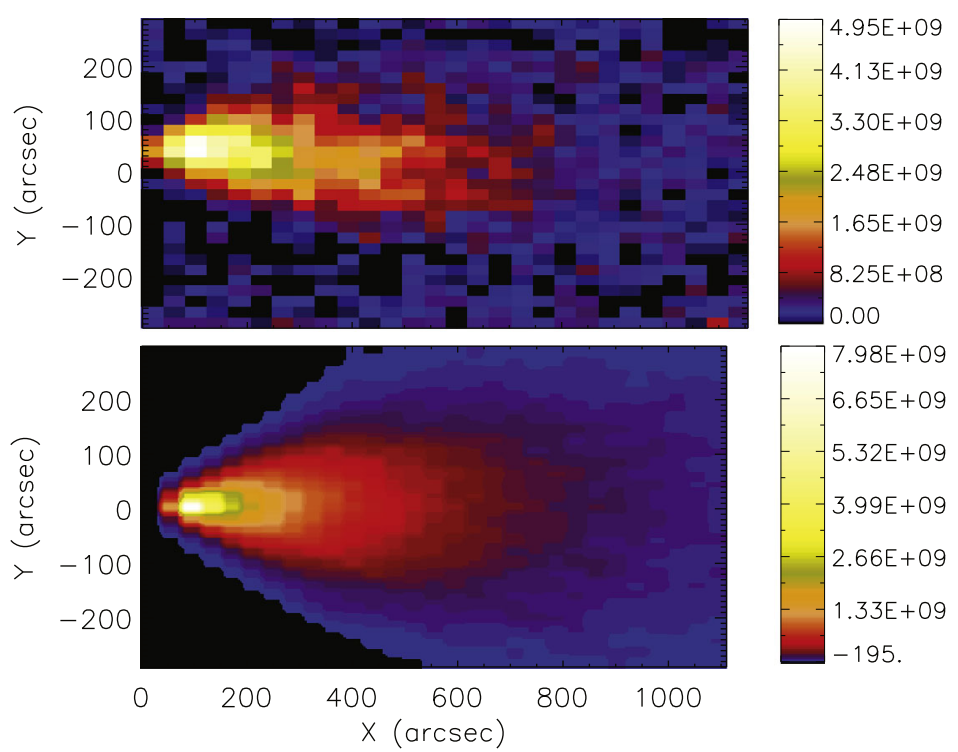

Fig. 21 Top: Image of C/2002 S2 in Ly $\alpha$ reconstructed from a series of UVCS spectra at $5.99 \mathrm{R}_{\odot}$. The color bar shows the intensity in units of photons $\mathrm{cm}^{-2} \mathrm{~s}^{-1} \mathrm{sr}^{-1}$ (Giordano et al. 2015). Bottom: A Monte Carlo simulation of the Ly $\alpha$ image

\subsection{Interpretation of $\operatorname{Ly} \alpha$ Observations}

The Ly $\alpha$ line is formed by scattering of chromospheric Ly $\alpha$ photons from hydrogen atoms produced by the photodissociation of water. The $\mathrm{H}$ atoms leave the coma with speeds of up to $24 \mathrm{~km} \mathrm{~s}^{-1}$ resulting from the photodissociation process. Thus these "first generation neutrals" move at the comet speed plus a random speed up to $24 \mathrm{~km} \mathrm{~s}^{-1}$. Since the comet is approaching the Sun at a speed larger than $200 \mathrm{~km} \mathrm{~s}^{-1}$, the absorption profile is Doppler shifted away from the chromospheric emission line, and the scattering is severely Doppler dimmed (Swings effect). The hydrogen atoms can be ionised by collisions with coronal electrons, photo-ionised by the UV solar radiation or can undergo charge transfer with coronal protons, resulting in a population of neutrals moving at the thermal and bulk speeds of the corona. These "second generation" neutrals are generally less severely Doppler dimmed, so they dominate the observed emission in the regions of slow solar wind.

A summary of the outgassing rates and nucleus radii obtained so far for the five published sungrazing comets is given in Table 6, along with the derived coronal densities. However, in addition to the perturbation of the corona by the comet mentioned above, there are other complications that can affect the estimates. Some comets appear to fragment, as indicated by a rapid increase in outgassing rate (Uzzo et al. 2001) or a split tail (Bemporad et al. 2005), and a very slowly decaying component of the Ly $\alpha$ emission is attributed to neutralization of protons by particles in the dust tail (Bemporad et al. 2005).

Of the comets observed before C/2011 W3 Lovejoy, five have been studied in detail. Four have outgassing rates of 10 to $800 \mathrm{~kg} \mathrm{~s}^{-1}$. Assuming that all but a few $\%$ of the solar radiation incident on the nucleus goes into sublimating water, that translates into effective nucleus radii of $\sim 3-20 \mathrm{~m}$. The largest comet observed by SOHO-UVCS before C/2011 W3 Lovejoy was C/2003 K7, with an estimated nucleus radius of 30-60 m (Ciaravella et al. 2010). 
Table 6 Outgassing Rates, Nucleus Radii $\left(\mathrm{R}_{n}\right)$ and coronal densities derived from UVCS observed sungrazing comets. References: (1) Raymond et al. (1998), (2) Uzzo et al. (2001), (3) Bemporad et al. (2005), (4) Giordano et al. (2015), (5) Ciaravella et al. (2010)

\begin{tabular}{lllllll}
\hline Date YYYY-MM-DD & Comet & $r_{\mathrm{H}}\left(\mathrm{R}_{\odot}\right)$ & $\mathrm{Ndot}\left(\mathrm{s}^{-1}\right)$ & $\mathrm{R}_{n}(\mathrm{~m})$ & $N_{e}\left(\mathrm{~cm}^{-3}\right)$ & Reference \\
\hline 1996-12-23 & C/1996 Y1 & 6.80 & 0.13 & 3.4 & & $(1)$ \\
2000-02-10 & C/2000 C6 & 3.88 & 0.71 & 3.0 & $1.0 \times 10^{5}$ & $(2)$ \\
& & 4.68 & 1.35 & 5.7 & $6.8 \times 10^{4}$ & \\
& & 5.88 & 0.33 & 3.4 & $6.5 \times 10^{4}$ & \\
2001-02-(6-7) & & 6.47 & 0.13 & 2.5 & $2.6 \times 10^{3}$ & \\
& $\mathrm{C} / 2001 \mathrm{C} 2$ & 4.98 & 0.59 & 7.8 & $3.0 \times 10^{4}$ & $(3)$ \\
& & 4.98 & 0.29 & 5.4 & $1.6 \times 10^{4}$ & \\
2002-09-18 & 3.60 & 8.20 & 20.3 & $7.0 \times 10^{4}$ & \\
& & & & \\
$2003-05-24$ & $\mathrm{C} / 2002 \mathrm{~S} 2$ & 5.99 & 1.12 & 9.0 & $1.23 \times 10^{4}$ & $(4)$ \\
\hline
\end{tabular}

Fig. 22 Apparent $\mathrm{V}$ magnitude of $\mathrm{C} / 2002 \mathrm{~S} 2$ determined from $\mathrm{SOHO}$-LASCO $\mathrm{C} 2$ and $\mathrm{C} 3$. The black dots represent the apparent magnitudes derived from UVCS observations of H I Ly $\alpha$ intensity arbitrarily scaled to the LASCO apparent magnitude range

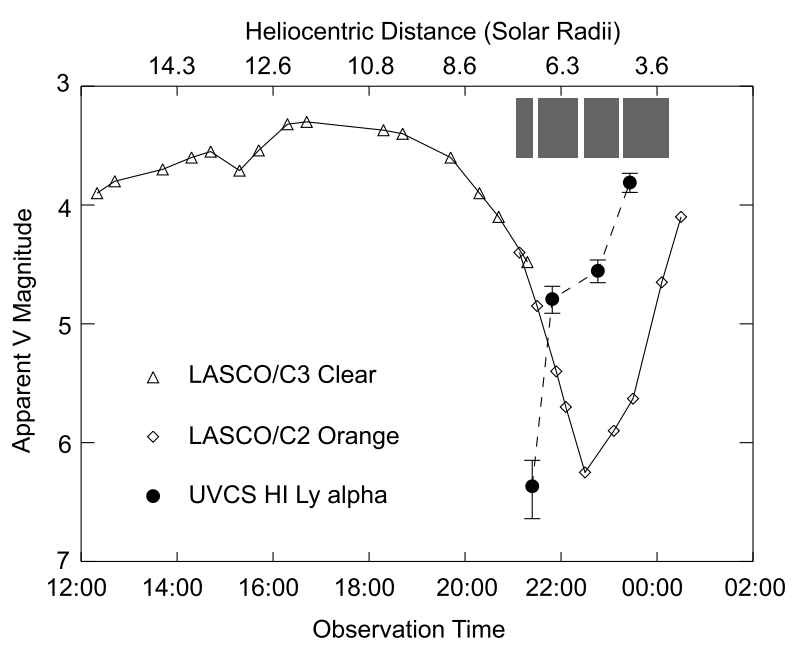

The most complete analysis of UVCS observations of a sungrazing comet so far is that of C/2002 S2 (Giordano et al. 2015). This comet was observed at 4 heights between 6 and $8.5 \mathrm{R}_{\odot}$. Figure 21 shows the image of the comet in Ly $\alpha$ reconstructed from a series of $120 \mathrm{~s}$ exposures at the lowest observed height. This comet showed two remarkable properties. First, it brightened steadily in Ly $\alpha$ inbound, while its optical brightness measured by LASCO dropped by 2 magnitudes and then recovered (Fig. 22). This presumably resulted from changes in grain vaporization or the destruction of molecules. Second, the Ly $\alpha$ line showed a blue shift to the North of the comet trajectory and a red shift to the South (Fig. 23). The blue shift is the line-of-sight component of the solar wind velocity, while the red shift is the line-of-sight component of the comet speed. The spatial separation probably results from the motion of protons formed by charge transfer and collisional ionisation along the magnetic field, but a detailed model is needed to confirm this. Simple models of the formation of pickup hydrogen, followed by isotropization of the velocity component 
Fig. 23 Doppler speed images of the H I Ly $\alpha$ comet emission from UVCS observations at $5.99 \mathrm{R}_{\odot}$ for $\mathrm{C} / 2002 \mathrm{~S} 2$. The color bar shows the Doppler speed in $\mathrm{km} \mathrm{s}^{-1}$

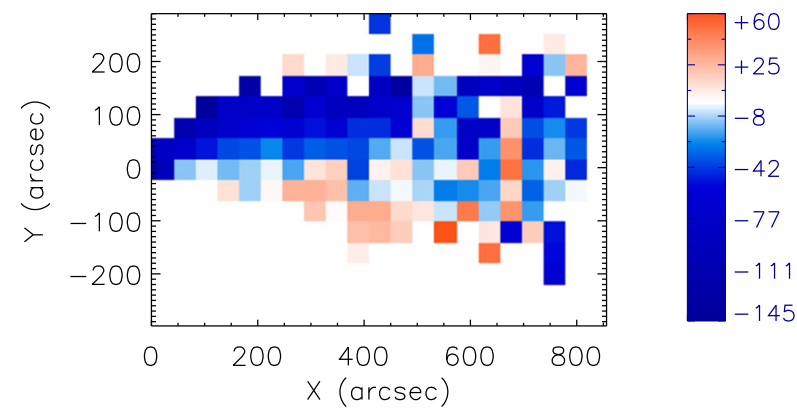

perpendicular to the field and subsequent charge transfer to form "third generation" neutrals can match the overall character of the intensity and Doppler shift images.

To better understand the relationships between the observed H I Ly $\alpha$ spectral emission and the coronal plasma's physical parameters, Giordano et al. (2015) constructed a threedimensional time-dependent Monte Carlo simulation based on following $\mathrm{H}$ atoms from photodissociated water as they are ionised or undergo charge transfer and scatter chromospheric Ly $\alpha$ photons. From this, they inferred the outgassing rates and coronal parameters, such as outflow wind velocity, electron density, and proton temperature. The bottom panel of Fig. 21 shows the Monte Carlo simulated H I Ly $\alpha$ intensity image that best reproduces the observation at $5.99 \mathrm{R}_{\odot}$. The simulation matches the observation reasonably well, though it tends to give too sharp an intensity peak when the comet crosses the slit.

Such models provide means to interpret $\operatorname{Ly} \alpha$ comet observations in terms of the solar wind speed and density in the region between the solar surface and the closest approach of the upcoming Solar Orbiter and Parker Solar Probe missions. The METIS coronagraph on Solar Orbiter is expected to produce Ly $\alpha$ images that will be useful for this purpose (Bemporad et al. 2015); see Sect. 13.3.1 for additional details.

\subsection{Interpretation of Narrow Band EUV Images}

C/2011 W3 Lovejoy acted as a probe of the solar corona at even lower heights, revealing the magnetic field and density structure at each point along its path. It was seen in most of the bands of the $S D O$-AIA instrument, and the images are remarkable. They show a series of striations at angles to the comet trajectory, with the intensity peak of each striation moving away from the comet's path and spreading over the course of a few minutes. Figure 26 shows a composite of 6 individual images taken after perihelion, with off-limb emission enhanced using a radial filter.

As suggested by Bryans and Pesnell (2012), emission in most of the SDO-AIA bands is dominated by the oxygen ions O III through O VI, with some contribution of Fe in the $171 \AA$ and $131 \AA$ bands, and C IV dominating the $1600 \AA$ band. In the bands dominated by the lower ionisation stages, such as the $304 \AA$ and $211 \AA$ bands, the striations are very short, reflecting the rapid ionisation through the O III and O IV ionisation states.

The striations (Fig. 24) clearly indicate motion of the ions along the local magnetic field (Downs et al. 2013). The directions of the motions generally match the projection of the magnetic field onto the plane of the sky as predicted by an MHD model of the corona. Some discrepancies between the model and the observation are seen, but they are within the uncertainties of the magnetic field boundary condition at the solar surface employed by the model (Downs et al. 2013). Thus the comet provides a unique way to determine the magnetic 


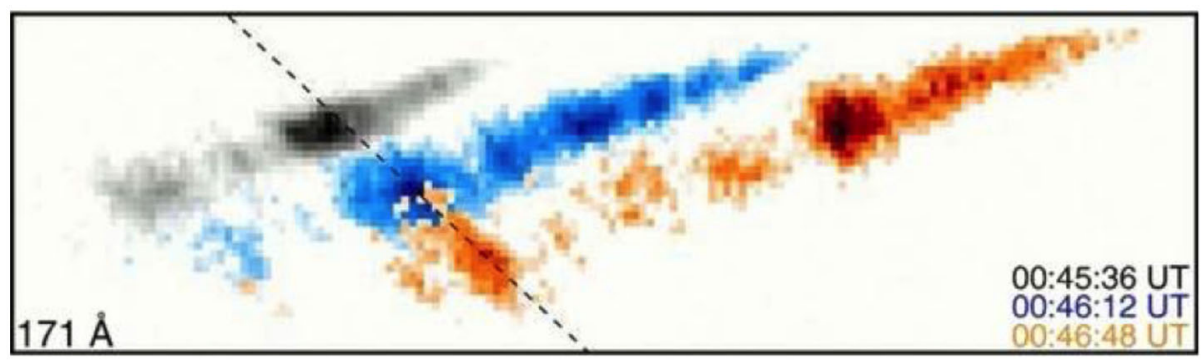

Fig. 24 Superposed AIA images of the comet at three times separated by 36 seconds (black, blue, orange, respectively) (Raymond et al. 2014). The dashed line shows the local direction of the magnetic field, along which striations are aligned. The sequence shows how bright features move along a striation at an angle to the comet's trajectory, whilst also spreading in direction parallel to the magnetic field. The comet is moving almost horizontally across the top of the figure

field structure of the corona a few tenths of a solar radius above the surface with arcsecond positional accuracy.

The very existence of the striations can be used to determine the coronal density. When oxygen atoms are produced by the photodissociation of water, they move through the corona along with the nucleus in a slowly expanding cloud that is invisible to $S D O$-AIA. However, when an atom becomes ionised it behaves as a pickup ion, spiraling along the local magnetic field, and when it reaches the O III to O VI ionisation states it becomes visible in the AIA images (Raymond et al. 2014).

The fact that most oxygen atoms travel through the region between striations without being ionised provides an upper limit on the coronal density there, while the observation that the atoms are ionised in the striation gives a lower limit to that density. The result is that the corona is full of fairly regularly spaced magnetic flux tubes with densities a factor of 6 or more higher than the regions in between, separated by about $4000 \mathrm{~km}$ (Raymond et al. 2014). This density structure implies strong gradients in the Alfvén speed, which in turn implies a turbulent outer scale of a few thousand $\mathrm{km}$ and rapid phase mixing and dissipation of Alfvén waves. The average density required for ionisation of oxygen through the ionisation states observed by the different $S D O$-AIA bands is in line with the predictions of the Downs et al. (2013) MHD model (McCauley et al. 2013; Raymond et al. 2014; Pesnell and Bryans 2014).

\subsection{Magnetic Field}

It is also possible to get some idea of the magnetic field strength. When atoms from the comet are ionised, the component of their velocity parallel to the field becomes the bulk speed along the field, while the perpendicular velocity component becomes a gyro velocity around the field. For neutrals all moving with the comet, the ions form a ring beam in velocity space, which is very unstable. It quickly evolves into a bispherical shell in velocity space (e.g., Williams and Zank 1994; Isenberg and Lee 1996).

The parallel and perpendicular velocities of the pickup ion distribution depend on the component of the magnetic field along the line of sight, as well as that in the plane of the sky. The direction of the striation in the plane of the sky, the speed of the emitting cloud along the striation and its rate of spreading therefore provide a means to determine the relative sizes of all three components. When the velocity distribution evolves from a ring beam to a bispherical shell, some energy is lost to Alfvén waves, and the fraction of energy lost depends upon both the angle between the comet velocity and the field, and upon the ratio 
of the comet speed to the Alfvén speed (Williams and Zank 1994). In principle that gives a means to determine the field strength (Raymond et al. 2014), and the observations of Comet C/2011 W3 Lovejoy are compatible with the MHD model of Downs et al. (2013). However, there is enough uncertainty in the density and enough disturbance of the coronal plasma and field by the cometary material that this is only a rough determination of the coronal field.

The possibility of detecting pickup ions from individual sungrazing comets at $1 \mathrm{AU}$ (215 $\mathrm{R}_{\odot}$ ) from the Sun has been investigated (Iseli et al. 2002). Given that most Kreutz sungrazers are destroyed at perihelion, the pickup ion flux is expected to be much higher at the location aligned with the ascending nodes of these comets' orbits, i.e. pre-perihelion, at a heliocentric distance of $\sim 2.2 \mathrm{R}_{\odot}(0.010 \mathrm{AU})$, than at locations aligned with the postperihelion descending node. The Earth is aligned with the Kreutz group's ascending nodes in September and the first half of October. Pickup ions are therefore most likely to be detected during that period. However, as most sungrazers are very small with tail crossings likely to last less than one hour, the likelihood of the in situ detection of an individual comet's ion tail is small. Bzowski and Królikowska (2005) propose that comets may however be significant contributors to the "Inner Source" of pickup ions detected in the solar wind.

\subsection{Comets' Ion Tails as Solar Wind Tracers}

Comets are natural laboratories for understanding the structure of the solar wind, including the near-Sun region which has not been extensively explored in situ to date. As the appearance, orientation, and dynamics of their ion tails reflect local wind conditions (e.g., Wegmann et al. 1987; Wegmann 2000; Jones and Brandt 2004). Dynamic features are observed in ion tails at all physical scales. Condensations and kinks often propagate in the antisunward direction, some of which have been linked to solar wind features. The general appearance of the tail can also indicate if it is in variable (probably streamer belt) or smooth (probably coronal hole) solar wind flow (Brandt and Snow 2000).

Disconnection Events, DEs, are large-scale removals of significant portions of ion tails that then propagate anti-sunward. It was proposed by Niedner and Brandt (1978) that they were caused by magnetic reconnection when comets cross the heliospheric current sheet, HCS. This scenario has been borne out by combinations of remote observations and solar wind data, though the interplanetary counterparts of CMEs, ICMEs, have been shown to be responsible for some DEs. Even in the case of ICMEs, the cause may ultimately be the same, if magnetic field reversals trigger reconnection (Vourlidas et al. 2007; Jia et al. 2009). Remote observations of comets can therefore be used to constrain the location of the HCS.

Ion tails' orientations are largely controlled by the speed of the solar wind in the vicinity of the comet, and when the observer is well away from the comet's orbital plane, tail orientations can act as an indicator of the wind speed. The radial component of the solar wind flow and the comet's orbital velocity dictate the anti-sunward orientation of the ion tail. The tail lags the prolonged radial anti-sunward vector by an aberration angle of a few degrees. Deviations from a straight tail are visual cues that the comet is encountering variations in the solar wind flow or has encountered a large-scale discontinuity.

It must be noted that there are complications with using ion tail orientations: for reliable speed estimates, it is assumed that the solar wind flow is purely radial, when it is known that is often not the case. In addition, the orientations of the tails determined from in situ and remote observations of near-Sun comets are somewhat unexpected (Jones et al. 2000), generally being at a larger angle to the radial direction than expected. It is likely that this partially results from the large angle between the tail and anti-sunward direction for near-Sun comets. When strongly inclined, regions of solar wind unaffected by the comet's presence 


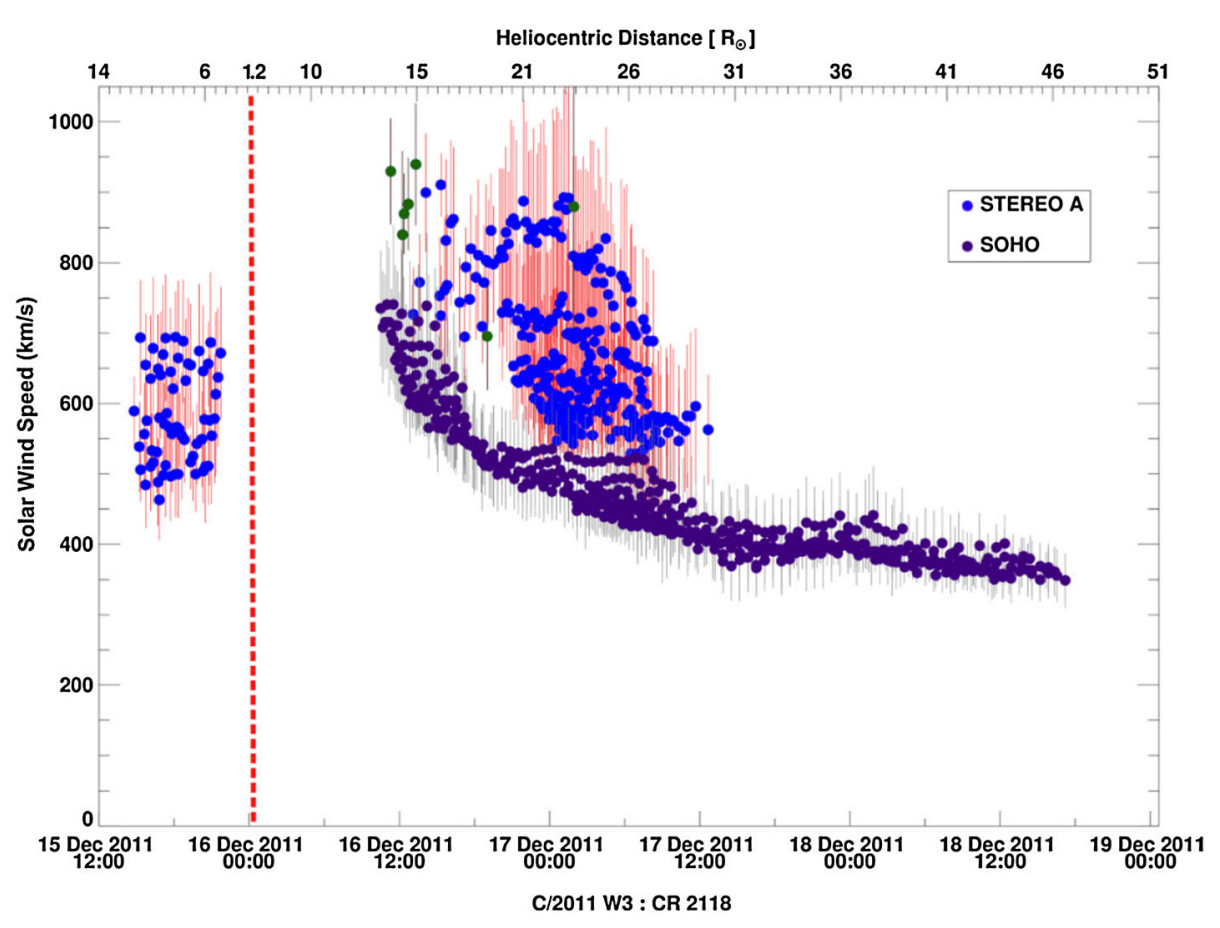

Fig. 25 Solar wind speed estimates derived from the orientation of C/2011 W3's ion tail (Ramanjooloo et al. 2012), and assuming that the solar wind was flowing in a radial direction. The vertical dashed red line indicates the time of perihelion. The discrepancy between results from STEREO and SOHO indicates the presence of a non-radial solar wind flow component

can flow past the tail itself, resulting in the draping of the HMF around the tail. Whether this process further affects the tail's orientation is currently undetermined, however, it is likely to lead to the retention of the magnetotail structure, unlike in hypothetical stationary comet simulations where tail magnetic field signatures eventually decay (Wegmann 2002).

Studies of ion tail orientations have traditionally concentrated on the overall tail orientation (e.g. Brandt and Snow 2000). Recently-developed analysis techniques can instead return a time series of estimated solar wind speeds from a single image (Ramanjooloo 2015). Spaceborne observations of the bright sungrazer C/2011 W3 Lovejoy were gathered by SECCHI aboard STEREO-A and -B and the SOHO-LASCO C2 and C3 coronagraphs during 2011 December 14-19. The comet interacted with a smooth solar wind flow and a gradual transition between the fast and slow solar wind post-perihelion, as evidenced by the featureless ion tail. Comparison of observations from two different vantage points, STEREO-A and SOHO, strongly indicated a non-radial solar wind flow (Fig. 25).

In the most thorough analysis of ion tail orientations carried out to date, Ramanjooloo (2015) concluded that this technique is a good indicator of varying solar wind conditions when the observer is well separated from the comet's orbital plane. However, due to uncertainties in the non-radial flow components, the technique offers limited reliability as a quantitative measure of solar wind speeds. 


\section{Cometary Mass, Momentum, and Energy Loss in the Solar Corona}

\subsection{Introduction}

In this section, we consider the corona-comet interactions that are only significant for sungrazers and sundivers, i.e. comets that are exposed to the most extreme near-solar environment. For any comet that survives to the deep chromosphere, the kinetic energies of cometary material and of the solar plasma become totally dominant over insolation effects. A comet moving at, for example, the solar escape velocity of $619 \mathrm{~km} \mathrm{~s}^{-1}$, is $\sim 12.5 \times$ faster than a thermal proton. The corona is however tenuous, with a maximum base pressure of $0.1 \mathrm{~Pa}$, i.e. a low level laboratory vacuum, or that $\sim 100 \mathrm{~km}$ above Earth's surface. Detailed calculations (Brown et al. 2011, 2015; Schrijver et al. 2012) show that for km-sized bodies, the effects of coronal plasma drag and shock are small, and radiative effects dominate. At the $\sim 140,000 \mathrm{~km}$ minimum altitude (heliocentric distance $0.00555 \mathrm{AU} ; 1.193 \mathrm{R}_{\odot}$ ) at which $\mathrm{C} / 2011 \mathrm{~W} 3$ Lovejoy passed through the corona, the ram pressure was $2 \mathrm{~Pa}$. The main comet nucleus can survive as long as it has water ice and rock to sublimate and cool it. Metre-sized and smaller bodies, however can be dominated by coronal plasma drag and shock, so small fragments detaching from the main comet body are quickly broken up and vaporized.

Plasma drag forces become more significant with decreasing coronal altitude, but radiation pressure likely remains the dominant force acting on dust grains in most regimes where they survive. Comet tails in this extreme environment, after a lag time of $\sim 1 \mathrm{~min}$, have vaporized and warmed up to the local plasma temperature. The $\mathrm{C}, \mathrm{N}, \mathrm{O}, \mathrm{Fe}, \mathrm{Si}, \mathrm{Mg}$, and $\mathrm{S}$ atoms that dominate the comet's composition then begin glowing at wavelengths corresponding to unusually low ionisation states for the corona.

As discussed further below, for perihelion $q \geq 1.01 \mathrm{R}_{\odot}$ (up to a few $\mathrm{R}_{\odot}$ ) and, for likely masses $M_{o}$, a comet's destruction is dominated by relatively gradual sublimation. For $q \leq$ $1.01 \mathrm{R}_{\odot}$, destruction is much faster, $\sim 0.1-10 \mathrm{~s}$ depending on entry angle, as it is dominated by ablative interactions with the very dense lower solar atmosphere. These interactions are accelerated by ram pressure driven pancaking of the nucleus (Brown et al. 2011, 2014). The fates of the mass, momentum and energy lost in the destruction of sungrazing and sundiving comet nuclei and how the fates depend on the orbital and physical properties of the nucleus are of great interest. These are briefly addressed here, and are discussed in greater depth in Brown et al. (in prep.).

\subsection{Comet Nucleus Dynamical Properties}

Cometary nuclei are extremely small compared to the Sun in both volume and mass. Sungrazers are cold objects that move through a solar atmospheric environment that is permeated by an intense $5800 \mathrm{~K}$ photospheric radiation field, a $2 \mathrm{MK}$ kinetic temperature plasma, and a strong solar gravity gradient. There is thus a tendency to think of comets as very fragile objects with little chance of affecting the Sun. However, comets' orbital speeds are highly supersonic, $\sim$ Mach 60 in the chromosphere and Mach 6 in the solar corona, though possibly sub-Alfvénic in strong coronal fields. Their densities $\rho$ of $\sim 500 \mathrm{~kg} \mathrm{~m}^{-3}$ are also extremely high compared to their surroundings: $\sim 10^{7}$ times that in the photosphere and $\sim 10^{14-16}$ times that of the low corona.

Table 7 lists the energy density of a comet's nucleus and various components in the sungrazer/sundiver regime. The combination of high $\rho$ and $v$ implies that the cometary nucleus $\eta_{k i n}$ clearly exceeds the energy density of all other environmental components by a factor of at least $10^{9}$. This implies that the dissipation of nucleus kinetic energy is a key 
Table 7 Energy densities of a sungrazer and various components of its environment

\begin{tabular}{llll}
\hline & Term & Formula & Energy per unit volume $\left(\mathrm{erg} \mathrm{cm}^{-3}\right)$ \\
\hline Nucleus kinetic energy & $\eta_{\text {kin }}$ & $\rho v^{2} / 2$ & $\sim 10^{15}$ \\
Photospheric radiation field & $\eta_{\text {rad }}$ & & $\sim 10$ \\
Photospheric thermal plasma & $\eta_{\text {thPh }}$ & & $\sim 10^{5}$ \\
Low corona thermal plasma & $\eta_{\text {thCor }}$ & & $\sim 3 \times 10^{-2}$ \\
Magnetic field & $\eta_{\text {mag }}$ & $\sim 4 \times 10^{4}$ & $\sim 10^{6}$ (sunspots) \\
& & $\times(B(k G))^{2}$ & $\sim 10^{4}$ (corona) \\
\hline
\end{tabular}

factor in the interaction of the solar atmosphere with the nucleus. The coma and tail densities are much smaller than that of the nucleus, and can be strongly affected by interaction with the solar plasma and magnetic field.

The masses of relatively large $(0.1-1 \mathrm{~km})$ nuclei are $\sim 10^{12-15} \mathrm{~g}$. For comparison, $\sim 10^{12} \mathrm{~g}$ is needed to survive sublimation down to near the photosphere (e.g., Weissman 1983; Brown et al. 2011, 2015). This is roughly the mass range of coronal mass ejections (CMEs) and solar flare ejecta. Their total kinetic energies are $\sim 10^{27-30} \mathrm{erg}$, comparable to the magnetic energy released in small to medium sized CMEs and flares. We note for scale that the mass and energy of a C/1995 O1 Hale-Bopp scale comet at the Sun would be as large as those of the largest CMEs and flares ever observed.

\subsection{Comments on Fragmentation}

Here we consider how the fragmentation of single coherent nuclei accelerates the processes which drive their loss of mass, momentum and energy. This happens through the increases in nucleus material surface area per unit mass that results from break-up.

Although atmospheric ram pressure, volatile outgassing, rotation, etc., can all contribute to fragmentation, tidal fragmentation inside the classical Roche Limit is the most commonly invoked mechanism. This defines the heliocentric distance of $\sim 3.45 \mathrm{R}_{\odot}(0.016 \mathrm{AU})$ at which the solar gravity gradient (tidal) force acting to separate the two halves of a small body in a circular orbit exceeds the mutual gravitational force keeping them together (Eq. (1)). On the basis of this figure, and using our proposed definition of a sungrazer, it is commonly predicted that sungrazers will fragment, but this is an oversimplification for several reasons:

1. The point $r=\mathrm{R}_{\text {Roche }}$ for a circular orbit is that where self-gravity and tidal forces just balance. Consequently for an orbit of radius $\mathrm{R}_{\text {Roche }}$, the tidal disruption time becomes small compared to the orbital period. On a parabolic orbit with $q$ just inside $\mathrm{R}_{\text {Roche }}$, only a short period is spent inside $\mathrm{R}_{\text {Roche }}$. The effective tidal disruption distance for parabolic or hyperbolic (stray) orbits is therefore $<\mathrm{R}_{\text {Roche }}$. The importance of how the stray and circular cases differ is commonly ignored, though it has long been discussed (e.g., Dobrovolskis 1990; Boss et al. 1991; Davidsson 1999, 2001; Holsapple and Michel 2006; Toth and Lisse 2006; Veras et al. 2014 and references therein). In estimating the reduction in limiting distance, the higher orbital speed (and centripetal acceleration) in the stray cases also has to be taken into account. Care should be taken to recognize that tidal disruption is less relevant to the rapid flyby of sungrazers than to near-circular orbits.

2. In the case of sundivers, the impact ablative destruction time is extremely short $\sim H / v_{o}$ (see below), meaning that the effect of tidal disruption may be small unless the tidal disruption has progressed significantly before the onset of the very rapid ablative destruction. 
3. The basic Roche limit result (Sect. 1.3) ignores the nucleus material strength, which, though small, reduces $\mathrm{R}_{\text {Roche }}$. On the other hand, nucleus rotation tends to increase it, e.g., Davidsson (2001) as do interior pressure forces from outflowing volatiles. It has been suggested that there is a significant confining pressure from the surface outflow which can actually inhibit fragmentation (Gundlach et al. 2012). We note that $C / 2011$ W3 Lovejoy possibly survived perihelion passage well inside the Roche limit with only partial fragmentation (Sekanina and Chodas 2012).

Brown et al. (2015) have pointed out that, at least for sundivers, the nucleus behavior is a function of the ratio of the column mass $\left(\mathrm{g} \mathrm{cm}^{-2}\right)$ of the nucleus relative to that of the atmosphere at the atmospheric column mass depth considered. If a nucleus fragments into $F$ equal objects, the column mass of each is $F^{-1 / 3}$ times that of the whole body. Hence the column density in the atmosphere at which a fragment evolution stage is reached, e.g. total ablation, is smaller by a factor $F^{1 / 3}$ than that for the whole nucleus.

\subsection{The Case of Sundivers}

Sundivers of sufficiently high initial mass and small enough perihelion distance will have lost little of their mass by insolative sublimation before they reach the atmospheric region where destruction results from fluid/collisional interactions rather than by insolation (Brown et al. 2015). The latter destruction mode requires an atmospheric density so high that ram-pressure driven distortion (pancaking) and deceleration, and bow-shock driven ablative mass loss, occur much faster than insolative sublimation, thermal conduction from the atmosphere, or other processes.

Sundivers of low mass will sublimate fully before reaching these high densities. Higher mass objects must be rare but are potentially of great interest as a means of studying nucleus properties from the depths they reach and the spectra they emit as they undergo abrupt explosive destruction (cf. Brown et al. 2011, 2015). They are likely to be much more common in some stellar environments such as young stars and White Dwarfs (e.g., Alcock et al. 1986; de Winter et al. 1999; Veras et al. 2014).

In the case of large body impacts in planetary atmospheres it is usually argued (e.g., Chyba et al. 1993; Mac Low and Zahnle 1994) that deceleration dominates, greatly slowing the body before significant ablative mass-loss occurs. In this regime, the comet kinetic energy and momentum are deposited mainly into the atmosphere, as in the case of a satellite re-entry heat shield. Brown et al. (in prep.) consider the deposition of a comet's kinetic energy and momentum into the solar atmosphere, and conclude that in the solar case nucleus termination is most likely ablative mass-loss dominated, because of the much higher impact speed (cf. Brown et al. 2015).

The sundiver's total kinetic energy of $2 \times 10^{30} M_{15} \operatorname{erg}\left(M_{15}=M_{o} / 10^{15}\right)$ is deposited within about one scale height $H$ of $\sim 200 \mathrm{~km}$ vertically or about $10^{23} \mathrm{erg} \mathrm{cm}^{-1}$ over a very small area. Such a highly impulsive localised release of energy and momentum into the solar atmosphere will produce flare-like phenomena including a hot rising plume of high metallicity, and helioseismic ripples in the photosphere. Hot plume spectroscopy of such an event should give a good indication of whole comet abundances rather than just surface values (Brown et al. 2011, 2015). A quantitative treatment of the explosion, helioseismic effects and spectroscopy requires full numerical simulations.

\subsection{The Case of Low Perihelion Sungrazers}

The theory of low $q$ sungrazer destruction, where perihelion occurs just above the solar photosphere such that the trajectory is not as sharply inclined to the solar surface as that of 
a sundiver, is in some respects more complicated than the bolide-like physics of the latter objects. In particular, for sundivers, collisional hydrodynamic interactions with the dense chromosphere, such as ram pressure and ablation processes at the bow shock, dominate the nucleus mass loss. Insolative sublimation is negligible in comparison. These processes also dominate the conversion of nucleus kinetic energy and momentum into the heating of, and bulk motion and wave generation in, the solar atmosphere and ablated (high metallicity) cometary material intermingling with it. These processes occur much more quickly than tidal fragmentation.

For comets less than $\sim 2 \mathrm{R}_{\odot}(0.0092 \mathrm{AU})$ from the Sun's centre, the typical rise time of the sublimative mass-loss rate is $\sim \mathrm{R}_{\odot} / v_{o}$. This is also the timescale for the destruction of a sungrazer with a mass just high enough to reach perihelion $q \sim \mathrm{R}_{\odot}$. According to Brown et al. (2011) (their equation (27)) the minimum mass surviving sublimation to perihelion $q$, without fragmentation, is $\sim 10^{11} \mathrm{~g}\left(q / \mathrm{R}_{\odot}\right)^{3 / 2}$.

Comet C/2011 N3 SOHO vaporized completely, close to its perihelion, so had a lifetime in the Sun's atmosphere of $\sim \mathrm{R}_{\odot} / v_{o}$. This is long enough for tidal disruption to be important. For example (cf. Schrijver et al. 2012) the mass loss rate from C/2011 N3 near its end-point was higher than that expected from the $10^{11} \mathrm{~g}$ incident mass which would end there in the unfragmented case (Brown et al. 2011). Moreover, a mass loss rate an order of magnitude larger than that of Schrijver and co-workers is needed to explain the brightness seen by SDO-AIA according to the theory of Bryans and Pesnell (2012) and McCauley et al. (2013). Hence C/2011 N3 was most likely a higher mass $\left(\sim 10^{12} \mathrm{~g}\right)$ object which had fragmented, thus boosting its sublimation rate. The much more massive nucleus of $\mathrm{C} / 2011 \mathrm{~W} 3$ Lovejoy ( $M \sim 10^{14-15} \mathrm{~g}$ ) was not fully vaporized as it passed through perihelion. The fractional sublimative mass loss predicted by Brown et al. (2011) equation (27) for this mass range and perihelion value being $\sim 2-4 \%$.

The sublimative mass-loss is driven by the $5800 \mathrm{~K}$ photospheric radiation field, which delivers a much higher energy flux $\left(\sim 6 \times 10^{10} \mathrm{erg} \mathrm{cm}^{-2} \mathrm{~s}^{-1}\right)$ than the collisional/shock interaction between the nucleus and the tenuous corona $\left(2 \times 10^{7} \mathrm{erg} \mathrm{cm}^{-2} \times n / 10^{8} \mathrm{~cm}^{-3}\right)$, or the conductive flux from the hot $(2 \mathrm{MK})$ corona itself. The latter is also a few $\times 10^{7} \mathrm{erg} \mathrm{cm}^{-2} \times\left(n / 10^{8} \mathrm{~cm}^{-3}\right)$ even for saturated heat flux. However, sunlight cannot heat the cometary tail material to its observed EUV temperatures, nor can it deliver significant momentum. Thus any substantial loss of kinetic energy and momentum from the cometary mass has to occur by the deceleration of the ablated mass into the tail, through interactions with the solar atmosphere and its magnetic field.

The column mass density $S_{\text {cor }}$ along a trajectory through the low corona is roughly $n m_{p} \mathrm{R}_{\odot} \sim 10^{-5} n_{8} \mathrm{~g} \mathrm{~cm}^{-2}$ (with $n_{8}=n / 10^{8}$ ). Momentum sharing by drag interaction between this and sublimated cometary material is capable of substantially decelerating such material with a comparable column mass. The coronal magnetic field $B$ enhances the deceleration (by a factor of order the ratio of magnetic pressure to atmospheric ram pressure $\left.1 / b_{v}=B^{2} /\left(4 p_{p m} v_{o}^{2}\right) \sim 0.5 B^{2} / n_{8}\right)$ so any $B \gg 1 \mathrm{G}$ will dominate over hydrodynamic forces and would be capable of decelerating a cometary column mass $\sim 5 \times 10^{-4} \mathrm{~g} \mathrm{~cm}^{-2}$.

The column mass of a comet of mass $M$ is $S_{n u c} \sim 10^{5} M_{15}^{2 / 3} \mathrm{~g} \mathrm{~cm}^{-2}$ which, even for the lowest mass close sungrazers, is much too big for the atmosphere or magnetic field to affect the nucleus's motion, unless the nucleus fragments greatly (cf. Sekanina and Kracht 2015). On the other hand, the column density of sublimated material falls greatly as it expands radially. For nucleus masses $M \gg 10^{11} \mathrm{~g}$, equation (24) of Brown et al. (2011) gives the mass $M_{s}$ sublimated before perihelion (in the absence of fragmentation) as a function of $M$ and $q$. For $q \sim \mathrm{R}_{\odot}$ this gives $M_{s} \sim 8 \times 10^{11} \mathrm{~g}$ for $M=10^{12} \mathrm{~g}$. For this tail material to be greatly decelerated by atmospheric drag alone within a distance $\mathrm{R}_{\odot}$, it would need to spread 


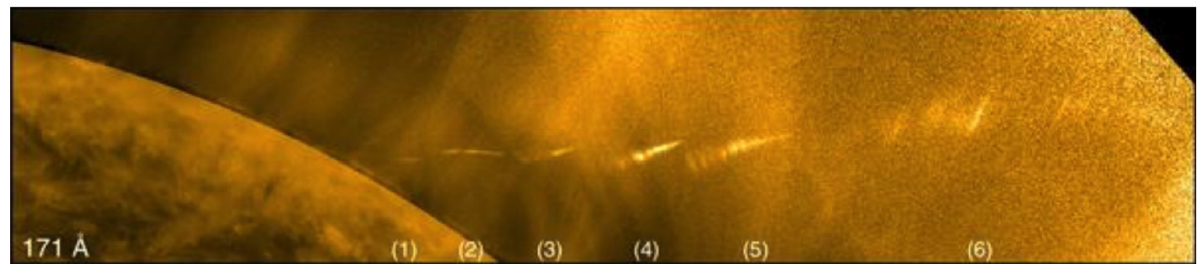

Fig. 26 Composite of six images in the SDO-AIA $171 \AA$ band (McCauley et al. 2013). Striations appear at an angle to the comet's path, sometimes above and sometimes below its trajectory depending on the direction of the magnetic field. The comet's motion was horizontal in this view, from left to right

over a lateral area $\sim M_{s} 10^{17} \mathrm{~cm}^{2} / n_{8}$ or a tail/coma thickness of $\sim 3000 \mathrm{~km} / n_{8}^{1 / 2}$. This is less than that of $\mathrm{C} / 2011 \mathrm{~N} 3 \mathrm{SOHO}$ while, for the magnetic pressure dominated regime, this would be reduced to $\sim 4000 \mathrm{~km} / \mathrm{B}$. The mass sublimated from low mass close sungrazers can thus be fully decelerated by the corona, even with no magnetic field. On the other hand, for $M=10^{15} \mathrm{~g}$, Brown et al. (2011) give $M_{s} \sim 10^{14} \mathrm{~g}$ requiring expansion to a lateral area $\sim 10^{17} \mathrm{~cm}^{2} / n_{8}$ or a tail/coma thickness of $\sim 30,000 \mathrm{~km} n_{8}^{1 / 2}$ or $\sim 40,000 \mathrm{~km} / \mathrm{B}$. Thus, for larger comets with masses $\sim \mathrm{C} / 2011 \mathrm{~W} 3$ Lovejoy, the coronal mass and field are just about able to fully decelerate the sublimated mass in the tail which is a small fraction of the original nucleus mass.

These predictions are consistent with what was seen in the case of C/2011 W3 Lovejoy. Striations were observed at the comet, following the local B field (Fig. 26). These featuresof a different nature to the dust tail striae discussed in Sect. 9.1-show that the motion of the gas produced by the comet was dominated by the magnetic field (McCauley et al. 2013; Downs et al. 2013; Raymond et al. 2014). For even larger comet masses, the gas produced by the comet would strongly distort the magnetic field without being significantly decelerated.

Both sundivers and comets far from the Sun drive bow shocks in the ambient plasma. Close sungrazers move in a plasma of much higher Alfvén speed $\left[v_{A}(\mathrm{~km} / \mathrm{s}) \sim 300 \times\right.$ $\left.B /\left(10^{2} \mathrm{G}\right) /\left(n / 10^{8} \mathrm{~cm}^{-3}\right) \mathrm{km} / \mathrm{s}\right]$, in cases where $B /\left(10^{2} \mathrm{G}\right) /\left(n / 10^{8} \mathrm{~cm}^{-3}\right) \gg 2$, there is no bow shock. The low coronal density also results in a long coronal mean free path, namely around $100 \mathrm{~km} / n_{8}$ for interaction of $600 \mathrm{~km} \mathrm{~s}^{-1}(2 \mathrm{keV})$ protons with cold electrons. This is much larger than the largest cometary nuclei. The much smaller Larmor radius may be the more relevant length scale for comparison.

\subsection{Sources of Energy for the Hot Tail XUV Emission}

Temperatures of $10^{6}$ are needed to generate the cometary tail XUV emission discovered by Schrijver et al. (2012) using SDO-AIA in C/2011 N3 SOHO and C/2011 W3 Lovejoy (Fig. 26), though not seen from Comet C/2012 S1 ISON. The source of this energy and how it heats/energises the tail XUV emission are key questions to be answered concerning close sungrazers.

Possible energy sources for a hot tail and SDO-AIA tail emission include:

1. A persistent, hot ( $2 \mathrm{MK})$ coronal plasma electron component. This is assumed to be entrained in the comet tail in the valuable XUV emission modeling carried out by Bryans and Pesnell (2012) and Pesnell and Bryans (2014). Based on this assumption, they made predictions of the evolution of ionisation state and emission line intensity which broadly agree with the $S D O$-AIA data for plausible cometary abundances. The complementary issues of how the plasma became entrained and kept hot are addressed in Sect. 11.7. 
2. Kinetic energy conversion accompanying the deceleration of sublimated mass that forms the tail. Brown et al. (2015) have suggested this instead as the most likely source, since the kinetic energy lost has to go somewhere. The mean initial kinetic energy per nucleon in this decelerating sublimated matter is $\sim 2 \mathrm{keV}$. They argue that only a few $\%$ of the energy released going into heating high metallicity tail material would provide the near MK temperatures which SDO-AIA data require in the tail. As presented above, atmospheric drag and magnetic forces can fully decelerate small comets like C/2011 N3 $\mathrm{SOHO}$, the entire mass of which is sublimated in the solar atmosphere. Larger comets like C/2011 W3 Lovejoy only undergo partial sublimation, but atmospheric drag and magnetic forces can substantially decelerate the sublimated mass. The sublimation tails of still larger comets are much too massive to be fully decelerated by these forces but will still release some of their kinetic energy into tail heating.

3. Release of magnetic energy of a (non-potential) coronal field. This can be higher than the coronal thermal energy since the corona is a low- $\beta$ plasma at the altitudes under consideration $\left(\beta=n k T /\left(B^{2} / 8 \pi\right)=3 \times 10^{-4} n_{8} T_{6} / B_{2}^{2}\right)$. However, it is unclear how much energy can be released or how efficiently it can be transported into the cometary plasma.

For all three possible sources, the heating process has to be able to compete with radiative cooling, and the energy must be transferred to the electrons, as discussed below.

\subsection{Coronal Electron Heating}

Bryans and Pesnell (2012) and Pesnell and Bryans (2014) assumed that coma and tail ionisation are dominated by collisions with free coronal electrons. They further assumed the persistent presence of such hot coronal electrons in the body of the tail/coma throughout the event and did not consider radiative cooling. We now consider the origin of these free electrons and how their high temperatures can be sustained while they heat and ionise the much denser cometary material.

The mean free path of hot electrons is $\lambda_{e}(\mathrm{~cm}) \sim 2 \times 10^{7} T_{6}^{2} / n_{8}$. In the corona, this is only $\sim 10^{8} \mathrm{~cm}$ and in the cooler denser comet tail is smaller still, much less than the tail thickness. This, combined with the very small Larmor radius in the presence of a strong tail magnetic field, means that as the sublimated tail mass propagates and slows, it does not entrain hot coronal electrons but rather sweeps them aside. Consequently any heating and ionisation of tail material by coronal electrons can only occur by diffusive electron heat conduction from outside the tail. It has to be asked whether this process is rapid enough to overcome the radiative losses and to keep the tail hot during its observed lifetime.

The energy needed to heat the EUV emitting tail to $\sim 10^{6} \mathrm{~K}$, which was the temperature indicated by the relative count rates in the SDO-AIA bands (McCauley et al. 2013) can be estimated. Much of a nucleus of mass $M \sim 10^{12} \mathrm{~g}$ is vaporized so $M_{s} \sim 10^{12} \mathrm{~g}$ and the energy required is $\sim 10^{25} \mathrm{erg}$. This energy can be related to the volume $V_{\text {cor }}$ of the 2 MK corona containing that amount of thermal energy at density $n_{c o r}=10^{8} n_{8} \mathrm{~cm}^{-3}$, namely $V_{\text {cor }} \sim 3 \times 10^{26} / n_{8}$. Over a tail length $L=10^{10} L_{10} \mathrm{~cm}$, heat then has to be drawn from a coronal cylinder of diameter $d \sim\left(V_{\text {cor }} / L\right)^{1 / 2}$ which is $d \sim 0.7 \times 10^{8} \mathrm{~cm} /\left(n_{8} L_{10}\right)^{1 / 2}$ for $M \sim 10^{12} \mathrm{~g}$. This is roughly comparable with or smaller than observed tail thicknesses. 2 MK thermal electrons with thermal speeds $\sim 10^{9} \mathrm{~cm} / \mathrm{s}$ can travel these distances in under $1 \mathrm{~s}$ but can carry a maximum (saturated) heat flux $F_{\text {sat }} \sim 10^{8} n_{8} \mathrm{erg} / \mathrm{cm}^{2} / \mathrm{s}$ and a total conductive power $\sim \pi L d F_{\text {sat }} \sim 5 \times 10^{26} n_{8} \mathrm{erg} / \mathrm{s}$ and $5 \times 10^{27} n_{8} \mathrm{erg} / \mathrm{s}$, respectively. This would deliver the necessary heating energy in $\lesssim 0.1 \mathrm{~s}$ in both cases, provided that they penetrate the cometary gas and that radiative cooling does not prevent it. 
The radiative cooling time of hot plasma is given by

$$
\tau_{\text {rad }}(s) \sim 3 n k T / n^{2} f_{\text {rad }}(T)=3 k T / n f_{\text {rad }}(T)
$$

where $f_{\text {rad }}(T)$ is the radiative loss function (Cox and Tucker 1969). For MK coronal plasma, $f_{\text {rad }}(T)$ has a maximum value $\sim 10^{-21} \mathrm{erg} \mathrm{cm}^{3} \mathrm{~s}^{-1}$ so that $\tau_{\text {rad }}(s) \sim 4000 \times T_{6} / n_{8} f_{-21}$ where $T_{6}=T / 10^{6} \mathrm{~K}, n_{8}=n / 10^{8} \mathrm{~cm}^{-3}$, and with $f_{-21}=f_{\text {rad }} / 10^{-21} \mathrm{erg} \mathrm{cm}^{3} \mathrm{~s}^{-1}$. So, for coronal density $n_{8} \sim 1$ at a close sungrazer height, $\tau_{\text {rad }} \sim 4000 \mathrm{~s}$. This is much longer than the conductive heating time and roughly comparable to the observed lifetimes of close sungrazer tails. However, not only is the comet tail density much higher than that of the corona (which alone reduces $\tau_{\text {rad }}$ by a factor $\sim n_{\text {cor }} / n_{\text {com }}$ ) but the cometary matter has much higher metallicity. Because the $\mathrm{O}: \mathrm{H}$ ratio in a comet is $\sim 0.5$, rather than the 0.0005 of the corona, $f_{\text {rad }}$ is increased by $\sim 500 \times$, implying a tail radiative cooling time of $8 \mathrm{~s} \times$ $\left(n_{\text {cor }} / n_{\text {com }}\right)$. This could be much shorter than the conductive heating times discussed above.

In their SDO-AIA modelling, Bryans and Pesnell (2012) mention an upper limit $n_{\text {com }} \lesssim 10^{11} \mathrm{~cm}^{-3}$ presumably for the densest, most emissive, tail regions. On the other hand, we note that a value $n_{\text {com }} \sim 10^{8} \mathrm{~cm}^{-3}$ is implied by the $S D O$-AIA striations from the ionisation lengths (times) of the ions, but this is far down the tail. Such times would be much too short to agree with tail emission lifetimes unless offset by a power input more intense than saturated coronal electron conduction. Moreover, the coronal electrons are tied to the coronal magnetic field, which is thought more likely to wrap around the cometary plasma than to penetrate it, e.g. Jia et al. (2014). These arguments appear to suggests that coronal electrons are unlikely to supply all the heat that generated the EUV emission observed by SDO-AIA.

\subsection{Collisional Thickness Considerations concerning Tail Heating and Ionisation}

In discussing the ionisation and excitation of the cometary material giving rise to the EUV/XUV emission seen by SDO-AIA, Bryans and Pesnell (2012) discussed various competing collisional mechanisms, plus, for lower ionisation stages, photoionisation by solar optical and UV radiation. These collisional mechanisms included: impact ionisation and the excitation of cometary neutrals by impinging coronal protons, charge exchange with cometary neutrals, and impact ionisation and excitation by coronal thermal electrons. The effectiveness of these processes were compared solely in terms of local thin target volumetric rates, namely $R\left(\mathrm{~cm}^{-3} \mathrm{~s}^{-1}\right)=F \xi n$, where $F$ is the flux of incident particles and $\xi$ the cross section for the collisional interaction process under consideration.

Bryans and Pesnell concluded on this basis that the dominant ionisation process is by coronal thermal electron impacts because their number flux $F_{e}=n_{\text {cor }} v_{e}=7 \times 10^{16} n_{\text {cor }} T_{\text {cor } 6}^{1 / 2}$ is about 15 times higher (since $v_{e} \sim 10 v_{o}$ ) than that of atmospheric protons bombarding the comet, namely $n_{\text {cor }} v_{o}=6 \times 10^{15} n_{\text {cor } 8}$. Their energy fluxes however are quite similar since proton impact energies $m_{p} v_{o}^{2} / 2 \sim 2 \mathrm{keV}$ are about 10 times higher than $m_{e} v_{e}^{2} / 2 \sim 0.2 \mathrm{keV}$. In a thin target, i.e. one across which the incident particle speed only drops slightly, the total rate of the process $\left(\mathrm{s}^{-1}\right)$ is simply $F \xi n$ times the whole source volume. If, however, the target is collisionally thick enough to stop the incident particles then the total rate of any process is fixed by the particle injection rate times the number of times the process occurs along the entire stopping path of each incident particle, independent of the target column density, if high enough (Brown 1971). The thick target column densities $N(=1 / \xi)$ for a $2 \mathrm{keV}$ proton undergoing Coulomb or neutral $\mathrm{H}$ collisions are both of order $10^{15} \mathrm{~cm}^{-2}$ (Emslie 1978); i.e., $\xi$ for these processes are around $10^{-15} \mathrm{~cm}^{2}$. 
It was noted above that the stopping $N=n \lambda_{e}$ for a coronal thermal electron is similar in value. For charge exchange interactions, Bryans and Pesnell (2012) (Sect. 4.2.1) quote $\xi \sim 6 \times 10^{-15} \mathrm{~cm}^{-2}$. All of these cross sections are roughly comparable and imply that, at a cometary tail density $n_{\text {com }}=10^{11} n_{11} \mathrm{~cm}^{-3}$, all of the processes are 'complete' within $\sim 0.1 \mathrm{~km} / n_{11}$ of entering the cometary matter. For any plausible $n_{\text {com }}$, this is a very thin layer compared to the dimensions of the tail. Penetration of electrons and ions is further restricted in the presence of a longitudinal field by their small Larmor radii. This means that the entire energy fluxes of both coronal thermal electrons and bombarding protons are deposited in thin surface layers. In the case of the hot coronal electron (conductive) flux, the thin surface layer heated by it is along the tail's longitudinal surface. This will not drive ionisation uniformly through the cometary matter, but rather cause intense heating and ionisation of the small mass in that layer.

\subsection{Coronal Proton Bombardment as the Source of Comet Tail Heating}

The situation for heating and ionisation by atmospheric proton bombardment is geometrically and physically different. As pointed out by Bryans and Pesnell (2012) coronal protons cannot directly ionise the gas from the comet, but they might provide heat to the electrons. In principle, tail heating by the atmospheric protons bombarding the comet's head is a more likely cause than coronal electrons, so they are a more likely cause for the driving of the EUV emission seen by SDO-AIA. In broad energetic terms, it requires $433 \mathrm{eV}$ to ionise $\mathrm{O}$ to $\mathrm{O}^{6+}$, as observed in Comet $\mathrm{C} / 2011 \mathrm{~W} 3$ Lovejoy (McCauley et al. 2013). While the atom is being ionised, it will also be excited, producing the observed EUV lines as well as more intense longer wavelength lines. Following McCauley and co-workers' method, the total energy in these lines is about $1500 \mathrm{eV}$ per $\mathrm{O}$ atom. Since the coronal protons carry $2 \mathrm{keV}$ each when they encounter the comet, and since momentum conservation suggests that $\sim 16$ coronal protons are needed to decelerate each $\mathrm{O}$ atom from the comet, there is enough energy available. However, if the cometary plasma is decelerated by the magnetic field rather than the mass of the coronal gas, far fewer coronal protons are needed. Raymond et al. (2014) found no evidence for deceleration of the material moving along the field in the striations of Comet C/2011 W3 Lovejoy, indicating that the cometary gas was not decelerated by collisions with coronal plasma. Further uncertainties arise over how well the coronal protons mix with the cometary gas given their possible confinement by the magnetic field.

\subsection{Heating by Cometary Ions}

Under some conditions, when atoms leave the coma they travel with the comet until they become ionised, at which point they become pickup ions. Their velocity component along the field direction is conserved, while the perpendicular component is first isotropized into a shell in velocity space, then converted to a thermal velocity distribution, e.g. Williams and Zank (1994). Since each O atom carries $32 \mathrm{keV}$, there is plenty of energy available to ionise the atoms and balance radiative losses, and there is no need to transport energy from the corona into the cometary plasma. There remains the question of transferring energy from the ions to the electrons. A rough estimate suggests that Coulomb collisions are adequate to heat the electrons, but a more detailed calculation is needed. An interesting possibility is that a few ionisations initiate runaway ionisation, like the charge exchange avalanche proposed by Gombosi (1987), and that this generates the pickup ions and hot electrons to sustain the ionisation and emission observed by $S D O$-AIA. 


\section{Exocomets}

Having discussed many aspects of near-Sun comets, we now consider the related research topic of comets near other stars. There is now extensive evidence of near-star bodies orbiting $\beta$ Pictoris, $\beta$ Pic, a young, massive circumstellar disk of $\sim 10 \mathrm{MYr}$ in age. Exocomets are revealed from transient CaII absorption features seen almost continuously in high-resolution optical spectra plus other heavy species observed in the UV. These are presumed to arise from the sublimation of Ca-bearing silicates released from individual objects, creating significant absorption when they pass in front of the primary star along our line of sight. An important aspect of this system is that the high luminosity of this A-type star relative to the Sun changes the sublimation distance for a given material compared to our solar system by a factor 3, significantly increasing the sublimation rates for exocomets near the star. Redshifted (infalling) absorption is much more common than blueshifted absorption, allowing modelling of the scattering and dynamical evolution of these bodies. Recently, analysis of $\sim 6,000$ transient features over an 8 year period allowed identification of two distinct dynamical families, one of which may be controlled by a mean motion resonance with the directly imaged giant planet (Kiefer et al. 2014).

Several other A-stars, which are, by definition, all young, have been found to exhibit anomalous CaII absorption lines variable in wavelength and time, which by analogy with $\beta$ Pic are also believed to be associated with infalling exocomets near their parent star, sometimes termed Falling Evaporating Bodies, FEBs (e.g., Montgomery and Welsh 2012; Welsh and Montgomery 2013; Kiefer et al. 2014). In some systems, Na I absorption is also seen, presumably produced in the same manner as the sodium tails of comets and sungrazers in our solar system. The large number of A-type stars with detectable warm inner system dust ( $\sim 10 \%$, as opposed to $\sim 2 \%$ rate for FGK stars), is likely due to a combination of low stellar age, massive and active circumplanetary disks, and high rates of stellar insolation. Because these systems are young and "dynamically warm," i.e. still have high rates of important planet crossing, resonant, and collisional interactions, we can expect the processes that define the orbits of Near-Sun comets in the Solar System to be particularly active.

Still to be found is evidence for exocomets around more Sun-like FGK stars. The current limitations on doing this are the relative faintness of the primary star and smaller size and mass of the protoplanetary/circumstellar disk. When exocomets are common, the star is typically in the T Tauri phase, and the primary star is obscured enough that individual cometary passages are difficult to detect. The future advent of larger and more sensitive telescopes performing high-resolution spectroscopic surveys on Class III young stellar objects and weak-line T Tauri stars should hopefully help improve this situation.

At the other end of solar system evolution, we now have evidence for near-star objects in very old systems whose primary stars have evolved away from the main sequence. Around $5-10 \%$ of DZA and DZB white dwarfs, the expected end point of our Sun's evolution, have atmospheres "polluted" by infalling silicaceous dust derived from small bodies orbiting near the $\sim$ Earth sized white dwarfs. The presence of heavy elements cannot have arisen from the interstellar medium, which is too rarefied and hydrogen-rich. Rather they must originate from planetary system remnants (e.g., Gänsicke et al. 2016).

The composition of these asteroids and comet nuclei resembles that of the bulk Earth with $85 \%$ (by mass) composed of $\mathrm{O}, \mathrm{Mg}, \mathrm{Si}$, and $\mathrm{Fe}$. There are also trace amounts of $\mathrm{C}$, and evidence has also been found for water-rich material (Farihi et al. 2013). These bodies have survived the transition of the primary star to a white dwarf, and some of them have approached close enough to the star to be disrupted and form a very close-in $(\sim 0.1 \mathrm{AU})$ radius debris/accretion disk. Dynamical simulations have shown that both grazing encounters (Veras and Gänsicke 2015) and direct stellar impacts (Veras et al. 2016) should occur. 
In some of these cases the surface contamination may be delivered via the slow infall of a dense accretion disk of which around 40 have been observed orbiting within the approximate Roche limit for white dwarf (WD) stars (e.g., Manser et al. 2016). Details of the processes destroying infalling bodies have been modelled by Brown et al. (2017), whose conclusions include the fact that the sizes of (partially sublimated) bodies actually arriving near the WD surface are limited by the strong tidal forces to around $1 \mathrm{~km}$ for strong rock and to a few metres for a typical comet; much smaller than for the solar case.

Another extraordinary elderly "near-star" system is IRC+10-216 (CW Leonis), the brightest infrared (IR) stellar source known. In 2001 the SWAS satellite observed this system and determined that it contains a highly luminous AGB star in the throes of losing its outer stellar envelopes and evolving into a white dwarf (Melnick et al. 2001). In the process of doing this, it is evaporating its own Kuiper Belt. This is a case of near-star comets-except that the star is approaching the comets, not the comets approaching the star!

\section{Outlook}

The study of near-Sun comets helps to expand our knowledge of comets as a whole, as well as to probe the near-Sun environment. Our knowledge of these objects has undergone a revolution since the launch of space-based observatories dedicated to monitoring the Sun and the inner heliosphere. The $\mathrm{SOHO}$ spacecraft in particular has been a primary driver in this breakthrough.

\subsection{Outstanding Scientific Questions}

There are numerous aspects of near-Sun comets that we do not currently fully understand. Some of the key questions to be addressed include the following:

- Do comets that have passed close to the Sun retain volatiles? If so, how deeply are those volatiles buried?

- How many of the sungrazers are actually objects that would be classified as asteroids when further from the Sun? If a sufficient number of these objects can be studied, insights may be gained into evolutionary effects produced by repeated close approaches to the Sun, e.g., broadband colors, phase function, and albedo (if combined with IR observations).

- Why do many objects break up significantly before tidal forces dominate? Is there a single dominant mechanism?

- Are there populations of near-Sun comets we don't yet know? If so, is it because they are too small for detection, or that they have an unfavorable geometry for discovery with current facilities? Could some objects already known about belong to families whose other members have not yet been observed?

- Are sungrazers indeed significant contributors to the inner source of solar wind pickup ions?

\subsection{Future Studies Required}

In order to maximize the scientific return from each comet's perihelion passage by preplanning dedicated observations, rapid post-discovery orbit calculation and dissemination is needed, in particular for sungrazers. Ideally, a regular survey is needed to find new sungrazers well before perihelion. Kreutz sungrazers benefit from having modestly well-constrained 
orbital parameters. Existing optical/IR surveys have not found anything, nor have the best dedicated (short term) searches (Ye et al. 2014), so these comets may be much less active than previously assumed.

For such a search, the larger the solar elongation of the search region, the deeper each exposure needs to be. At large elongations and hence low target brightnesses, trailing becomes problematic so it probably has to be carried out by a telescope of large aperture, such as The Large Synoptic Survey Telescope, LSST (see Sect. 13.3.2). Small solar elongations benefit from brighter search targets, but have a brighter sky background. If no pre-perihelion Kreutz sungrazers continue to be found in the LSST era, then we may have to revise our assumptions about how "cometary," i.e. volatile-rich, these objects actually are compared to the larger members of that group. In addition, we encourage the community to pursue a comprehensive study of $\mathrm{SOHO}$ Kreutz comets' morphology, accounting for seasonal trends and orbital/viewing geometry to fully explain and hopefully better understand the diversity we observe in this group.

In addition to the clear value for solar physics, heliophysics, and space weather applications, continuous coverage of the region surrounding the Sun by a coronagraph is important for the advancement of our understanding of near-Sun comets. We advocate a direct successor to $\mathrm{SOHO}$-LASCO, i.e. an instrument that provides an uninterrupted view of the Sun with white light coronagraphs. A higher spatial resolution than $\mathrm{SOHO}$-LASCO can provide would be beneficial for morphological studies. Several colour bandpass filters would be ideal, plus the capability for polarization observations. The primary, "clear" bandpass would ideally cover sodium D-line emission, and the spatial coverage would at least include the region where Kreutz groups objects are brightest, $\sim 5-20 \mathrm{R}_{\odot}(0.02-0.09$ AU). An instrument that covers a wider region all the way around the Sun, i.e. an extended version of STEREO-SECCHI HI-1, would be ideal for the early detection of inbound sungrazers and the monitoring of all near-Sun comets. Data should be transmitted to Earth as early as possible, to allow as early a detection of new comets as soon as possible, to facilitate dedicated observations. Spectroscopic capabilities, such as those of SOHO-UVCS and also at optical wavelengths, would be particularly beneficial. Solar Orbiter and Parker Solar Probe have some of these capabilities (see Sect. 13.3.1), but neither observes all the time and they both move with respect to the Sun-Earth line. Therefore they will not have the surveying capability of $\mathrm{SOHO}$.

To further our understanding of existing and future observations of near-Sun comets, comprehensive simulation capabilities need to be developed further in order to fully understand the complex interplay of numerous physical and chemical processes in the near-Sun environment (Sect. 8.4).

\subsection{Future Facilities}

Developments in ground-based observatories and space-based platforms will undoubtedly allow for the continued growth in the understanding of near-Sun comets.

\subsubsection{Space-Based Facilities}

ESA's Solar Orbiter mission, due to be launched in 2019, will make in situ and remote observations to within $0.284 \mathrm{AU}$, or $\sim 60 \mathrm{R}_{\odot}$ of the Sun. Its Multi Element Telescope for Imaging and Spectroscopy (METIS) instrument will produce simultaneous coronagraphic images in Ly $\alpha$ and white light. The length of the comet's Ly $\alpha$ tail corresponds to the ionisation time, and will therefore give an estimate of the coronal density. The opening angle 
of the Ly $\alpha$ tail corresponds to the ratio of the thermal speed of the hydrogen atoms to the relative speed of the comet and the solar wind, so it will give an estimate of the coronal proton temperature. The angle between the Ly $\alpha$ tail and the path of the comet corresponds to the ratio of the perpendicular component of the solar wind to the comet speed and, since the comet speed is known, it will provide an estimate of the solar wind speed. METIS will only observe for about 30 days per year, but during that time it should observe about a dozen sungrazing comets that will act as probes of the corona and inner solar wind (Bemporad et al. 2015).

NASA's Parker Solar Probe (Fox et al. 2016), due to launch in 2018, will approach to within $9.86 \mathrm{R}_{\odot}(0.0458 \mathrm{AU})$ of the Sun's centre, i.e. well within the perihelion distance range of sunskirters. Its instruments, some of which will carry out in situ measurements, include the Wide-Field Imager for Solar Probe Plus, WISPR, whose two telescopes will provide 36 " pixel $^{-1}$ views covering 2.2-20 $\mathrm{R}_{\odot}$ from the Sun at perihelion (Vourlidas et al. 2016).

Solar Orbiter and Parker Solar Probe will therefore provide in situ measurements of the environments encountered by near-Sun comets, may potentially provide in situ detections of comet signatures in the near-Sun environment, as well as high-resolution remote observations of near-Sun comets at relatively small distances.

To continue our monitoring of the near-Sun environment from Earth, missions that include coronagraphs do need to be planned to replace $\mathrm{SOHO}$ when it reaches the end of its operations. We note that the designs of coronagraphs and other solar instruments have naturally been driven by solar physics, but we suggest that capabilities to carry out multispectral observations of comets should be included in the design of future instruments.

NASA's James Webb Space Telescope (JWST), due to begin observing in 2019, will be uniquely capable of studying the small, potentially inactive nuclei of near-Sun comets at IR wavelengths. JWST's pointing and non-sidereal tracking constraints (see discussion in Kelley et al. 2016) will not allow observation of these comets when close to the Sun. However, its large $(6.5-\mathrm{m})$ mirror should allow studies of objects like 322P/SOHO 1 at heliocentric distances beyond $1 \mathrm{AU}$. With JWST, it should be possible to definitively determine such objects' nucleus size, and to conduct searches for $\mathrm{CO}, \mathrm{CO}_{2}$, and $\mathrm{H}_{2} \mathrm{O}$, the three most common volatiles in typical comets.

\subsubsection{Ground-Based Facilities}

The Daniel K. Inouye Solar Telescope, DKIST, is a $4 \mathrm{~m}$ telescope located in Maui, HI, USA, that will observe the Sun and its near-environment starting in 2018. DKIST lacks a coronagraph and is therefore not optimized for detecting near-Sun comets. However, its instrument suite-including adaptive optics-aided optical and infrared spectroscopy and polarimetrymay permit exciting new investigations of comets discovered sufficiently far in advance to plan specific observing sequences.

Finally, we note the anticipated growing capabilities for the potential detection of nearSun comets pre-perihelion, which will allow greater forward planning for the observation of any particularly interesting targets. Most notably, LSST is an $8.4 \mathrm{~m}$ telescope that is due to begin operations in Chile in 2019. Its deep sensitivity, wide field of view, and high cadence may allow the earlier detection of near-Sun comets, well before they reach the fields of view of space-based solar observatories. Furthermore, sophisticated software is under development for the detection of moving objects and the calculation of their orbits in close to real time, potentially allowing dedicated follow up with other facilities. 
Acknowledgements This work is a direct result of support by the International Space Science Institute, ISSI, Bern, Switzerland through the hosting and provision of financial support for an international team to discuss the science of near-Sun comets. The UK Science and Technology Facilities Council, STFC, is thanked for full support of KSB and YR, and partial support of GHJ and CS. A part of this work was performed at the Jet Propulsion Laboratory under a contract with NASA. AD acknowledges the support of the Belgian National Science Foundation, Fonds pour la formation à la Recherche dans l'Industrie et l'Agriculture, FRIA. JCB gratefully acknowledges the financial support of ISSI and of a UK Leverhulme Emeritus Fellowship and of visitor funds from the NASA Jet Propulsion Laboratory (R.W. Carlson). The work of JCR was supported by Grant HST-GO-12855 to the Smithsonian Astrophysical Observatory. MMK was supported by grants from NASA's Planetary Mission Data Analysis program and Outer Planet Research program. DCB greatly appreciates support from the US National Science Foundation, NSF, Planetary Astronomy Program under Grant No. 0908529 and the European Space Agency/European Space Technology Center Visiting Scientist Program. AF acknowledges support from STFC Consolidated Grant ST/L 000709/1. JKS gratefully acknowledges the support of his PhD advisor H. Jay Melosh. C. Opitom acknowledges the support of the Belgian Fonds de la Recherche Scientifique, FNRS. PW was supported by a contract with the NASA US Rosetta Project. CS received funding from the European Union Seventh Framework Programme (FP7/2007-2013) under grant agreement no. 268421. The authors are grateful to S. Prosser, Librarian at the Royal Astronomical Society, London, for valuable assistance.

Open Access This article is distributed under the terms of the Creative Commons Attribution 4.0 International License (http://creativecommons.org/licenses/by/4.0/), which permits unrestricted use, distribution, and reproduction in any medium, provided you give appropriate credit to the original author(s) and the source, provide a link to the Creative Commons license, and indicate if changes were made.

\section{References}

W.D.W. Abney, A. Schuster, On the total solar eclipse of May 17, 1882. Philos. Trans. R. Soc. Lond., Ser. I 175, 253-271 (1884)

M.F. A'Hearn, R.L. Millis, D.G. Schleicher, D.J. Osip, P.V. Birch, The ensemble properties of comets: results from narrowband photometry of 85 comets, 1976-1992. Icarus 118, 223-270 (1995). doi:10.1006/ icar.1995.1190

C. Alcock, C.C. Fristrom, R. Siegelman, On the number of comets around other single stars. Astrophys. J. 302, 462-476 (1986). doi:10.1086/164005

H. Alfvén, On the theory of comet tails. Tellus 9 (1957)

E. Asphaug, W. Benz, Size, density, and structure of comet Shoemaker-Levy 9 inferred from the physics of tidal breakup. Icarus 121, 225-248 (1996). doi:10.1006/icar.1996.0083

A.-T. Auger, M. Ramy El-Maarry, O. Groussin, L. Jorda, S. Bouley, A. Séjourné, C. Capanna, S. Höfner, H. Sierks, Meter-scale thermal contraction crack polygons on comet 67P/Churyumov-Gerasimenko, in AAS/Division for Planetary Sciences Meeting Abstracts. AAS/Division for Planetary Sciences Meeting Abstracts, vol. 47 (2015), p. 500

H.-U. Auster, I. Apathy, G. Berghofer, K.-H. Fornacon, A. Remizov, C. Carr, C. Güttler, G. Haerendel, P. Heinisch, D. Hercik, M. Hilchenbach, E. Kührt, W. Magnes, U. Motschmann, I. Richter, C.T. Russell, A. Przyklenk, K. Schwingenschuh, H. Sierks, K.-H. Glassmeier, The nonmagnetic nucleus of comet 67P/Churyumov-Gerasimenko. Science 349(1) (2015). doi:10.1126/science.aaa5102

M.E. Bailey, J.E. Chambers, G. Hahn, Origin of sungrazers-a frequent cometary end-state. Astron. Astrophys. 257, 315-322 (1992)

K. Battams, M.M. Knight, SOHO comets: 20-years and 3,000 objects later. ArXiv e-prints (2016)

K. Battams, G.V. Williams, Comet C/2011 N3 (SOHO). Int. Astron. U. Circ. 9227 (2011)

E. Bear, N. Soker, Transient outburst events from tidally disrupted asteroids near white dwarfs. New Astron. 19, 56-61 (2013). doi:10.1016/j.newast.2012.08.004

E. Bear, N. Soker, Planetary systems and real planetary nebulae from planet destruction near white dwarfs. Mon. Not. R. Astron. Soc. 450, 4233-4239 (2015). doi:10.1093/mnras/stv921

E.E. Becklin, J.A. Westphal, Infrared observations of comet 1965f. Astrophys. J. 145, 445 (1966). doi:10.1086/148785

A. Bemporad, G. Poletto, J.C. Raymond, D.A. Biesecker, B. Marsden, P. Lamy, Y.-K. Ko, M. Uzzo, UVCS observation of sungrazer $\mathrm{C} / 2001 \mathrm{C} 2$ : possible comet fragmentation and plasma-dust interactions. Astrophys. J. 620, 523-536 (2005). doi:10.1086/427063

A. Bemporad, G. Poletto, J. Raymond, S. Giordano, A review of SOHO/UVCS observations of sungrazing comets. Planet. Space Sci. 55, 1021-1030 (2007). doi:10.1016/j.pss.2006.11.013 
A. Bemporad, S. Giordano, J.C. Raymond, M.M. Knight, Study of sungrazing comets with space-based coronagraphs: new possibilities offered by METIS on board Solar Orbiter. Adv. Space Res. 56, 22882297 (2015). doi:10.1016/j.asr.2015.08.037

M. Benna, P. Mahaffy, Multi-fluid model of comet 1P/Halley. Planet. Space Sci. 55, 1031-1043 (2007). doi:10.1016/j.pss.2006.11.019

J.L. Bertaux, E. Kyrölä, E. Quémerais, R. Pellinen, R. Lallement, W. Schmidt, M. Berthé, E. Dimarellis, J.P. Goutail, C. Taulemesse, C. Bernard, G. Leppelmeier, T. Summanen, H. Hannula, H. Huomo, V. Kehlä, S. Korpela, K. Leppälä, E. Strömmer, J. Torsti, K. Viherkanto, J.F. Hochedez, G. Chretiennot, R. Peyroux, T. Holzer, SWAN: a study of solar wind anisotropies on SOHO with Lyman alpha sky mapping. Sol. Phys. 162, 403-439 (1995). doi:10.1007/BF00733435

J.-L. Bertaux, M.R. Combi, E. Quémerais, W. Schmidt, The water production rate of Rosetta target comet 67P/Churyumov-Gerasimenko near perihelion in 1996, 2002 and 2009 from Lyman $\alpha$ observations with SWAN/SOHO. Planet. Space Sci. 91, 14-19 (2014). doi:10.1016/j.pss.2013.11.006

D. Bewsher, D.S. Brown, C.J. Eyles, B.J. Kellett, G.J. White, B. Swinyard, Determination of the photometric calibration and large-scale flatfield of the STEREO heliospheric imagers: I. HI-1. Sol. Phys. 264, 433460 (2010). doi:10.1007/s11207-010-9582-8

V. Bezugly, M. Kusiak, R. Matson, K. Battams, G.V. Williams, Comet C/2012 E2 (Swan). Central Bureau Electronic Telegrams 3047 (2012)

D.A. Biesecker, P. Lamy, O.C. St. Cyr, A. Llebaria, R.A. Howard, Sungrazing comets discovered with the SOHO/LASCO coronagraphs 1996-1998. Icarus 157, 323-348 (2002). doi:10.1006/icar.2002.6827

K.S. Birkett, Investigating the neutral sodium emissions observed at comets, $\mathrm{PhD}$ thesis, UCL, University College London, Gower Street, London WC1E 6BT, UK (2017). http://discovery.ucl.ac.uk/1541019/

H. Boehnhardt, Split comets, in Comets II, ed. by M.C. Festou, H.U. Keller, H.A. Weaver (Univ. of Arizona Press/Lunar Planet. Inst., Tucson/Houston, 2004), pp. 301-316

D.C. Boice, R. Wegmann, The Deep Space 1 encounter with comet 19P/Borrelly. Adv. Space Res. 39, 407412 (2007). doi:10.1016/j.asr.2003.02.092

D.C. Boice, W.F. Huebner, J.J. Keady, H.U. Schmidt, R. Wegmann, A model of comet P/Giacobini-Zinner. Geophys. Res. Lett. 13, 381-384 (1986). doi:10.1029/GL013i004p00381

D.C. Boice, L.A. Soderblom, D.T. Britt, R.H. Brown, B.R. Sandel, R.V. Yelle, B.J. Buratti, M.D. Hicks, R.M. Nelson, M.D. Rayman, J. Oberst, N. Thomas, The Deep Space 1 encounter with comet 19p/Borrelly. Earth Moon Planets 89, 301-324 (2002). doi:10.1023/A:1021519124588

D.C. Boice, Suisei-a versatile global model of comets with applications to small solar system bodies. J. Appl. Math. Phys. 5(2), 311-320 (2017). doi:10.4236/jamp.2017.52028

B.P. Bonev, M.A. DiSanti, G.L. Villanueva, E.L. Gibb, L. Paganini, M.J. Mumma, The inner coma of comet C/2012 S1 (ISON) at $0.53 \mathrm{AU}$ and $0.35 \mathrm{AU}$ from the Sun. Astrophys. J. Lett. 796, 6 (2014). doi:10.1088/2041-8205/796/1/L6

J.E. Bortle, Post-perihelion survival of comets with small q. Int. Comet Q. 13, 89 (1991)

A.P. Boss, A.G.W. Cameron, W. Benz, Tidal disruption of inviscid planetesimals. Icarus 92, 165-178 (1991). doi:10.1016/0019-1035(91)90042-R

W.F. Bottke, A. Morbidelli, R. Jedicke, J.-M. Petit, H.F. Levison, P. Michel, T.S. Metcalfe, Debiased orbital and absolute magnitude distribution of the near-Earth objects. Icarus 156, 399-433 (2002). doi:10.1006/icar.2001.6788

J.C. Brandt, M. Snow, Heliospheric latitude variations of properties of cometary plasma tails: a test of the Ulysses comet watch paradigm. Icarus 148, 52-64 (2000). doi:10.1006/icar.2000.6484

J. Brown, R. Carlson, M. Toner, Destruction regimes of Sun-skimming and Sun-plunging comets, in Asteroids, Comets, Meteors 2014, ed. by K. Muinonen, A. Penttilä, M. Granvik, A. Virkki, G. Fedorets, O. Wilkman, T. Kohout (2014)

J.C. Brown, The deduction of energy spectra of non-thermal electrons in flares from the observed dynamic spectra of hard X-ray bursts. Sol. Phys. 18, 489-502 (1971). doi:10.1007/BF00149070

J.C. Brown, V.A. Carlaw, J.P. Cassinelli, Finite source depolarization factors for circumstellar scattering. Astrophys. J. 344, 341-349 (1989). doi:10.1086/167803

J.C. Brown, R.W. Carlson, M.P. Toner, Destruction and observational signatures of Sun-impacting comets. Astrophys. J. 807, 165 (2015). doi:10.1088/0004-637X/807/2/165

J.C. Brown, D. Veras, B.T. Gänsicke, Deposition of steeply infalling debris around white dwarf stars. Mon. Not. R. Astron. Soc. 468, 1575-1593 (2017). doi:10.1093/mnras/stx428

J.C. Brown, H.E. Potts, L.J. Porter, G. le Chat, Mass loss, destruction and detection of Sun-grazing and -impacting cometary nuclei. ArXiv e-prints (2011) 
G.E. Brueckner, R.A. Howard, M.J. Koomen, C.M. Korendyke, D.J. Michels, J.D. Moses, D.G. Socker, K.P. Dere, P.L. Lamy, A. Llebaria, M.V. Bout, R. Schwenn, G.M. Simnett, D.K. Bedford, C.J. Eyles, The Large Angle Spectroscopic Coronagraph (LASCO). Sol. Phys. 162, 357-402 (1995). doi:10.1007/ BF00733434

P. Bryans, W.D. Pesnell, The extreme-ultraviolet emission from Sun-grazing comets. Astrophys. J. 760, 18 (2012). doi:10.1088/0004-637X/760/1/18

P. Bryans, W.D. Pesnell, On the absence of EUV emission from comet C/2012 S1 (ISON). Astrophys. J. 822, 77 (2016). doi:10.3847/0004-637X/822/2/77

M. Bzowski, M. Królikowska, Are the sungrazing comets the inner source of pickup ions and energetic neutral atoms? Astron. Astrophys. 435, 723-732 (2005). doi:10.1051/0004-6361:20041169

W.W. Campbell, R. Trumpler, Search for intramercurial bodies. Publ. Astron. Soc. Pac. 35, 214 (1923). doi:10.1086/123310

H. Campins, M.S. Kelley, Y. Fernández, J. Licandro, K. Hargrove, Low perihelion near-Earth asteroids. Earth Moon Planets 105, 159-165 (2009). doi:10.1007/s11038-009-9310-2

J.P. Cassinelli, K.H. Nordsieck, M.A. Murison, Polarization of light scattered from the winds of early-type stars. Astrophys. J. 317, 290-302 (1987). doi:10.1086/165277

D. Chochol, V. Rusin, L. Kulcar, V. Vanysek, Emission features in the solar corona after the perihelion passage of comet 1979 XI. Astrophys. Space Sci. 91, 71-77 (1983). doi:10.1007/BF00650215

P.W. Chodas, D.K. Yeomans, The orbital motion and impact circumstances of comet Shoemaker-Levy 9, in IAU Colloq. 156: The Collision of Comet Shoemaker-Levy 9 and Jupiter, ed. by K.S. Noll, H.A. Weaver, P.D. Feldman (1996), pp. 1-30

C.F. Chyba, P.J. Thomas, K.J. Zahnle, The 1908 Tunguska explosion-atmospheric disruption of a stony asteroid. Nature 361, 40-44 (1993). doi:10.1038/361040a0

A. Ciaravella, J.C. Raymond, S. Giordano, Ultraviolet spectra of the C-2003K7 comet: evidence for dust sublimation in Si and C lines. Astrophys. J. Lett. 713, 69-73 (2010). doi:10.1088/2041-8205/713/1/L69

M.R. Combi, Z. Boyd, Y. Lee, T.S. Patel, J.-L. Bertaux, E. Quémerais, J.T.T. Mäkinen, SOHO/SWAN observations of comets with small perihelia: C/2002 V1 (NEAT), C/2002 X5 (Kudo-Fujikawa), 2006 P1 (McNaught) and 96P/Machholz 1. Icarus 216, 449-461 (2011). doi:10.1016/j.icarus.2011.09.019

M.R. Combi, N. Fougere, J.T.T. Mäkinen, J.-L. Bertaux, E. Quémerais, S. Ferron, Unusual water production activity of comet C/2012 S1 (ISON): outbursts and continuous fragmentation. Astrophys. J. Lett. 788, 7 (2014). doi:10.1088/2041-8205/788/1/L7

M.A. Cordiner, A.J. Remijan, J. Boissier, S.N. Milam, M.J. Mumma, S.B. Charnley, L. Paganini, G. Villanueva, D. Bockelée-Morvan, Y.-J. Kuan, Y.-L. Chuang, D.C. Lis, N. Biver, J. Crovisier, D. Minniti, I.M. Coulson, Mapping the release of volatiles in the inner comae of comets C/2012 F6 (Lemmon) and C/2012 S1 (ISON) using the Atacama Large Millimeter/Submillimeter Array. Astrophys. J. Lett. 792, 2 (2014). doi:10.1088/2041-8205/792/1/L2

J.J. Cowan, M.F. A'Hearn, Vaporization of comet nuclei-light curves and life times. Moon Planets 21, 155171 (1979). doi:10.1007/BF00897085

D.P. Cox, W.H. Tucker, Ionization equilibrium and radiative cooling of a low-density plasma. Astrophys. J. 157, 1157 (1969). doi:10.1086/150144

G. Cremonese, Hale-Bopp and its sodium tails. Space Sci. Rev. 90, 83-89 (1999). doi:10.1023/A: 1005281611036

T.E. Cravens, X-ray emission from comets. Science 296, 1042-1046 (2002). doi:10.1126/science.1070001

G. Cremonese, H. Boehnhardt, J. Crovisier, H. Rauer, A. Fitzsimmons, M. Fulle, J. Licandro, D. Pollacco, G.P. Tozzi, R.M. West, Neutral sodium from comet Hale-Bopp: a third type of tail. Astrophys. J. Lett. 490, 199-202 (1997). doi:10.1086/311040

G. Cremonese, W.F. Huebner, H. Rauer, D.C. Boice, Neutral sodium tails in comets. Adv. Space Res. 29, 1187-1197 (2002). doi:10.1016/S0273-1177(02)00136-9

W. Curdt, H. Boehnhardt, J.-B. Vincent, S.K. Solanki, U. Schühle, L. Teriaca, Scattered Lyman- $\alpha$ radiation of comet 2012/S1 (ISON) observed by SUMER/SOHO. Astron. Astrophys. 567, 1 (2014). doi:10.1051/0004-6361/201423990

G.W. Curtis, The Sacramento Peak Observatory Staff, Daylight observations of the 1965 F comet at the Sacramento Peak Observatory. Astron. J. 71, 194-196 (1966). doi:10.1086/109902

B.J.R. Davidsson, Tidal splitting and rotational breakup of solid spheres. Icarus 142, 525-535 (1999). doi:10.1006/icar.1999.6214

B.J.R. Davidsson, Tidal splitting and rotational breakup of solid biaxial ellipsoids. Icarus 149, 375-383 (2001). doi:10.1006/icar.2000.6540

B.J.R. Davidsson, H. Sierks, C. Güttler, F. Marzari, M. Pajola, H. Rickman, M.F. A'Hearn, A.-T. Auger, M.R. El-Maarry, S. Fornasier, P.J. Gutiérrez, H.U. Keller, M. Massironi, C. Snodgrass, J.-B. Vincent, C. Barbieri, P.L. Lamy, R. Rodrigo, D. Koschny, M.A. Barucci, J.-L. Bertaux, I. Bertini, G. Cremonese, V. Da Deppo, S. Debei, M. De Cecco, C. Feller, M. Fulle, O. Groussin, S.F. Hviid, S. Höfner, W.-H. 
Ip, L. Jorda, J. Knollenberg, G. Kovacs, J.-R. Kramm, E. Kührt, M. Küppers, F. La Forgia, L.M. Lara, M. Lazzarin, J.J. Lopez Moreno, R. Moissl-Fraund, S. Mottola, G. Naletto, N. Oklay, N. Thomas, C. Tubiana, The primordial nucleus of comet 67P/Churyumov-Gerasimenko. Astron. Astrophys. 592, 63 (2016). doi:10.1051/0004-6361/201526968

D. de Winter, C.A. Grady, M.E. van den Ancker, M.R. Pérez, C. Eiroa, Episodic accretion around the Herbig AE star BF Orionis. Evidence for the presence of extra-solar comets. Astron. Astrophys. 343, 137-150 (1999)

W.A. Delamere, A.S. McEwen, J.-Y. Li, C.M. Lisse, B.P. Bonev, M.A. DiSanti, E.L. Gibb, G.L. Villanueva, L. Paganini, M.J. Mumma, G.V. Williams, Comet C/2012 S1 (Ison). Central Bureau Electronic Telegrams 3720 (2013)

A.R. Dobrovolskis, Tidal disruption of solid bodies. Icarus 88, 24-38 (1990). doi:10.1016/00191035(90)90175-9

B. Donn, D. Hughes, A fractal model of a cometary nucleus formed by random accretion, in ESLAB Symposium on the Exploration of Halley's Comet, ed. by B. Battrick, E.J. Rolfe, R. Reinhard ESA Special Publication, vol. 250 (1986)

C. Downs, J.A. Linker, Z. Mikic, P. Riley, C.J. Schrijver, P. Saint-Hilaire, Probing the solar magnetic field with a Sun-grazing comet. Science 340, 1196-1199 (2013)

M. Drahus, Rotational disruption of comets with parabolic orbits, in AAS/Division for Planetary Sciences Meeting Abstracts. AAS/Division for Planetary Sciences Meeting Abstracts, vol. 46 (2014), p. 200

M. Druckmüller, S.R. Habbal, P. Aniol, A. Ding, H. Morgan, Imaging comet ISON C/2012 S1 in the inner corona at perihelion. Astrophys. J. Lett. 784, 22 (2014). doi:10.1088/2041-8205/784/2/L22

D.D. Durda, S.A. Stern, W.B. Colwell, J.W. Parker, H.F. Levison, D.M. Hassler, A new observational search for vulcanoids in SOHO/LASCO coronagraph images. Icarus 148, 312-315 (2000). doi:10.1006/icar.2000.6520

D. Eichler, D. Mordecai, Comet encounters and Carbon 14. Astrophys. J. Lett. 761, 27 (2012). doi:10.1088/ 2041-8205/761/2/L27

Y. Ellinger, F. Pauzat, O. Mousis, A. Guilbert-Lepoutre, F. Leblanc, M. Ali-Dib, M. Doronin, E. Zicler, A. Doressoundiram, Neutral $\mathrm{Na}$ in cometary tails as a remnant of early aqueous alteration. Astrophys. J. Lett. 801, 30 (2015). doi:10.1088/2041-8205/801/2/L30

A.G. Emslie, The collisional interaction of a beam of charged particles with a hydrogen target of arbitrary ionization level. Astrophys. J. 224, 241-246 (1978). doi:10.1086/156371

K.J. England, Early sungrazer comets. J. Br. Astron. Assoc. 112, 13-28 (2002)

C. Evans, J. McKim Malville, Sodium D lines in comet 1965 F near perihelion passage. Publ. Astron. Soc. Pac. 79, 310-316 (1967). doi:10.1086/128489

E. Everhart, Intrinsic distributions of cometary perihelia and magnitudes. Astron. J. 72, 1002-1011 (1967)

J. Farihi, B.T. Gänsicke, D. Koester, Evidence for water in the rocky debris of a disrupted extrasolar minor planet. Science 342, 218-220 (2013). doi:10.1126/science.1239447

P. Farinella, C. Froeschle, R. Gonczi, G. Hahn, A. Morbidelli, G.B. Valsecchi, Asteroids falling into the Sun. Nature 371, 314-317 (1994). doi:10.1038/371314a0

T.L. Farnham, D.G. Schleicher, M.F. A'Hearn, The HB narrowband comet filters: standard stars and calibrations. Icarus 147, 180-204 (2000). doi:10.1006/icar.2000.6420

T.L. Farnham, N.H. Samarasinha, B.E.A. Mueller, M.M. Knight, Cyanogen jets and the rotation state of comet Machholz (C/2004 Q2). Astron. J. 133, 2001-2007 (2007). doi:10.1086/513186

Y.R. Fernández, M.S. Kelley, P.L. Lamy, I. Toth, O. Groussin, C.M. Lisse, M.F. A'Hearn, J.M. Bauer, H. Campins, A. Fitzsimmons, J. Licandro, S.C. Lowry, K.J. Meech, J. Pittichová, W.T. Reach, C. Snodgrass, H.A. Weaver, Thermal properties, sizes, and size distribution of Jupiter-family cometary nuclei. Icarus 226, 1138-1170 (2013). doi:10.1016/j.icarus.2013.07.021

N.J. Fox, M.C. Velli, S.D. Bale, R. Decker, A. Driesman, R.A. Howard, J.C. Kasper, J. Kinnison, M. Kusterer, D. Lario, M.K. Lockwood, D.J. McComas, N.E. Raouafi, A. Szabo, The Solar Probe Plus Mission: humanity's first visit to our star. Space Sci. Rev. 204, 7-48 (2016). doi:10.1007/s11214-015-0211-6

H.-E. Froehlich, P. Notni, Radiation pressure-a stabilizing agent of dust clouds in comets? Astron. Nachr. 309, 147-155 (1988). doi:10.1002/asna.2113090211

H.-E. Froehlich, P. Notni, W. Thaenert, The striae in the dust tails of great comets: a formal comparison of the goodness of fit by various theories, and a new proposal, in Diversity and Similarity of Comets, ed. by E.J. Rolfe, B. Battrick ESA Special Publication, vol. 278 (1987)

M. Fulle, F. Leblanc, R.A. Harrison, C.J. Davis, C.J. Eyles, J.P. Halain, R.A. Howard, D. Bockelée-Morvan, G. Cremonese, T. Scarmato, Discovery of the atomic iron tail of comet MCNaught using the heliospheric imager on STEREO. Astrophys. J. Lett. 661, 93-96 (2007). doi:10.1086/518719

M. Fulle, F. Marzari, V. Della Corte, S. Fornasier, H. Sierks, A. Rotundi, C. Barbieri, P.L. Lamy, R. Rodrigo, D. Koschny, H. Rickman, H.U. Keller, J.J. López-Moreno, M. Accolla, J. Agarwal, M.F. A’Hearn, N. Altobelli, M.A. Barucci, J.-L. Bertaux, I. Bertini, D. Bodewits, E. Bussoletti, L. Colangeli, M. Cosi, G. 
Cremonese, J.-F. Crifo, V. Da Deppo, B. Davidsson, S. Debei, M. De Cecco, F. Esposito, M. Ferrari, F. Giovane, B. Gustafson, S.F. Green, O. Groussin, E. Grün, P. Gutierrez, C. Güttler, M.L. Herranz, S.F. Hviid, W. Ip, S.L. Ivanovski, J.M. Jerónimo, L. Jorda, J. Knollenberg, R. Kramm, E. Kührt, M. Küppers, L. Lara, M. Lazzarin, M.R. Leese, A.C. López-Jiménez, F. Lucarelli, E. Mazzotta Epifani, J.A.M. McDonnell, V. Mennella, A. Molina, R. Morales, F. Moreno, S. Mottola, G. Naletto, N. Oklay, J.L. Ortiz, E. Palomba, P. Palumbo, J.-M. Perrin, F.J.M. Rietmeijer, J. Rodríguez, R. Sordini, N. Thomas, C. Tubiana, J.-B. Vincent, P. Weissman, K.-P. Wenzel, V. Zakharov, J.C. Zarnecki, Evolution of the dust size distribution of comet 67P/Churyumov-Gerasimenko from 2.2 AU to perihelion. Astrophys. J. 821, 19 (2016). doi:10.3847/0004-637X/821/1/19

B.T. Gänsicke, A. Aungwerojwit, T.R. Marsh, V.S. Dhillon, D.I. Sahman, D. Veras, J. Farihi, P. Chote, R. Ashley, S. Arjyotha, S. Rattanasoon, S.P. Littlefair, D. Pollacco, M.R. Burleigh, High-speed photometry of the disintegrating planetesimals at WD1145+017: evidence for rapid dynamical evolution. Astrophys. J. Lett. 818, 7 (2016). doi:10.3847/2041-8205/818/1/L7

S. Giordano, J.C. Raymond, P. Lamy, M. Uzzo, D. Dobrzycka, Probing the solar wind acceleration region with the Sun-grazing comet C/2002 S2. Astrophys. J. 798, 47 (2015). doi:10.1088/0004-637X/798/1/47

B.J. Gladman, F. Migliorini, A. Morbidelli, V. Zappala, P. Michel, A. Cellino, C. Froeschle, H.F. Levison, M. Bailey, M. Duncan, Dynamical lifetimes of objects injected into asteroid belt resonances. Science 277, 197-201 (1997). doi:10.1126/science.277.5323.197

G. Gloeckler, J. Geiss, N.A. Schwadron, L.A. Fisk, T.H. Zurbuchen, F.M. Ipavich, R. von Steiger, H. Balsiger, B. Wilken, Interception of comet Hyakutake's ion tail at a distance of 500 million kilometres. Nature 404, 576-578 (2000)

C. Goetz, C. Koenders, I. Richter, K. Altwegg, J. Burch, C. Carr, E. Cupido, A. Eriksson, C. Güttler, P. Henri, P. Mokashi, Z. Nemeth, H. Nilsson, M. Rubin, H. Sierks, B. Tsurutani, C. Vallat, M. Volwerk, K.-H. Glassmeier, First detection of a diamagnetic cavity at comet 67P/Churyumov-Gerasimenko. Astron. Astrophys. 588, 24 (2016). doi:10.1051/0004-6361/201527728

L. Golub, E. Deluca, G. Austin, J. Bookbinder, D. Caldwell, P. Cheimets, J. Cirtain, M. Cosmo, P. Reid, A. Sette, M. Weber, T. Sakao, R. Kano, K. Shibasaki, H. Hara, S. Tsuneta, K. Kumagai, T. Tamura, M. Shimojo, J. McCracken, J. Carpenter, H. Haight, R. Siler, E. Wright, J. Tucker, H. Rutledge, M. Barbera, G. Peres, S. Varisco, The X-Ray Telescope (XRT) for the Hinode Mission. Sol. Phys. 243, 63-86 (2007). doi:10.1007/s11207-007-0182-1

T.I. Gombosi, Charge exchange avalanche at the cometopause. Geophys. Res. Lett. 14, 1174-1177 (1987). doi:10.1029/GL014i011p01174

R. Gomes, H.F. Levison, K. Tsiganis, A. Morbidelli, Origin of the cataclysmic Late Heavy Bombardment period of the terrestrial planets. Nature 435, 466-469 (2005). doi:10.1038/nature03676

M. Granvik, A. Morbidelli, R. Jedicke, B. Bolin, W.F. Bottke, E. Beshore, D. Vokrouhlický, M. Delbò, P. Michel, Super-catastrophic disruption of asteroids at small perihelion distances. Nature 530, 303-306 (2016). doi:10.1038/nature16934

D.W.E. Green, H. Rickman, A.C. Porter, K.J. Meech, The strange periodic comet Machholz. Science 247, 1063-1067 (1990)

S. Green, C. Kowal, 1983 TB. Int. Astron. U. Circ. 3878 (1983)

S. Greenstreet, H. Ngo, B. Gladman, The orbital distribution of near-Earth objects inside Earth's orbit. Icarus 217, 355-366 (2012). doi:10.1016/j.icarus.2011.11.010

E. Grün, J. Agarwal, N. Altobelli, K. Altwegg, M.S. Bentley, N. Biver, V. Della Corte, N. Edberg, P.D. Feldman, M. Galand, B. Geiger, C. Götz, B. Grieger, C. Güttler, P. Henri, M. Hofstadter, M. Horanyi, E. Jehin, H. Krüger, S. Lee, T. Mannel, E. Morales, O. Mousis, M. Müller, C. Opitom, A. Rotundi, R. Schmied, F. Schmidt, H. Sierks, C. Snodgrass, R.H. Soja, M. Sommer, R. Srama, C.-Y. Tzou, J.-B. Vincent, P. Yanamandra-Fisher, M.F. A'Hearn, A.I. Erikson, C. Barbieri, M.A. Barucci, J.-L. Bertaux, I. Bertini, J. Burch, L. Colangeli, G. Cremonese, V. Da Deppo, B. Davidsson, S. Debei, M. De Cecco, J. Deller, L.M. Feaga, M. Ferrari, S. Fornasier, M. Fulle, A. Gicquel, M. Gillon, S.F. Green, O. Groussin, P.J. Gutiérrez, M. Hofmann, S.F. Hviid, W.-H. Ip, S. Ivanovski, L. Jorda, H.U. Keller, M.M. Knight, J. Knollenberg, D. Koschny, J.-R. Kramm, E. Kührt, M. Küppers, P.L. Lamy, L.M. Lara, M. Lazzarin, J.J. Lòpez-Moreno, J. Manfroid, E.M. Epifani, F. Marzari, G. Naletto, N. Oklay, P. Palumbo, J.W. Parker, H. Rickman, R. Rodrigo, J. Rodrìguez, E. Schindhelm, X. Shi, R. Sordini, A.J. Steffl, S.A. Stern, N. Thomas, C. Tubiana, H.A. Weaver, P. Weissman, V.V. Zakharov, M.G.G.T. Taylor, The 2016 Feb. 19 outburst of comet 67P/CG: an ESA Rosetta multi-instrument study. Mon. Not. R. Astron. Soc. 462, 220-234 (2016). doi:10.1093/mnras/stw2088

Y. Grynko, K. Jockers, R. Schwenn, The phase curve of cometary dust: observations of comet 96P/Machholz 1 at large phase angle with the SOHO LASCO C3 Coronagraph. Astron. Astrophys. 427, 755-761 (2004). doi:10.1051/0004-6361:20047131

B. Gundlach, J. Blum, Y.V. Skorov, H.U. Keller, A note on the survival of the sungrazing comet C/2011 W3 (Lovejoy) within the Roche limit. ArXiv e-prints (2012) 
D. Hammer, R. Kracht, R. Matson, D. Evans, J. Sachs, X. Leprette, S. Hoenig, B.G. Marsden, Comets C/2002 R4, R5, R6, R7, R8, S3, S4, S5 (SOHO). Int. Astron. U. Circ. 7984 (2002)

T. Heidarzadeh, A History of Physical Theories of Comets, from Aristotle to Whipple (Springer, Berlin, 2008)

J.R. Hill, D.A. Mendis, On the origin of striae in cometary dust tails. Astrophys. J. 242, 395-401 (1980). doi:10.1086/158472

J.R. Hill, D.A. Mendis, Electrostatic disruption of a charged conducting spheroid. Can. J. Phys. 59, 897-901 (1981). doi:10.1139/p81-116

D.C. Hines, G. Videen, E. Zubko, K. Muinonen, Y. Shkuratov, V.G. Kaydash, M.M. Knight, M.L. Sitko, C.M. Lisse, M. Mutchler, D. Hammer, P.A. Yanamandra-Fisher, Hubble Space Telescope pre-perihelion ACS/WFC imaging polarimetry of comet Ison (c/2012 s1) at 3.81 AU. Astrophys. J. Lett. 780, 32 (2014). doi:10.1088/2041-8205/780/2/L32

M. Hirabayashi, D.J. Scheeres, S.R. Chesley, S. Marchi, J.W. McMahon, J. Steckloff, S. Mottola, S.P. Naidu, T. Bowling, Fission and reconfiguration of bilobate comets as revealed by 67P/ChuryumovGerasimenko. Nature 534, 352-355 (2016). doi:10.1038/nature17670

T. Hoffman, B. Zhou, K. Battams, B.G. Marsden, Comets C/2007 M4, 2007 M5, 2007 M6 (SOHO). Minor Planet Electronic Circulars 2007-U15 (2007)

K.A. Holsapple, P. Michel, Tidal disruptions: a continuum theory for solid bodies. Icarus 183, 331-348 (2006). doi:10.1016/j.icarus.2006.03.013

S.F. Hönig, Identification of a new short-period comet near the Sun. Astron. Astrophys. 445, 759-763 (2006). doi:10.1051/0004-6361:20053991

R.A. Howard, J.D. Moses, A. Vourlidas, J.S. Newmark, D.G. Socker, S.P. Plunkett, C.M. Korendyke, J.W. Cook, A. Hurley, J.M. Davila, W.T. Thompson, O.C. St. Cyr, E. Mentzell, K. Mehalick, J.R. Lemen, J.P. Wuelser, D.W. Duncan, T.D. Tarbell, C.J. Wolfson, A. Moore, R.A. Harrison, N.R. Waltham, J. Lang, C.J. Davis, C.J. Eyles, H. Mapson-Menard, G.M. Simnett, J.P. Halain, J.M. Defise, E. Mazy, P. Rochus, R. Mercier, M.F. Ravet, F. Delmotte, F. Auchere, J.P. Delaboudiniere, V. Bothmer, W. Deutsch, D. Wang, N. Rich, S. Cooper, V. Stephens, G. Maahs, R. Baugh, D. McMullin, T. Carter, Sun Earth Connection Coronal and Heliospheric Investigation (SECCHI). Space Sci. Rev. 136, 67-115 (2008). doi:10.1007/s11214-008-9341-4

R. Howard, N. Koomen, D.J. Michels, N. Sheeley, B.G. Marsden, Probable sungrazing comet. Int. Astron. U. Circ. 3640, 1 (1981)

W.F. Huebner, Dust from cometary nuclei. Astron. Astrophys. 5, 286-297 (1970)

W.F. Huebner, D.C. Boice, N.A. Schwadron, Sungrazing comets as solar probes and dust analyzers. Adv. Space Res. 39, 413-420 (2007). doi:10.1016/j.asr.2006.09.042

W.F. Huebner, J. Benkhoff, M.-T. Capria, A. Coradini, C. De Sanctis, R. Orosei, D. Prialnik, Heat and Gas Diffusion in Comet Nuclei (ESA Publications Division, Noordwijk, 2006). Published for The International Space Science Institute, Bern, Switzerland

D.W. Hughes, The magnitude distribution, perihelion distribution and flux of long-period comets. Mon. Not. R. Astron. Soc. 326, 515-523 (2001). doi:10.1046/j.1365-8711.2001.04544.X

M.-T. Hui, Observations of comet P/2003 T12 = 2012 A3 (SOHO) at large phase angle in STEREO-B. Mon. Not. R. Astron. Soc. 436, 1564-1575 (2013). doi:10.1093/mnras/stt1683

M.-T. Hui, J. Li, Resurrection of (3200) Phaethon in 2016. Astron. J. 153, 23 (2017). doi:10.3847/ $1538-3881 / 153 / 1 / 23$

M.-T. Hui, Q.-Z. Ye, M. Knight, K. Battams, D. Clark, Gone in a blaze of glory: the demise of comet C/2015 D1 (SOHO). Astrophys. J. 813, 73 (2015). doi:10.1088/0004-637X/813/1/73

W.-H. Ip, A preliminary consideration of the electron impact ionization effect in cometary comas. Adv. Space Res. 5, 47-51 (1985). doi:10.1016/0273-1177(85)90066-3

M. Iseli, M. Küppers, W. Benz, P. Bochsler, Sungrazing comets: properties of nuclei and in situ detectability of cometary ions at 1 AU. Icarus 155, 350-364 (2002). doi:10.1006/icar.2001.6722

P.A. Isenberg, M.A. Lee, A dispersive analysis of bispherical pickup ion distributions. J. Geophys. Res. 101, 11055-11066 (1996). doi:10.1029/96JA00293

B.V. Jackson, A. Buffington, P.P. Hick, R.C. Altrock, S. Figueroa, P.E. Holladay, J.C. Johnston, S.W. Kahler, J.B. Mozer, S. Price, R.R. Radick, R. Sagalyn, D. Sinclair, G.M. Simnett, C.J. Eyles, M.P. Cooke, S.J. Tappin, T. Kuchar, D. Mizuno, D.F. Webb, P.A. Anderson, S.L. Keil, R.E. Gold, N.R. Waltham, The Solar Mass-Ejection Imager (SMEI) mission. Sol. Phys. 225, 177-207 (2004). doi:10.1007/s11207-004-2766-3

D. Jewitt, The active asteroids. Astrophys. J. 143, 66 (2012). doi:10.1088/0004-6256/143/3/66

D. Jewitt, Properties of near-Sun asteroids. Astron. J. 145, 133 (2013). doi:10.1088/0004-6256/145/5/133

D. Jewitt, J. Li, Activity in geminid parent (3200) Phaethon. Astron. J. 140, 1519-1527 (2010). doi:10.1088/0004-6256/140/5/1519

D. Jewitt, H. Hsieh, J. Agarwal, in The Active Asteroids, ed. by P. Michel, F.E. DeMeo, W.F. Bottke (2015), pp. 221-241.doi:10.2458/azu_uapress_9780816532131-ch012 
D. Jewitt, M. Mutchler, H. Weaver, M.-T. Hui, J. Agarwal, M. Ishiguro, J. Kleyna, J. Li, K. Meech, M. Micheli, R. Wainscoat, R. Weryk, Fragmentation kinematics in comet 332P/Ikeya-Murakami. Astrophys. J. Lett. 829, 8 (2016). doi:10.3847/2041-8205/829/1/L8

Y.D. Jia, C.T. Russell, L.K. Jian, W.B. Manchester, O. Cohen, A. Vourlidas, K.C. Hansen, M.R. Combi, T.I. Gombosi, Study of the 2007 April 20 CME-comet interaction event with an MHD model. Astrophys. J. 696, 56-60 (2009). doi:10.1088/0004-637X/696/1/L56

Y.D. Jia, Y.J. Ma, C.T. Russell, L.K. Jian, M.R. Combi, T.I. Gombosi, The physics of the interaction of a cometary dust tail with the solar wind, in EPSC-DPS Joint Meeting 2011 (2011), p. 98

Y.-D. Jia, C.T. Russell, W. Liu, Y.S. Shou, Multi-fluid model of a Sun-grazing comet in the rapidly ionizing, magnetized low corona. Astrophys. J. 796, 42 (2014). doi:10.1088/0004-637X/796/1/42

K. Jockers, The ion tail of comet Kohoutek 1973 XII during 17 days of solar wind gusts. Astron. Astrophys. Suppl. Ser. 62, 791-838 (1985)

G. Jones, K. Battams, Dust tail striae: lessons from recent comets, in Asteroids, Comets, Meteors 2014, ed. by K. Muinonen, A. Penttilä, M. Granvik, A. Virkki, G. Fedorets, O. Wilkman, T. Kohout (2014)

G.H. Jones, J.C. Brandt, The interaction of comet 153P/Ikeya-Zhang with interplanetary coronal mass ejections: identification of fast ICME signatures. Geophys. Res. Lett. 31, 20805 (2004). doi:10.1029/2004GL021166

G.H. Jones, A. Balogh, T.S. Horbury, Identification of comet Hyakutake's extremely long ion tail from magnetic field signatures. Nature 404, 574-576 (2000)

L. Jorda, R. Gaskell, C. Capanna, S. Hviid, P. Lamy, J. Durech, G. Faury, O. Groussin, P. Gutiérrez, C. Jackman, S.J. Keihm, H.U. Keller, J. Knollenberg, E. Kührt, S. Marchi, S. Mottola, E. Palmer, F.P. Schloerb, H. Sierks, J.-B. Vincent, M.F. A'Hearn, C. Barbieri, R. Rodrigo, D. Koschny, H. Rickman, M.A. Barucci, J.L. Bertaux, I. Bertini, G. Cremonese, V. Da Deppo, B. Davidsson, S. Debei, M. De Cecco, S. Fornasier, M. Fulle, C. Güttler, W.-H. Ip, J.R. Kramm, M. Küppers, L.M. Lara, M. Lazzarin, J.J. Lopez Moreno, F. Marzari, G. Naletto, N. Oklay, N. Thomas, C. Tubiana, K.-P. Wenzel, The global shape, density and rotation of comet 67P/Churyumov-Gerasimenko from preperihelion Rosetta/OSIRIS observations. Icarus 277, 257-278 (2016). doi:10.1016/j.icarus.2016.05.002

J.V. Keane, S.N. Milam, I.M. Coulson, J.T. Kleyna, Z. Sekanina, R. Kracht, T.-E. Riesen, K.J. Meech, S.B. Charnley, Catastrophic disruption of comet ISON. Astrophys. J. 831, 207 (2016). doi:10.3847/ 0004-637X/831/2/207

M.S.P. Kelley, C.E. Woodward, D. Bodewits, T.L. Farnham, M.S. Gudipati, D.E. Harker, D.C. Hines, M.M. Knight, L. Kolokolova, A. Li, I. de Pater, S. Protopapa, R.W. Russell, M.L. Sitko, D.H. Wooden, Cometary science with the James Webb Space Telescope. Publ. Astron. Soc. Pac. 128(1), 018009 (2016). doi:10.1088/1538-3873/128/959/018009

S.V. Kharchuk, P.P. Korsun, Striated features in the dust tail of comet C/2006 P1 (McNaught). Kinemat. Phys. Celest. Bodies 26, 322-325 (2010). doi:10.3103/S0884591310060048

F. Kiefer, A. Lecavelier des Etangs, J.-C. Augereau, A. Vidal-Madjar, A.-M. Lagrange, H. Beust, Exocomets in the circumstellar gas disk of HD 172555. Astron. Astrophys. 561, 10 (2014). doi:10.1051/ 0004-6361/201323128

H. Kimura, I. Mann, D.A. Biesecker, E.K. Jessberger, Dust grains in the comae and tails of sungrazing comets: modeling of their mineralogical and morphological properties. Icarus 159, 529-541 (2002). doi:10.1006/icar.2002.6940

D. Kirkwood, On the great southern comet of 1880. The Observatory 3, 590-592 (1880)

M. Knight, K. Battams, P/2007 R5 = 1999 R1 = 2003 R5 (SOHO). Int. Astron. U. Circ. 8872 (2007)

M.M. Knight, Studies of SOHO comets, PhD thesis, University of Maryland, College Park (2008)

M.M. Knight, K. Battams, Preliminary analysis of SOHO/STEREO observations of sungrazing comet ISON (C/2012 S1) around perihelion. Astrophys. J. Lett. 782, 37 (2014). doi:10.1088/2041-8205/782/2/L37

M.M. Knight, D.G. Schleicher, Observations of comet ISON (C/2012 S1) from Lowell Observatory. Astron. J. 149, 19 (2015). doi:10.1088/0004-6256/149/1/19

M.M. Knight, K.J. Walsh, Will comet ISON (C/2012 S1) survive perihelion? Astrophys. J. Lett. 776, 5 (2013). doi:10.1088/2041-8205/776/1/L5

M.M. Knight, M.F. A’Hearn, D.A. Biesecker, G. Faury, D.P. Hamilton, P. Lamy, A. Llebaria, Photometric study of the Kreutz comets observed by SOHO from 1996 to 2005. Astron. J. 139, 926-949 (2010). doi:10.1088/0004-6256/139/3/926

M.M. Knight, M.S. Kelley, H.A. Weaver, Y.R. Fernandez, S.R. Chesley, R. McNaught, D. Bodewits, C.M. Lisse, D.J. Osip, N. Dello Russo, K. Battams, A multiwavelength investigation of sungrazing comet Lovejoy (C/2011 W3), in AAS/Division for Planetary Sciences Meeting Abstracts, vol. 44 (2012), p. 514

M.M. Knight, A. Fitzsimmons, M.S.P. Kelley, C. Snodgrass, Comet 322P/SOHO 1: an asteroid with the smallest perihelion distance? Astrophys. J. Lett. 823, 6 (2016). doi:10.3847/2041-8205/823/1/L6

J.L. Kohl, R. Esser, L.D. Gardner, S. Habbal, P.S. Daigneau, E.F. Dennis, G.U. Nystrom, A. Panasyuk, J.C. Raymond, P.L. Smith, L. Strachan, A.A. van Ballegooijen, G. Noci, S. Fineschi, M. Romoli, A. Ciar- 
avella, A. Modigliani, M.C.E. Huber, E. Antonucci, C. Benna, S. Giordano, G. Tondello, P. Nicolosi, G. Naletto, C. Pernechele, D. Spadaro, G. Poletto, S. Livi, O. von der Lühe, J. Geiss, J.G. Timothy, G. Gloeckler, A. Allegra, G. Basile, R. Brusa, B. Wood, O.H.W. Siegmund, W. Fowler, R. Fisher, M. Jhabvala, The ultraviolet coronagraph spectrometer for the solar and heliospheric observatory. Sol. Phys. 162, 313-356 (1995). doi:10.1007/BF00733433

J.L. Kohl, G. Noci, S.R. Cranmer, J.C. Raymond, Ultraviolet spectroscopy of the extended solar corona. Astron. Astrophys. Rev. 13, 31-157 (2006). doi:10.1007/s00159-005-0026-7

R. Kokotanekova, C. Snodgrass, P. Lacerda, S.F. Green, S.C. Lowry, Y.R. Fernández, C. Tubiana, A. Fitzsimmons, H.H. Hsieh, Rotation of cometary nuclei: new light curves and an update of the ensemble properties of Jupiter-family comets. Mon. Not. R. Astron. Soc. 471, 2974-3007 (2017). doi:10.1093/mnras/stx 1716

L. Kolokolova, M.S. Hanner, A.-C. Levasseur-Regourd, B.Å.S. Gustafson, Physical properties of cometary dust from light scattering and thermal emission, in Comets II, ed. by M.C. Festou, H.U. Keller, H.A. Weaver (Univ. of Arizona Press/Lunar Planet. Inst., Tucson/Houston, 2004), pp. 577-604

I. Konno, W.F. Huebner, D.C. Boice, A model of dust fragmentation in near-nucleus jet-like features on comet P/Halley. Icarus 101, 84-94 (1993). doi:10.1006/icar.1993.1008

M.J. Koomen, J.D. Purcell, R. Tousey, Rocket observations of the corona on March 7, 1970. Nature 226, 1135-1138 (1970). doi:10.1038/2261135a0

Y. Kozai, Secular perturbations of asteroids with high inclination and eccentricity. Astron. J. 67, 591 (1962). doi: $10.1086 / 108790$

R. Kracht, B.G. Marsden, Comet C/1981 W1 (Solwind). Int. Astron. U. Circ. 8566 (2005a)

R. Kracht, B.G. Marsden, Comet C/1984 R1 (Solwind). Int. Astron. U. Circ. 8583 (2005b)

R. Kracht, B.G. Marsden, Comets C/1983 N2 and C/1984 Q1 (Solwind). Int. Astron. U. Circ. 8573 (2005c)

R. Kracht, B.G. Marsden, Comet C/1999 X3 = 2004 E2 = 2008 K10 (SOHO). Minor Planet Electronic Circulars 2008 (2008)

R. Kracht, M. Meyer, D. Hammer, B.G. Marsden, D.A.J. Seargent, COMETS C/1998 A2, 1998 A3, 2000 B8 (SOHO). Minor Planet Electronic Circulars 2002 (2002a)

R. Kracht, S. Hoenig, D. Hammer, B.G. Marsden, Comets C/1999 M3, 2002 E1 (SOHO). Minor Planet Electronic Circulars, 18 (2002b)

R. Kracht, B.G. Marsden, K. Battams, Z. Sekanina, Comets C/2008 L5-L8 (SOHO). Int. Astron. U. Circ. 8983 (2008)

E.A. Kramer, Y.R. Fernandez, C.M. Lisse, M.S.P. Kelley, L.M. Woodney, A dynamical analysis of the dust tail of comet C/1995 O1 (Hale-Bopp) at high heliocentric distances. Icarus 236, 136-145 (2014). doi:10.1016/j.icarus.2014.03.033

L. Kresák, A meteor mission into the orbit of Sun-grazing comets. Bull. Astron. Inst. Czechoslov. 17, 188195 (1966)

H. Kreutz, Untersuchungen Über das Cometensystem 1843 I, 1880 I und 1882 II (Druck von C. Schaidt, C. F. Mohr nachfl., Kiel, 1888), p. 92

H. Kreutz, Untersuchungen Über das System der Cometen 1843 I, 1880 I und 1882 II Theil II. Publication der Koeniglichen Sternwarte in Kiel 6 (1891)

H. Kreutz, Anzeige betr. Ergänzungshefte zu den Astr. Nachrichten. Astron. Nachr. 155, 63 (1901)

G.W. Kronk, Cometography: A Catalog of Comets. Volume 1, Ancient-1799 (Cambridge University Press, Cambridge/New York, 1999)

G.W. Kronk, Cometography: A Catalog of Comets. Volume 2, 1800-1899 (Cambridge University Press, Cambridge/New York, 2003)

G.W. Kronk, Cometography: A Catalog of Comets. Volume 3, 1900-1932 (Cambridge University Press, Cambridge/New York, 2007)

G.W. Kronk, M. Meyer, Cometography: A Catalog of Comets. Volume 5, 1960-1982 (Cambridge University Press, Cambridge/New York, 2010)

T.A. Kuchar, A. Buffington, C.N. Arge, P.P. Hick, T.A. Howard, B.V. Jackson, J.C. Johnston, D.R. Mizuno, S.J. Tappin, D.F. Webb, Observations of a comet tail disruption induced by the passage of a CME. J. Geophys. Res. Space Phys. 113, 04101 (2008). doi:10.1029/2007JA012603

R. Lallement, J.-L. Bertaux, K. Szegö, S. Nemeth, The shadow of comet Hale-Bopp in Lyman-Alpha. Earth Moon Planets 90, 67-76 (2002)

H. Lamy, V. Pierrard, M. Maksimovic, J.F. Lemaire, A kinetic exospheric model of the solar wind with a nonmonotonic potential energy for the protons. J. Geophys. Res. Space Phys. 108, 1047 (2003a). doi:10.1029/2002JA009487

P. Lamy, D.A. Biesecker, O. Groussin, SOHO/LASCO observation of an outburst of comet 2P/Encke at its 2000 perihelion passage. Icarus 163, 142-149 (2003b). doi:10.1016/S0019-1035(03)00040-X

P.L. Lamy, I. Toth, H.A. Weaver, Hubble Space Telescope observations of the nucleus of comet C/2012 S1 (ISON). Astrophys. J. Lett. 794, 9 (2014). doi:10.1088/2041-8205/794/1/L9 
P.L. Lamy, I. Toth, Y.R. Fernandez, H.A. Weaver, The sizes, shapes, albedos, and colors of cometary nuclei, in Comets II, ed. by M.C. Festou, H.U. Keller, H.A. Weaver (Univ. of Arizona Press/Lunar Planet. Inst., Tucson/Houston, 2004), pp. 223-264

P. Lamy, G. Faury, A. Llebaria, M.M. Knight, M.F. A'Hearn, K. Battams, Sunskirting comets discovered with the LASCO coronagraphs over the decade 1996-2008. Icarus 226, 1350-1398 (2013). doi:10.1016/j.icarus.2013.07.035

U.J. Le Verrier, Theorie du mouvement de Mercure. Annales de l'Observatoire de Paris 5 (1859)

M.A. Leake, C.R. Chapman, S.J. Weidenschilling, D.R. Davis, R. Greenberg, The chronology of Mercury's geological and geophysical evolution-the vulcanoid hypothesis. Icarus 71, 350-375 (1987). doi:10.1016/0019-1035(87)90034-0

F. Leblanc, M. Fulle, A. López Ariste, G. Cremonese, A. Doressoundiram, A. Sainz Dalda, B. Gelly, Comet McNaught C/2006 P1: observation of the sodium emission by the solar telescope THEMIS. Astron. Astrophys. 482, 293-298 (2008). doi:10.1051/0004-6361:20078795

J.R. Lemen, A.M. Title, D.J. Akin, P.F. Boerner, C. Chou, J.F. Drake, D.W. Duncan, C.G. Edwards, F.M. Friedlaender, G.F. Heyman, N.E. Hurlburt, N.L. Katz, G.D. Kushner, M. Levay, R.W. Lindgren, D.P. Mathur, E.L. McFeaters, S. Mitchell, R.A. Rehse, C.J. Schrijver, L.A. Springer, R.A. Stern, T.D. Tarbell, J.-P. Wuelser, C.J. Wolfson, C. Yanari, J.A. Bookbinder, P.N. Cheimets, D. Caldwell, E.E. Deluca, R. Gates, L. Golub, S. Park, W.A. Podgorski, R.I. Bush, P.H. Scherrer, M.A. Gummin, P. Smith, G. Auker, P. Jerram, P. Pool, R. Soufli, D.L. Windt, S. Beardsley, M. Clapp, J. Lang, N. Waltham, The Atmospheric Imaging Assembly (AIA) on the Solar Dynamics Observatory (SDO). Sol. Phys. 275, 17-40 (2012). doi:10.1007/s11207-011-9776-8

H.F. Levison, Comet taxonomy, in Completing the Inventory of the Solar System, ed. by T. Rettig, J.M. Hahn Astronomical Society of the Pacific Conference Series, vol. 107 (ASP, San Francisco, 1996), pp. 173191

H.F. Levison, M.J. Duncan, From the Kuiper belt to Jupiter-family comets: the spatial distribution of ecliptic comets. Icarus 127, 13-32 (1997). doi:10.1006/icar.1996.5637

J. Li, D. Jewitt, Recurrent perihelion activity in (3200) Phaethon. Astron. J. 145, 9 (2013). doi: 10.1088/0004-6256/145/6/154

J.-Y. Li, M.S.P. Kelley, M.M. Knight, T.L. Farnham, H.A. Weaver, M.F. A'Hearn, M.J. Mutchler, L. Kolokolova, P. Lamy, I. Toth, Characterizing the dust coma of comet C/2012 S1 (ISON) at $4.15 \mathrm{AU}$ from the Sun. Astrophys. J. Lett. 779, 3-5 (2013). doi:10.1088/2041-8205/779/1/L3

M.L. Lidov, The evolution of orbits of artificial satellites of planets under the action of gravitational perturbations of external bodies. Planet. Space Sci. 9, 719-759 (1962). doi:10.1016/0032-0633(62)90129-0

C. Lindsey, A.-C. Donea, Mechanics of seismic emission from solar flares. Sol. Phys. 251, 627-639 (2008). doi:10.1007/s11207-008-9140-9

C.M. Lisse, T.E. Cravens, K. Dennerl, X-ray and extreme ultraviolet emission from comets, in Comets II, ed. by M.C. Festou, H.U. Keller, H.A. Weaver (2004), pp. 631-643

T. Lovejoy, G.V. Williams, Comet C/2011 W3 (Lovejoy), in Central Bureau Electronic Telegrams, vol. 2930, (2011), p. 1

M.-M. Mac Low, K. Zahnle, Explosion of comet Shoemaker-Levy 9 on entry into the Jovian atmosphere. Astrophys. J. Lett. 434, 33-36 (1994). doi:10.1086/187565

R.M. MacQueen, O.C. St. Cyr, Sungrazing comets observed by the solar maximum mission coronagraph. Icarus 90, 96-106 (1991). doi:10.1016/0019-1035(91)90071-Z

R.M. MacQueen, J.A. Eddy, J.T. Gosling, E. Hildner, R.H. Munro, G.A. Newkirk Jr., A.I. Poland, C.L. Ross, The outer solar corona as observed from skylab: preliminary results. Astrophys. J. Lett. 187, 85 (1974). doi: $10.1086 / 181402$

J.T.T. Mäkinen, J.-L. Bertaux, H. Laakso, T. Pulkkinen, T. Summanen, E. Kyrölä, W. Schmidt, E. Quémerais, R. Lallement, Discovery of a comet by its Lyman- $\alpha$ emission [comet C/1997 K2 (SOHO)]. Nature 405, 321-322 (2000)

S. Mancuso, Water production rate of comet C/1997 H2 (SOHO) near perihelion. Astron. Astrophys. 578, 7 (2015). doi:10.1051/0004-6361/201526132

I. Mann, Interstellar dust in the solar system. Annu. Rev. Astron. Astrophys. 48, 173-203 (2010). doi:10.1146/annurev-astro-081309-130846

I. Mann, A. Czechowski, Dust destruction and ion formation in the inner solar system. Astrophys. J. Lett. 621, 73-76 (2005). doi:10.1086/429129

I. Mann, H. Kimura, D.A. Biesecker, B.T. Tsurutani, E. Grün, R.B. McKibben, J.-C. Liou, R.M. MacQueen, T. Mukai, M. Guhathakurta, P. Lamy, Dust near the Sun. Space Sci. Rev. 110, 269-305 (2004). doi:10.1023/B:SPAC.0000023440.82735.ba

C.J. Manser, B.T. Gänsicke, D. Koester, T.R. Marsh, J. Southworth, Another one grinds the dust: variability of the planetary debris disc at the white dwarf SDSS J104341.53+085558.2. Mon. Not. R. Astron. Soc. 462, 1461-1469 (2016). doi:10.1093/mnras/stw1760 
J.N. Marcus, Forward-scattering enhancement of comet brightness. I. Background and model. Int. Comet Quart., 39-66 (2007)

J.N. Marcus, Another unsung Lowell Observatory achievement: the first infrared observation of a comet. ArXiv e-prints (2013a)

J.N. Marcus, Comet C/2012 S1 (Ison). Central Bureau Electronic Telegrams 3723 (2013b)

B.G. Marsden, The sungrazing comet group. Astron. J. 72, 1170-1183 (1967)

B.G. Marsden, The sungrazing comet group. II. Astron. J. 98, 2306-2321 (1989). doi:10.1086/115301

B.G. Marsden, Sungrazing comets. Annu. Rev. Astron. Astrophys. 43, 75-102 (2005)

B.G. Marsden, M. Meyer, Non-Kreutz near-Sun comet groups. Int. Astron. U. Circ. 7832, 1 (2002)

B.G. Marsden, G.V. Williams, Catalogue of Cometary Orbits (IAU Minor Planet Center/Central Bureau for Astronomical Telegrams, Cambridge, 2008)

B.G. Marsden, Z. Sekanina, D.K. Yeomans, Comets and nongravitational forces. V. Astron. J. 78, 211 (1973). doi:10.1086/111402

R. Martín-Doménech, G.M. Muñoz Caro, J. Bueno, F. Goesmann, Thermal desorption of circumstellar and cometary ice analogs. Astron. Astrophys. 564, 8 (2014). doi:10.1051/0004-6361/201322824

M. Masek, J. Jurysek, J. Cerny, M. Jelinek, R. Cunniffe, A.J. Castro-Tirado, Comet C/2015 D1 (SOHO). Central Bureau Electronic Telegrams 4073 (2015)

P.I. McCauley, S.H. Saar, J.C. Raymond, Y.-K. Ko, P. Saint-Hilaire, Extreme-ultraviolet and X-ray observations of comet Lovejoy (C/2011 W3) in the lower corona. Astrophys. J. 768, 161 (2013). doi:10.1088/0004-637X/768/2/161

K.J. Meech, O.R. Hainaut, B.G. Marsden, Comet nucleus size distributions from HST and Keck telescopes. Icarus 170, 463-491 (2004). doi:10.1016/j.icarus.2004.03.014

K.J. Meech, B. Yang, J. Kleyna, M. Ansdell, H.-F. Chiang, O. Hainaut, J.-B. Vincent, H. Boehnhardt, A. Fitzsimmons, T. Rector, T. Riesen, J.V. Keane, B. Reipurth, H.H. Hsieh, P. Michaud, G. Milani, E. Bryssinck, R. Ligustri, R. Trabatti, G.-P. Tozzi, S. Mottola, E. Kuehrt, B. Bhatt, D. Sahu, C. Lisse, L. Denneau, R. Jedicke, E. Magnier, R. Wainscoat, Outgassing behavior of C/2012 S1 (ISON) from 2011 September to 2013 June. Astrophys. J. Lett. 776, 20 (2013). doi:10.1088/2041-8205/776/2/L20

K.J. Meech, B. Yang, J. Kleyna, O.R. Hainaut, S. Berdyugina, J.V. Keane, M. Micheli, A. Morbidelli, R.J. Wainscoat, Inner solar system material discovered in the Oort cloud. Sci. Adv. 2, 1600038 (2016)

G.J. Melnick, D.A. Neufeld, K.E.S. Ford, D.J. Hollenbach, M.L.N. Ashby, Discovery of water vapour around IRC+10216 as evidence for comets orbiting another star. Nature 412, 160-163 (2001)

D.A. Mendis, N.C. Wickramasinghe, Composition of cometary dust: the case against silicates. Astrophys. Space Sci. 37, 13 (1975)

M. Micheli, F. Bernardi, D.J. Tholen, Updated analysis of the dynamical relation between asteroid 2003 EH1 and comets C/1490 Y1 and C/1385 U1. Mon. Not. R. Astron. Soc. 390, 6-8 (2008). doi:10.1111/j.1745-3933.2008.00510.x

S.L. Montgomery, B.Y. Welsh, Detection of variable gaseous absorption features in the debris disks around young A-type stars. Publ. Astron. Soc. Pac. 124, 1042-1056 (2012). doi:10.1086/668293

A. Morbidelli, H. Rickman, Comets as collisional fragments of a primordial planetesimal disk. Astron. Astrophys. 583, 43 (2015). doi:10.1051/0004-6361/201526116

N. Movshovitz, E. Asphaug, D. Korycansky, Numerical modeling of the disruption of comet D/1993 F2 Shoemaker-Levy 9 representing the progenitor by a gravitationally bound assemblage of randomly shaped polyhedra. Astrophys. J. 759, 93 (2012). doi:10.1088/0004-637X/759/2/93

D. Nesvorný, P. Jenniskens, H.F. Levison, W.F. Bottke, D. Vokrouhlický, M. Gounelle, Cometary origin of the zodiacal cloud and carbonaceous micrometeorites. Implications for hot debris disks. Astrophys. J. 713, 816-836 (2010). doi:10.1088/0004-637X/713/2/816

M. Neugebauer, G. Gloeckler, J.T. Gosling, A. Rees, R. Skoug, B.E. Goldstein, T.P. Armstrong, M.R. Combi, T. Mäkinen, D.J. McComas, R. von Steiger, T.H. Zurbuchen, E.J. Smith, J. Geiss, L.J. Lanzerotti, Encounter of the Ulysses Spacecraft with the ion tail of comet MCNaught. Astrophys. J. 667, 1262-1266 (2007). doi:10.1086/521019

M.B. Niedner Jr., J.C. Brandt, Interplanetary gas. XXII-plasma tail disconnection events in cometsevidence for magnetic field line reconnection at interplanetary sector boundaries. Astrophys. J. 223, 655-670 (1978). doi:10.1086/156299

K. Nishioka, Finite lifetime fragment model 2 for synchronic band formation in dust tails of comets. Icarus 134, 24-34 (1998). doi:10.1006/icar.1998.5935

P. Notni, W. Thaenert, The striae in the dust tails of great comets-a comparison to various theories. Astron. Nachr. 309, 133-146 (1988). doi:10.1002/asna.2113090210

K. Ohtsuka, S. Nakano, M. Yoshikawa, On the association among periodic comet 96P/Machholz, Arietids, the Marsden comet group, and the Kracht comet group. Publ. Astron. Soc. Jpn. 55, 321-324 (2003)

E. Öpik, Comet Pereyra 1963 e and its Sun-grazing family. Ir. Astron. J. 6, 159 (1963)

E.J. Öpik, Sun-grazing comets and tidal disruption. Ir. Astron. J. 7, 141-161 (1966) 
C. Opitom, E. Jehin, J. Manfroid, M. Gillon, Comet C/2012 S1 (ISON). Central Bureau Electronic Telegrams 3693 (2013)

L. O’Rourke, D. Bockelée-Morvan, N. Biver, B. Altieri, D. Teyssier, L. Jorda, V. Debout, C. Snodgrass, M. Küppers, M. A'Hearn, T.G. Müller, T. Farnham, Herschel and IRAM-30 m observations of comet C/2012 S1 (ISON) at 4.5 AU from the Sun. Astron. Astrophys. 560, 101 (2013). doi:10.1051/ 0004-6361/201322756

T. Page, Far-UV observations of comet Kohoutek and other targets with the S201 electronographic camera on SKYLAB 4, in Electrography and Astronomical Applications, ed. by G.L. Chincarini, P.J. Griboval, H.J. Smith (1974), pp. 297-305

M. Pajola, J.-B. Vincent, C. Güttler, J.-C. Lee, I. Bertini, M. Massironi, E. Simioni, F. Marzari, L. Giacomini, A. Lucchetti, C. Barbieri, G. Cremonese, G. Naletto, A. Pommerol, M.R. El-Maarry, S. Besse, M. Küppers, F. La Forgia, M. Lazzarin, N. Thomas, A.-T. Auger, H. Sierks, P. Lamy, R. Rodrigo, D. Koschny, H. Rickman, H.U. Keller, J. Agarwal, M.F. A'Hearn, M.A. Barucci, J.-L. Bertaux, V. Da Deppo, B. Davidsson, M. De Cecco, S. Debei, F. Ferri, S. Fornasier, M. Fulle, O. Groussin, P.J. Gutierrez, S.F. Hviid, W.-H. Ip, L. Jorda, J. Knollenberg, J.-R. Kramm, E. Kürt, L.M. Lara, Z.-Y. Lin, J.J. Lopez Moreno, S. Magrin, S. Marchi, H. Michalik, R. Moissl, S. Mottola, N. Oklay, F. Preusker, F. Scholten, C. Tubiana, Size-frequency distribution of boulders $>=7 \mathrm{~m}$ on comet 67P/Churyumov-Gerasimenko. Astron. Astrophys. 583, 37 (2015). doi:10.1051/0004-6361/201525975

J.M. Pasachoff, V. Rušin, M. Druckmüller, P. Aniol, M. Saniga, M. Minarovjech, The 2008 August 1 eclipse solar-minimum corona unraveled. Astrophys. J. 702, 1297-1308 (2009). doi:10.1088/ 0004-637X/702/2/1297

M. Pätzold, T. Andert, M. Hahn, S.W. Asmar, J.-P. Barriot, M.K. Bird, B. Häusler, K. Peter, S. Tellmann, E. Grün, P.R. Weissman, H. Sierks, L. Jorda, R. Gaskell, F. Preusker, F. Scholten, A homogeneous nucleus for comet 67P/Churyumov-Gerasimenko from its gravity field. Nature 530, 63-65 (2016). doi:10.1038/nature 16535

C.D. Perrine, The search for an intra-mercurial planet at the total solar eclipse of 1901, May 18. Publ. Astron. Soc. Pac. 14, 160 (1902). doi:10.1086/121490

W.D. Pesnell, P. Bryans, The time-dependent chemistry of cometary debris in the solar corona. Astrophys. J. 785, 50 (2014). doi:10.1088/0004-637X/785/1/50

J. Pittichová, Z. Sekanina, K. Birkle, H. Boehnhardt, D. Engels, P. Keller, An early investigation of the striated tail of comet Hale-Bopp (C/1995 O1). Earth Moon Planets 78, 329-338 (1997). doi:10.1023/A:1006242209416

M.S. Povich, J.C. Raymond, G.H. Jones, M. Uzzo, Y.-K. Ko, P.D. Feldman, P.L. Smith, B.G. Marsden, T.N. Woods, Doubly ionized carbon observed in the plasma tail of comet Kudo-Fujikawa. Science 302, 1949-1952 (2003)

G.W. Preston, The spectrum of Ikeya-Seki (1965f). Astrophys. J. 147, 718-742 (1967)

Y. Ramanjooloo, Comets as natural laboratories: interpretations of the structure of the inner heliosphere, $\mathrm{PhD}$ thesis, UCL, University College London, Gower Street, London WC1E 6BT, UK (2015). http://discovery.ucl.ac.uk/1471003/

Y. Ramanjooloo, G.H. Jones, A.J. Coates, M.J. Owens, K. Battams, Comparing solar wind velocity measurements derived from Sun-grazing comet Lovejoy (C/2011 W3) with solar wind models. AGU Fall Meeting Abstracts (2012)

J.C. Raymond, S. Fineschi, P.L. Smith, L. Gardner, R. O’Neal, A. Ciaravella, J.L. Kohl, B. Marsden, G.V. Williams, C. Benna, S. Giordano, G. Noci, D. Jewitt, Solar wind at 6.8 solar radii from UVCS observation of comet C/1996 Y1. Astrophys. J. 508, 410-417 (1998). doi:10.1086/306391

J.C. Raymond, M. Uzzo, Y.-K. Ko, S. Mancuso, R. Wu, L. Gardner, J.L. Kohl, B. Marsden, P.L. Smith, Far-ultraviolet observations of comet 2P/Encke at perihelion. Astrophys. J. 564, 1054-1060 (2002). doi: $10.1086 / 324545$

J.C. Raymond, P.I. McCauley, S.R. Cranmer, C. Downs, The solar corona as probed by comet Lovejoy (C/2011 W3). Astrophys. J. 788, 152 (2014). doi:10.1088/0004-637X/788/2/152

J.C. Raymond et al., Comet C/2011 W3 (Lovejoy) between 2 and 10 Solar Radii: physical parameters of the comet and the corona. Astrophys. J. (2017). Submitted

W.T. Reach, J. Vaubaillon, M.S. Kelley, C.M. Lisse, M.V. Sykes, Distribution and properties of fragments and debris from the split comet 73P/Schwassmann-Wachmann 3 as revealed by Spitzer Space Telescope. Icarus 203, 571-588 (2009). doi:10.1016/j.icarus.2009.05.027

J.E. Richardson, H.J. Melosh, C.M. Lisse, B. Carcich, A ballistics analysis of the deep impact ejecta plume: determining comet Tempel 1's gravity, mass, and density. Icarus 190, 357-390 (2007). doi:10.1016/ j.icarus.2007.08.001

H. Rickman, C. Froeschle, Cometary dynamics. Celest. Mech. 43, 243-263 (1988)

S.D. Rodgers, S.B. Charnley, W.F. Huebner, D.C. Boice, in Physical Processes and Chemical Reactions in Cometary Comae, ed. by M.C. Festou, H.U. Keller, H.A. Weaver (2004), pp. 505-522 
N.H. Samarasinha, B.E.A. Mueller, Relating changes in cometary rotation to activity: current status and applications to comet C/2012 S1 (ISON). Astrophys. J. Lett. 775, 5 (2013). doi:10.1088/ 2041-8205/775/1/L10

P. Santos-Sanz, J.L. Ortiz, N. Morales, R. Duffard, F. Pozuelos, F. Moreno, E. Fernández-Valenzuela, Shortterm variability of comet C/2012 S1 (ISON) at 4.8 AU from the Sun. Astron. Astrophys. 575, 52 (2015). doi:10.1051/0004-6361/201425265

D.G. Schleicher, R.L. Millis, P.V. Birch, Narrowband photometry of comet P/Halley: variation with heliocentric distance, season, and solar phase angle. Icarus 132, 397-417 (1998). doi:10.1006/icar.1997.5902

H.E. Schlichting, C.I. Fuentes, D.E. Trilling, Initial planetesimal sizes and the size distribution of small Kuiper belt objects. Astron. J. 146, 36 (2013). doi:10.1088/0004-6256/146/2/36

C.A. Schmidt, R.E. Johnson, J. Baumgardner, M. Mendillo, Observations of sodium in the coma of comet C/2012 S1 (ISON) during outburst. Icarus 247, 313-318 (2015). doi:10.1016/j.icarus.2014.10.022

C.J. Schrijver, J.C. Brown, K. Battams, P. Saint-Hilaire, W. Liu, H. Hudson, W.D. Pesnell, Destruction of Sun-grazing comet C/2011 N3 (SOHO) within the low solar corona. Science 335, 324-328 (2012). doi: $10.1126 /$ science. 1211688

R. Schulz, M. Hilchenbach, Y. Langevin, J. Kissel, J. Silen, C. Briois, C. Engrand, K. Hornung, D. Baklouti, A. Bardyn, H. Cottin, H. Fischer, N. Fray, M. Godard, H. Lehto, L. Le Roy, S. Merouane, F.-R. OrthousDaunay, J. Paquette, J. Rynö, S. Siljeström, O. Stenzel, L. Thirkell, K. Varmuza, B. Zaprudin, Comet 67P/Churyumov-Gerasimenko sheds dust coat accumulated over the past four years. Nature 518, 216218 (2015). doi:10.1038/nature 14159

G. Schumacher, J. Gay, An attempt to detect vulcanoids with SOHO/LASCO images. I. Scale relativity and quantization of the solar system. Astron. Astrophys. 368, 1108-1114 (2001). doi:10.1051/ 0004-6361:20000356

D.B. Seaton, D. Berghmans, B. Nicula, J.-P. Halain, A. De Groof, T. Thibert, D.S. Bloomfield, C.L. Raftery, P.T. Gallagher, F. Auchère, J.-M. Defise, E. D’Huys, J.-H. Lecat, E. Mazy, P. Rochus, L. Rossi, U. Schühle, V. Slemzin, M.S. Yalim, J. Zender, The SWAP EUV imaging telescope part I: instrument overview and pre-flight testing. Sol. Phys. 286, 43-65 (2013). doi:10.1007/s11207-012-0114-6

Z. Sekanina, On the origin of the Kreutz family of Sun-grazing comets. Bull. Astron. Inst. Czechoslov. 18, 198-199 (1967)

Z. Sekanina, The path and surviving tail of a comet that fell into the Sun. Astron. J. 87, 1059-1072 (1982a). doi:10.1086/113190

Z. Sekanina, The problem of split comets in review, in IAU Colloq. 61: Comet Discoveries, Statistics, and Observational Selection, ed. by L.L. Wilkening (1982b), pp. 251-287

Z. Sekanina, Secondary fragmentation of the solar and heliospheric observatory sungrazing comets at very large heliocentric distance. Astrophys. J. Lett. 542, 147-150 (2000). doi:10.1086/312943

Z. Sekanina, Runaway fragmentation of sungrazing comets observed with the solar and heliospheric observatory. Astrophys. J. 576, 1085-1089 (2002a). doi:10.1086/341801

Z. Sekanina, Statistical investigation and modeling of sungrazing comets discovered with the solar and heliospheric observatory. Astrophys. J. 566, 577-598 (2002b). doi:10.1086/324335

Z. Sekanina, Erosion model for the sungrazing comets observed with the solar and heliospheric observatory. Astrophys. J. 597, 1237-1265 (2003). doi:10.1086/378192

Z. Sekanina, P.W. Chodas, Common origin of two major sungrazing comets. Astrophys. J. 581, 760-769 (2002a). doi:10.1086/344216

Z. Sekanina, P.W. Chodas, Fragmentation origin of major sungrazing comets C/1970 K1, C/1880 C1, and C/1843 D1. Astrophys. J. 581, 1389-1398 (2002b). doi:10.1086/344261

Z. Sekanina, P.W. Chodas, Fragmentation hierarchy of bright sungrazing comets and the birth and orbital evolution of the Kreutz system. I. Two-superfragment model. Astrophys. J. 607, 620-639 (2004). doi: $10.1086 / 383466$

Z. Sekanina, P.W. Chodas, Origin of the Marsden and Kracht groups of sunskirting comets. I. Association with comet 96P/Machholz and its interplanetary complex. Astrophys. J. Suppl. Ser. 161, 551-586 (2005). doi:10.1086/497374

Z. Sekanina, P.W. Chodas, Fragmentation hierarchy of bright sungrazing comets and the birth and orbital evolution of the Kreutz system. II. The case for cascading fragmentation. Astrophys. J. 663, 657-676 (2007). doi:10.1086/517490

Z. Sekanina, P.W. Chodas, A new orbit determination for bright sungrazing comet of 1843. Astrophys. J. 687, 1415-1422 (2008)

Z. Sekanina, P.W. Chodas, Comet C/2011 W3 (Lovejoy): orbit determination, outbursts, disintegration of nucleus, dust-tail morphology, and relationship to new cluster of bright sungrazers. Astrophys. J. 757, 127 (2012). doi:10.1088/0004-637X/757/2/127

Z. Sekanina, J.A. Farrell, The striated dust tail of comet West 1976 VI as a particle fragmentation phenomenon. Astron. J. 85, 1538-1554 (1980). doi:10.1086/112831 
Z. Sekanina, R. Kracht, Disintegration of comet C/2012 S1 (ISON) shortly before perihelion: evidence from independent data sets. ArXiv e-prints (2014)

Z. Sekanina, R. Kracht, Strong erosion-driven nongravitational effects in orbital motions of the Kreutz sungrazing system's dwarf comets. Astrophys. J. 801, 135 (2015). doi:10.1088/0004-637X/801/2/135

Z. Sekanina, D.K. Yeomans, Orbital motion, nucleus precession, and splitting of periodic comet Brooks 2. Astron. J. 90, 2335-2352 (1985). doi:10.1086/113939

Z. Sekenina, J. Pittichová, Distribution law for particle fragmentation times in a theory for striated tails of dust comets: application to comet Hale-Bopp (C/1995 O1). Earth Moon Planets 78, 339-346 (1997). doi:10.1023/A:1006294226254

N.R. Sheeley Jr., R.A. Howard, M.J. Koomen, D.J. Michels, Coronagraphic observations of two new sungrazing comets. Nature 300, 239-242 (1982)

Y. Shinnaka, H. Kawakita, H. Kobayashi, M. Nagashima, D.C. Boice, ${ }^{14} \mathrm{NH}_{2} /{ }^{15} \mathrm{NH}_{2}$ ratio in comet C/2012 S1 (ISON) observed during its outburst in 2013 November. Astrophys. J. Lett. 782, 16 (2014). doi:10.1088/2041-8205/782/2/L16

Y. Shou, M. Combi, Y.-D. Jia, T. Gombosi, G. Toth, M. Rubin, The plasma environment in comets over a wide range of heliocentric distances: application to comet C/2006 P1 (McNaught). Astrophys. J. 809, 156 (2015). doi:10.1088/0004-637X/809/2/156

H. Sierks, C. Barbieri, P.L. Lamy, R. Rodrigo, D. Koschny, H. Rickman, H.U. Keller, J. Agarwal, M.F. A'Hearn, F. Angrilli, A.-T. Auger, M.A. Barucci, J.-L. Bertaux, I. Bertini, S. Besse, D. Bodewits, C. Capanna, G. Cremonese, V. Da Deppo, B. Davidsson, S. Debei, M. De Cecco, F. Ferri, S. Fornasier, M. Fulle, R. Gaskell, L. Giacomini, O. Groussin, P. Gutierrez-Marques, P.J. Gutiérrez, C. Güttler, N. Hoekzema, S.F. Hviid, W.-H. Ip, L. Jorda, J. Knollenberg, G. Kovacs, J.R. Kramm, E. Kührt, M. Küppers, F. La Forgia, L.M. Lara, M. Lazzarin, C. Leyrat, J.J. Lopez Moreno, S. Magrin, S. Marchi, F. Marzari, M. Massironi, H. Michalik, R. Moissl, S. Mottola, G. Naletto, N. Oklay, M. Pajola, M. Pertile, F. Preusker, L. Sabau, F. Scholten, C. Snodgrass, N. Thomas, C. Tubiana, J.-B. Vincent, K.-P. Wenzel, M. Zaccariotto, M. Pätzold, On the nucleus structure and activity of comet $67 \mathrm{P} / \mathrm{Churyumov-}$ Gerasimenko. Science 347, 1044 (2015). doi:10.1126/science.aaa1044

C.D. Slaughter, The emission spectrum of comet Ikeya-Seki 1965-f at perihelion passage. Astron. J. 74, 929-943 (1969)

C. Snodgrass, S.C. Lowry, A. Fitzsimmons, Photometry of cometary nuclei: rotation rates, colours and a comparison with Kuiper belt objects. Mon. Not. R. Astron. Soc. 373, 1590-1602 (2006). doi:10.1111/j.1365-2966.2006.11121.x

C. Snodgrass, A. Fitzsimmons, S.C. Lowry, P. Weissman, The size distribution of Jupiter family comet nuclei. Mon. Not. R. Astron. Soc. 414, 458-469 (2011). doi:10.1111/j.1365-2966.2011.18406.x

T. Spohn, J. Knollenberg, A.J. Ball, M. Banaszkiewicz, J. Benkhoff, M. Grott, J. Grygorczuk, C. Hüttig, A. Hagermann, G. Kargl, E. Kaufmann, N. Kömle, E. Kührt, K.J. Kossacki, W. Marczewski, I. Pelivan, R. Schrödter, K. Seiferlin, Thermal and mechanical properties of the near-surface layers of comet 67P/Churyumov-Gerasimenko. Science 349(2) (2015). doi:10.1126/science.aab0464

O.C. St. Cyr, R.C. Altrock, Groundbased observations of the Sun's corona following the perihelia of sungrazing comets, in Bulletin of the American Astronomical Society. Bulletin of the American Astronomical Society, vol. 25 (1993), p. 1303

J.K. Steckloff, S.A. Jacobson, The formation of striae within cometary dust tails by a sublimation-driven YORP-like effect. Icarus 264, 160-171 (2016). doi:10.1016/j.icarus.2015.09.021

J.K. Steckloff, B.C. Johnson, T. Bowling, H. Jay Melosh, D. Minton, C.M. Lisse, K. Battams, Dynamic sublimation pressure and the catastrophic breakup of comet ISON. Icarus 258, 430-437 (2015a). doi:10.1016/j.icarus.2015.06.032

J.K. Steckloff, J. Keane, M.M. Knight, J. Kleyna, S.N. Milam, I. Coulson, K. Meech, S.A. Jacobson, H.J. Melosh, The size and fragmentation of the nucleus of comet C/2012 S1 (ISON), in Lunar and Planetary Science Conference. Lunar and Planetary Science Conference, vol. 46 (2015b), p. 2723

A.J. Steffl, N.J. Cunningham, A.B. Shinn, D.D. Durda, S.A. Stern, A search for vulcanoids with the STEREO heliospheric imager. Icarus 223, 48-56 (2013). doi:10.1016/j.icarus.2012.11.031

R. Strom, Daytime observations of sungrazing comets in Chinese annals. Astron. Astrophys. 387, 17-20 (2002). doi:10.1051/0004-6361:20020496

H. Su, T. Hoffman, M. Kusiak, R. Kracht, K. Battams, B.G. Marsden, Comets C/2008 N6, 2008 N7, 2008 O4, 2008 R7 (SOHO). Minor Planet Electronic Circulars 2008 (2008)

M.B. Swift, G.F. Mitchell, Models of the cometary coma in which abundances are calculated for various heliocentric distances. Icarus 47, 412-430 (1981). doi:10.1016/0019-1035(81)90189-5

K. Szegö, K.-H. Glassmeier, R. Bingham, A. Bogdanov, C. Fischer, G. Haerendel, A. Brinca, T. Cravens, E. Dubinin, K. Sauer, L. Fisk, T. Gombosi, N. Schwadron, P. Isenberg, M. Lee, C. Mazelle, E. Möbius, U. Motschmann, V.D. Shapiro, B. Tsurutani, G. Zank, Physics of mass loaded plasmas. Space Sci. Rev. 94, 429-671 (2000) 
G. Tancredi, J.A. Fernández, H. Rickman, J. Licandro, Nuclear magnitudes and the size distribution of Jupiter family comets. Icarus 182, 527-549 (2006). doi:10.1016/j.icarus.2006.01.007

A.D. Thackeray, M.W. Feast, B. Warner, Daytime spectra of comet Ikeya-Seki near perihelion. Astrophys. J. 143, 276 (1966). doi:10.1086/148506

I. Toth, C.M. Lisse, On the rotational breakup of cometary nuclei and centaurs. Icarus 181, 162-177 (2006). doi:10.1016/j.icarus.2005.10.012

M. Uchina, K. Battams, G.V. Williams, Comet C/2011 n3 (SOHO). Minor Planet Electronic Circulars 2011 (2011)

M. Uzzo, J.C. Raymond, D. Biesecker, B. Marsden, C. Wood, Y.-K. Ko, R. Wu, Results from UVCS and LASCO observation of the sungrazing comet C/2000 C6. Astrophys. J. 558, $403-410$ (2001). doi:10.1086/322473

D. Veras, B.T. Gänsicke, Detectable close-in planets around white dwarfs through late unpacking. Mon. Not. R. Astron. Soc. 447, 1049-1058 (2015). doi:10.1093/mnras/stu2475

D. Veras, S.A. Jacobson, B.T. Gänsicke, Post-main-sequence debris from rotation-induced YORP break-up of small bodies. Mon. Not. R. Astron. Soc. 445, 2794-2799 (2014). doi:10.1093/mnras/stu1926

D. Veras, A.J. Mustill, B.T. Gaensicke, The unstable fate of the planet orbiting the A-star in the HD 131399 triple stellar system. ArXiv e-prints (2016)

J.-B. Vincent, M.F. A’Hearn, Z.-Y. Lin, M.R. El-Maarry, M. Pajola, H. Sierks, C. Barbieri, P.L. Lamy, R. Rodrigo, D. Koschny, H. Rickman, H.U. Keller, J. Agarwal, M.A. Barucci, J.-L. Bertaux, I. Bertini, S. Besse, D. Bodewits, G. Cremonese, V. Da Deppo, B. Davidsson, S. Debei, M. De Cecco, J. Deller, S. Fornasier, M. Fulle, A. Gicquel, O. Groussin, P.J. Gutiérrez, P. Gutiérrez-Marquez, C. Güttler, S. Höfner, M. Hofmann, S.F. Hviid, W.-H. Ip, L. Jorda, J. Knollenberg, G. Kovacs, J.-R. Kramm, E. Kührt, M. Küppers, L.M. Lara, M. Lazzarin, J.J. Lopez Moreno, F. Marzari, M. Massironi, S. Mottola, G. Naletto, N. Oklay, F. Preusker, F. Scholten, X. Shi, N. Thomas, I. Toth, C. Tubiana, Summer fireworks on comet 67P. Mon. Not. R. Astron. Soc. 462, 184-194 (2016). doi:10.1093/mnras/stw2409

D. Vokrouhlický, P. Farinella, W.F. Bottke, The depletion of the putative vulcanoid population via the Yarkovsky effect. Icarus 148, 147-152 (2000). doi:10.1006/icar.2000.6468

A. Vourlidas, C.J. Davis, C.J. Eyles, S.R. Crothers, R.A. Harrison, R.A. Howard, J.D. Moses, D.G. Socker, First direct observation of the interaction between a comet and a coronal mass ejection leading to a complete plasma tail disconnection. Astrophys. J. 668, 79-82 (2007). doi:10.1086/522587

A. Vourlidas, R.A. Howard, S.P. Plunkett, C.M. Korendyke, A.F.R. Thernisien, D. Wang, N. Rich, M.T. Carter, D.H. Chua, D.G. Socker, M.G. Linton, J.S. Morrill, S. Lynch, A. Thurn, P. Van Duyne, R. Hagood, G. Clifford, P.J. Grey, M. Velli, P.C. Liewer, J.R. Hall, E.M. DeJong, Z. Mikic, P. Rochus, E. Mazy, V. Bothmer, J. Rodmann, The Wide-Field Imager for Solar Probe Plus (WISPR). Space Sci. Rev. 204, 83-130 (2016). doi:10.1007/s11214-014-0114-y

K. Watson, B.C. Murray, H. Brown, The stability of volatiles in the solar system. Icarus 1, 317-327 (1963). doi:10.1016/0019-1035(62)90030-1

H.A. Weaver, P.D. Feldman, M.F. A'Hearn, C. Arpigny, The activity and size of the nucleus of comet HaleBopp (C/1995 O1). Science 275, 1900-1904 (1997). doi:10.1126/science.275.5308.1900

H.A. Weaver, Z. Sekanina, I. Toth, C.E. Delahodde, O.R. Hainaut, P.L. Lamy, J.M. Bauer, M.F. A'Hearn, C. Arpigny, M.R. Combi, J.K. Davies, P.D. Feldman, M.C. Festou, R. Hook, L. Jorda, M.S.W. Keesey, C.M. Lisse, B.G. Marsden, K.J. Meech, G.P. Tozzi, R. West, HST and VLT investigations of the fragments of comet C/1999 S4 (LINEAR). Science 292, 1329-1334 (2001). doi:10.1126/science.1058606

H.A. Weaver, C.M. Lisse, M.J. Mutchler, P. Lamy, I. Toth, W.T. Reach, Hubble space telescope investigation of the disintegration of 73P/Schwassmann-Wachmann 3, in Bulletin of the American Astronomical Society. Bulletin of the American Astronomical Society, vol. 38 (2006), p. 490

R. Wegmann, MHD model calculations for the effect of interplanetary shocks on the plasma tail of a comet. Astron. Astrophys. 294, 601-614 (1995)

R. Wegmann, The effect of some solar wind disturbances on the plasma tail of a comet: models and observations. Astron. Astrophys. 358, 759-775 (2000)

R. Wegmann, Large-scale disturbance of the solar wind by a comet. Astron. Astrophys. 389, 1039-1046 (2002). doi:10.1051/0004-6361:20020595

R. Wegmann, K. Dennerl, X-ray tomography of a cometary bow shock. Astron. Astrophys. 430, 33-36 (2005). doi:10.1051/0004-6361:200400124

R. Wegmann, K. Dennerl, C.M. Lisse, The morphology of cometary X-ray emission. Astron. Astrophys. 428, 647-661 (2004). doi:10.1051/0004-6361:20041008

R. Wegmann, H.U. Schmidt, W.F. Huebner, D.C. Boice, Cometary MHD and chemistry. Astron. Astrophys. 187, 339-350 (1987)

R. Wegmann, H.U. Schmidt, C.M. Lisse, K. Dennerl, J. Englhauser, X-rays from comets generated by energetic solar wind particles. Planet. Space Sci. 46, 603-612 (1998). doi:10.1016/S0032-0633(97)00196-7 
P.R. Weissman, Nongravitational perturbations of long-period comets. Astron. J. 84, 580-584 (1979). doi: $10.1086 / 112453$

P.R. Weissman, Physical loss of long-period comets. Astron. Astrophys. 85, 191-196 (1980)

P.R. Weissman, Cometary impacts with the Sun—physical and dynamical considerations. Icarus 55, 448-454 (1983). doi:10.1016/0019-1035(83)90115-X

P.R. Weissman, Are cometary nuclei primordial rubble piles? Nature 320, 242-244 (1986). doi:10.1038/ $320242 \mathrm{a} 0$

P.R. Weissman, H.H. Kieffer, Thermal modeling of cometary nuclei. Icarus 47, 302-311 (1981). doi:10.1016/ 0019-1035(81)90177-9

P.R. Weissman, H.F. Levison, Origin and evolution of the unusual object 1996 PW: asteroids from the Oort cloud? Astrophys. J. Lett. 488, 133-136 (1997). doi:10.1086/310940

P.R. Weissman, S.C. Lowry, The size distribution of Jupiter-family cometary nuclei, in Lunar and Planetary Science Conference, ed. by S. Mackwell, E. Stansbery Lunar and Planetary Science Conference, vol. 34 (2003)

P.R. Weissman, E. Asphaug, S.C. Lowry, Structure and density of cometary nuclei, in Comets II, ed. by M.C. Festou, H.U. Keller, H.A. Weaver (University of Arizona Press, Tucson, 2004), pp. 337-357

P.R. Weissman, E. Asphaug, N. Movshovitz, E. Rosenberg, Dynamical simulations of the tidal disruption of Kreutz-group sungrazing comets, in AAS/Division for Planetary Sciences Meeting Abstracts. AAS/Division for Planetary Sciences Meeting Abstracts, vol. 44 (2012), p. 514

B.Y. Welsh, S. Montgomery, Circumstellar gas-disk variability around A-type stars: the detection of exocomets? Publ. Astron. Soc. Pac. 125, 759-774 (2013). doi:10.1086/671757

F.L. Whipple, 1983 TB and the Geminid meteors. Int. Astron. U. Circ. 3881 (1983)

K. Wilhelm, W. Curdt, E. Marsch, U. Schühle, P. Lemaire, A. Gabriel, J.-C. Vial, M. Grewing, M.C.E. Huber, S.D. Jordan, A.I. Poland, R.J. Thomas, M. Kühne, J.G. Timothy, D.M. Hassler, O.H.W. Siegmund, SUMER - solar ultraviolet measurements of emitted radiation. Sol. Phys. 162, 189-231 (1995). doi:10.1007/BF00733430

G.V. Williams, Comet C/2015 D1 (SOHO). Central Bureau Electronic Telegrams 4067 (2015)

L.L. Williams, G.P. Zank, Effect of magnetic field geometry on the wave signature of the pickup of interstellar neutrals. J. Geophys. Res. 99, 19 (1994). doi:10.1029/94JA01657

D.H. Wooden, C.E. Woodward, D.E. Harker, M.S. Kelley, M. Sitko, W.T. Reach, I. De Pater, R.D. Gehrz, L. Kolokolova, A.L. Cochran, A.J. McKay, K. Reardon, G. Cauzzi, G. Tozzi, D.J. Christian, D.B. Jess, M. Mathioudakis, C.M. Lisse, J.P. Morgenthaler, M.M. Knight, Comet C/2012 S1 (ISON): observations of the dust grains from SOFIA and of the atomic gas from NSO Dunn and McMath-Pierce solar telescopes (invited). AGU Fall Meeting Abstracts (2013)

J.-P. Wuelser, J.R. Lemen, T.D. Tarbell, C.J. Wolfson, J.C. Cannon, B.A. Carpenter, D.W. Duncan, G.S. Gradwohl, S.B. Meyer, A.S. Moore, R.L. Navarro, J.D. Pearson, G.R. Rossi, L.A. Springer, R.A. Howard, J.D. Moses, J.S. Newmark, J.-P. Delaboudiniere, G.E. Artzner, F. Auchere, M. Bougnet, P. Bouyries, F. Bridou, J.-Y. Clotaire, G. Colas, F. Delmotte, A. Jerome, M. Lamare, R. Mercier, M. Mullot, M.-F. Ravet, X. Song, V. Bothmer, W. Deutsch, EUVI: the STEREO-SECCHI extreme ultraviolet imager, in Telescopes and Instrumentation for Solar Astrophysics, ed. by S. Fineschi, M.A. Gummin Proc. of SPIE, vol. 5171 (2004), pp. 111-122. doi:10.1117/12.506877

Q.-Z. Ye, M.-T. Hui, R. Kracht, P.A. Wiegert, Where are the mini Kreutz-family comets? Astrophys. J. 796, 83 (2014). doi:10.1088/0004-637X/796/2/83 